\title{
Bargaining, Reputation and Equilibrium Selection in Repeated Games with Contracts ${ }^{1}$
}

\author{
Dilip Abreu and David Pearce ${ }^{2}$
}

December 6, 2006

\footnotetext{
${ }^{1}$ We would like to thank Ennio Stacchetti for his help, and seminar participants at numerous universities for their comments. We are grateful to Eddie Dekel and the anonymous referees, who, in addition to making many helpful suggestions, were instrumental in shifting the focus of this paper from repeated games without contracts, to the more tractable environment in which legally enforceable contracts are available. This research was supported by NSF grant SES-0417846 and by NYU, Princeton University and Yale University.

${ }^{2}$ Princeton University and NYU, respectively.
} 


\begin{abstract}
Consider a two-person intertemporal bargaining problem in which players choose actions and offers each period and collect payoffs (as function of that period's actions) while bargaining proceeds. This can alternatively be viewed as an infinitely-repeated game wherein players can offer one another enforceable contracts that govern play for the rest of the game. Theory is silent regarding how the surplus is likely to be split, because a folk theorem applies. Perturbing such a game with a rich set of behavioral types for each player yields a specific asymptotic prediction for how the surplus will be divided, as the perturbation probabilities approach zero. Behavioral types may follow nonstationary strategies and respond to the opponent's play. In equilibrium, rational players initally choose a behavioral type to imitate, and a war of attrition ensues. How much should a player try to get, and how should she behave while waiting for the resolution of bargaining? In both respects she should build her strategy around the advice given by the "Nash bargaining with threats" (NBWT) theory developed for twostage games. In any perfect Bayesian equilibrium, she can guarantee herself virtually her NBWT payoff by imitating a behavioral type with the following simple strategy: in every period, ask for (and accept nothing less than) that player's NBWT share and, while waiting for the other side to concede, take the action Nash recommends as a threat in his two-stage game. The results suggest that there are forces at work in some dynamic games that favor certain payoffs over all others. This is in stark contrast to the classic folk theorems, to the further folk theorems established for repeated games with two-sided reputational perturbations, and to the permissive results obtained in the literature on bargaining with payoffs-as-you-go.
\end{abstract}




\section{Introduction}

What kind of reputation should a bargainer try to establish? Should she claim that her demand will never change, or that she will become more aggressive over time? Should improvements in her opponent's offer be punished as signs of weakness or should she promise to reward them with a softening of her own position? Is it useful to announce deadlines after which offers will be withdrawn? This paper addresses these questions in an essentially full-information two-person bargaining model in which there is a small possibility that each player might be one of a rich variety of behavioral types. For example, to use the terminology of Myerson (1991), rather than optimizing as a fully rational player would, the player might use an " $r$-insistent strategy" that always demands the amount $r$ and never accepts anything less. But the player might instead employ a complex history-dependent strategy, a possibility not considered by previous papers in the behavioral bargaining literature. ${ }^{1}$

Now think about broader bargaining problems in which the players interact in payoff-significant ways before an agreement is reached. Such considerations were introduced by Fernandez and Glazer (1991) and Haller and Holden (1990). ${ }^{2}$ For example, before two countries sign a treaty on trade or pollution abatement, their unilateral policies affect one another's payoffs. Here, possibilities for strategic posturing are even more interesting. Does each party maximize its immediate payoff before agreement, or is some degree of cooperation possible during negotiations? As time passes without agreement, do players treat one another more harshly? Is a player's behavior related to her demand, and to the opponent's demand?

Since our framework will generalize the model of Abreu and Gul (2000)

\footnotetext{
${ }^{1}$ Adopting the idea of introducing behavioral perturbations from Kreps, Milgrom, Roberts and Wilson (1982), Myerson (1991) studied a two-person bargaining game with one-sided uncertainty, one-sided offers and a single type. Abreu and Gul (2000) performed a two-sided analysis with multiple types that we will summarize below, prompting Kambe (1999) to do a limit analysis of a related model as the probabilities of perturbation probabilities approach zero. Working with a model with a single behavioral type on either side, Kornhauser, Rubinstein and Wolinsky (1989) take perturbation probabilities to zero to select one equilibrium in a war of attrition game. Investigating the role of outside options in a model which builds upon Abreu and Gul(2000), Compte and Jehiel (2002) also take perturbation probabilities to zero.

${ }^{2}$ These papers show that even in an alternating-offers bargaining game with symmetric information, it is possible to have a multitude of subgame perfect equilibria, including many with substantial delay to agreement. This class of models is now known as "bargaining with payoffs-as-you-go", and has been studied in much greater generality by Busch and Wen (1995).
} 
in two ways, we pause now to summarize their work. An exogenous protocol specifies the times at which each of two impatient bargainers can make offers about how a fixed surplus will be divided. When an offer is made, the other party can accept (and the proposed division is implemented) or reject (and the bargaining continues). Payoffs of rational players are common knowledge, but for each player $i$, there are exogenous initial probabilities $\pi_{i}(k)>0$ that player $i$ is a $k$-insistent type who will never settle for any amount less than $k$. At the start of play normal players mimic behavioral types. Following the initial choice of types, in the limit as one looks at bargaining protocols allowing more and more frequent offers, a war of attrition ensues in which players either simply stick with their initial demands or concede to their opponent's. Equilibrium outcomes are essentially unique and do not depend on the fine details of the protocol. The way the surplus is divided, and the delay to agreement, depend on the set of behavioral types available for each player to imitate and their initial probabilities, and the discount factors of the players. If initial probabilities that players are behavioral are sufficiently low, there is usually almost no delay to agreement. In the limit as the $\pi_{i}(k)$ 's approach zero, each player's expected payoff coincides with the payoff she would get if the Nash bargaining solution (Nash (1950)) were used to divide the surplus (with disagreement point zero). Kambe (1999) was the first to obtain this kind of Nash bargaining result, in his modification of the Abreu and Gul model.

Our paper considers two impatient players who are bargaining over the surplus generated by the "component game" $G$ that they play in each period. After any history of play and of offers that have been made, the players have the option of entering into an enforceable Pareto-efficient agreement governing play of both parties from that time on. There is some chance that either bargainer might be a behavioral player drawn from a rich finite set of behavioral types. Each of those types plays a particular dynamic strategy in the bargaining game. Its actions and demands might vary over time, and might respond in complicated ways to what the other side offers and does. Both the complexity of behaviors allowed in the sets of types and the fact that a game is played while bargaining proceeds make this a much more complicated model than that of Abreu and Gul.

We obtain strong characterizations of equilibria in the limit analysis as the probabilities of behavioral types approach zero. In particular, the "Nash bargaining with threats" concept (Nash (1953)) describes the equilibrium behavior and expected payoffs in a manner analogous to how the simpler Nash bargaining solution describes the asymptotic equilibria in Kambe (1999) and Abreu and Gul (2000). Thus, perturbing the full-information, play-as-you- 
bargain game with the slight possibility of behavioral types replaces a vast multiplicity of equilibria with a strong prediction about outcomes. This strong prediction is more striking when one views the model as a repeated game in which players can sign binding contracts. ${ }^{3}$ When those contracts are unavailable, the problem of multiple sustainable expectations about future play is so powerful that folk theorems persist even in the face of reputational perturbations (see Chan (2000) and the discussion below). The contractual option provides enough stability to allow reputational perturbations to resolve the issue of how surplus is divided.

Section 2 introduces the model. Section 3 establishes the result for the special case of stationary postures. In Section 4 we provide the general characterization result. Section 5 establishes existence of equilibrium and Section 6 concludes.

\section{Further Related Literature}

The study of "reputation effects" in repeated games originates in three celebrated papers: Kreps, Milgrom, Roberts and Wilson (1982), Kreps and Wilson (1982) and Milgrom and Roberts (1982). A decisive paper by Fudenberg and Levine (1989) showed that a sufficiently patient long-run player facing a series of uninformed short-run players can achieve approximately his Stackelberg payoff or better, for any prior distribution over types he might be that puts positive weight on his Stackelberg type. Analogously, we assume a positive probability that each of the players may be the "Nash bargaining with threats" type.

When both players are infinitely lived, even if player 1 is much more patient than 2, the lower bounds available for 1's perfect equilibrium payoffs are much weaker than those provided by Fudenberg and Levine (see especially Schmidt (1993) and Cripps, Schmidt and Thomas (1996)). One difficulty for an informed player 1 is his lack of "transparency": player 2 cannot tell what type she is facing, and therefore may be unwilling to risk playing a myopic best response to the informed player's Stackelberg action, for fear that he is a vindictive type who will then switch to an action that minimaxes her, for example. ${ }^{4}$ Two papers get around this problem and obtain strong reputa-

\footnotetext{
${ }^{3}$ The ability to make offers also affords the players a communication channel. Therefore, this paper is not a contribution to the literature started by Aumann and Sorin (1989) on achieving coordination without communication.

${ }^{4}$ Another difficulty is that player 2 may avoid her "Stackelberg follower" action, for fear that playing it would cause 1 to reveal rationality, and in the ensuing full information subgame, they might play an equilibrium giving 2 an average discounted payoff that is less than her Stackelberg follower payoff. In our setting, the availability of binding contracts resolves this dilemma (see the closing paragraph of this section).
} 
tion effects by considering trembling-hand perfect equilibria (Aoyagi (1996)) or studying imperfect monitoring with a full-support assumption (Celentani, Fudenberg, Levine and Pesendorfer (1996)). We avoid these complications by assuming that whereas rational players may pretend to be behavioral, a behavioral type announces that type and does not pretend to be some other behavioral type. Thus rational player $i$ always knows that $j \neq i$ is either rational, or the particular behavioral type corresponding to the posture that $j$ originally declared.

When players are equally patient, reputation effects are much more likely to be overwhelmed by the multiplicity of possible expectations regarding continuation payoffs. Chan (2000) proves a folk theorem for repeated games with one informed and one uninformed player. In two exceptional cases, covered respectively by Chan (2000) and Cripps, Dekel and Pesendorfer (2005), reputational effects prevail. The case covered by Chan generalizes examples of Celentani et. al. (1996) and Cripps and Thomas (1997).

In our play-as-you-bargain model with enforceable contracts, some of the multiplicity of rational expectations one sees in repeated games is absent (although without reputational types, we show that a folk theorem for the efficiency frontier still applies). ${ }^{5}$ When $i$ offers $j$ a contract, $j$ knows exactly what will happen if she accepts it. We demonstrate that this is enough to produce essential determinacy of the division of surplus in the game. In an infinitely repeated game without contracts, $i$ has no way of guaranteeing $j$ a particular share of the future surplus. In a reputationally-perturbed version of that infinitely repeated game, if $i$ reveals rationality and $j$ does the same, they are in a subgame identical to the unperturbed game, and subject to the same vast multiplicity of equilibria. Abreu and Pearce (2002) give exogenous restrictions on continuation beliefs that suffice to pin down a particular division, and again it coincides with the "Nash bargaining with threats" allocations. We hope in future work to be able to dispense with those exogenous restrictions in the standard repeated game setting without contracts, by working with the renegotiation-proof equilibria proposed by Pearce (1989).

\footnotetext{
${ }^{5}$ The alternating offers protocol explored by Busch and Wen (1995) is less conducive to equilibrium multiplicity and a folk theorem does not apply. Nonetheless they give conditions under which multiple equilibria arise.
} 


\section{The Model}

In each round $n=0,1,2, \ldots$, the actions chosen in a finite game $G=$ $\left(S_{i}, U_{i}\right)_{i=1}^{2}$ determine the flow payoffs of players 1 and 2 . Thus, when players use actions $\left(s_{1}, s_{2}\right) \in\left(S_{1}, S_{2}\right)$, player $i$ 's payoff in that round is $U_{i}\left(s_{1}, s_{2}\right) \int_{0}^{1} e^{-r t} d t$ where $r>0$ is the common rate of interest. The overall payoff from an infinite stream is the present discounted value of the flow payoffs. If at any time players agree on a payoff pair in $\Pi$, the convex hull of the set of feasible payoffs of $G$, that flow payoff is realized for the remainder of the round and in all subsequent rounds: players sign an enforceable contract and there are no further strategic decisions. At the beginning of any round before agreement is reached, each player chooses a demand and action pair $\left(u_{i}, m_{i}\right) \in\left(\Pi_{i}, M_{i}\right)$, where $\Pi_{i}$ is the set of player $i$ 's feasible payoffs (the $i^{\text {th }}$ coordinate projection of $\Pi$ ) and $M_{i}$ is the set of mixed strategies in $G$. The players choose these pairs in some pre-specified order, which might be different in different periods (player 1 choosing first in odd periods, for example). Changing this exogenous ordering does not affect our results. We do not analyze the case in which the (demand, action) pairs are changed simultaneously.

While actions and demands can be changed only at integer times, one player's demands can be agreed to at any time $t \geq 0$ by the other player. ${ }^{6}$ A demand $u_{i}$ by player $i$ can be interpreted as an offer to $j \neq i$ of the best payoff for $j$ consistent with $i$ receiving $u_{i}$, which we denote by $\phi_{j}\left(u_{i}\right){ }^{7}$ Thus, an offer made at integer time $n$ is valid ("stands") until it is replaced by another offer (possibly the same) at $n+1$; a standing offer may be accepted at any time. Bargaining terminates at the first instant that offers made are mutually compatible or that a standing offer is accepted. An accepted offer is implemented instantaneously. If two standing offers are accepted at the same instant, the final agreement is taken to be either of the standing offers with equal probability. A similar tie-breaking rule applies when players make mutually compatible offers. Until agreement is reached, a player's choice of a (demand, action) pair at any $n>1$ can depend on the entire past history of (demand, action) pairs.

Each player is either "normal" (an optimizer) or with initial probability $z_{i}$, "behavioral". A behavioral player $i$ may be one of a finite set of types

\footnotetext{
${ }^{6}$ This mixture of discrete and continuous time simplifies the analysis of the "war of attrition" that arises, without causing problems with the definition of strategies and outcomes. We note that a more detailed variant of this hybrid model of time is introduced and used in Section 4.

${ }^{7}$ Since the stage game $G$ is finite, this best payoff is clearly well-defined.
} 
$\gamma_{i} \in \Gamma_{i}$. Each type is a strategy in the dynamic bargaining game. At the start of play a behavioral player $i$ announces (simultaneously with the other player) her true type $\gamma_{i} \in \Gamma_{i}$. We interpret this as an announcement of a bargaining posture. Let $\Pi^{*}$ be the set of strictly efficient and individually rational payoffs in the convex hull of feasible payoffs of the stage game, $G$ and $\Pi_{i}^{*}$ be the $i^{t h}$ coordinate projection of $\Pi^{*}$. Each $\gamma_{i} \in \Gamma_{i}$ is a machine defined by a finite set of states $Q_{i}$, an initial state $q_{i}^{0} \in Q_{i}$, an output function $\xi_{i}: Q_{i} \rightarrow\left(\Pi_{i}^{*} \times M_{i}\right)$, and a transition function $\psi_{i}: Q_{i} \times \Pi_{j}^{*} \times M_{j} \rightarrow Q_{i}{ }^{8}$ Denote by $\pi_{i}\left(\gamma_{i}\right)$ the (strictly positive) probability of posture/machine $\gamma_{i}$, conditional on player $i$ being behavioral. The set of postures and these conditional probabilities are held fixed throughout.

A normal player $i$ also announces a machine in $\Gamma_{i}$ as play begins, but of course she need not subsequently conform to her announcement. More generally, we could allow her to announce something outside $\Gamma_{i}$ or to keep quiet altogether. (This would not change our characterization results. See footnote 17 in section 4 . Nor would it affect the existence result in Section 5 , but it would necessitate some clumsy additions to the proof.) A normal player can condition $\left(u_{i}(n), m_{i}(n)\right)$, her choice of demand and mixed action in the $n^{\text {th }}$ round, on both players' initial announcements $\left(\gamma_{1}, \gamma_{2}\right)$ and on $\left(u_{l}(k), m_{l}(k)\right), l=1,2$ and $k=1, \ldots(n-1)$ (the history of play in the preceding rounds) and on $\left(u_{j}(n), m_{j}(n)\right)$ if $j$ moves before $i$ in period $n$. Notice this assumes a player's choice of mixed action is observable. One can interpret this to mean that a player has access to randomizing devices that can be verified ex post, and behavioral types use these devices when randomizing. ${ }^{9}$ A rational player imitating a behavioral type $\gamma_{i}$ will use these devices also, but in addition may (typically will, in equilibrium) conduct further, nonobservable randomization regarding whether or not to continue imitating $\gamma_{i}$. Players do not condition on the outcomes of the observable randomizing devices; allowing this would be akin to adding public randomization, which would have no impact on the result.

Interpreting the interval over which players can concede as the limit of a sequence of increasingly fine discrete divisions of time, we assume that if players adopt a pair of mixed actions $\left(m_{1}, m_{2}\right)$ in the $n^{\text {th }}$ round, as round $n$ progresses they experience the flow payoffs $\left(U_{1}\left(m_{1}, m_{2}\right)\right),\left(U_{2}\left(m_{1}, m_{2}\right)\right)$,

\footnotetext{
${ }^{8}$ The current state determines $i^{\prime} s$ behavior in round $n$, and hence there would be no gain in generality if the machine conditioned behavior in round $(n+1)$ upon its own past behavior.

${ }^{9}$ We invoke this assumption to simplify the analysis of situations in which a player's Nash threat involves randomization. There is no need for it in the large class of games in which both players' Nash threats are pure actions.
} 
rather than payoffs associated with the realization of a particular pure strategy pair. It is as if randomization were done not once at the beginning of the round, but over and over again.

For all $z=\left(z_{1}, z_{2}\right) \in(0,1)^{2}$, denote by $\mathcal{G}(z)$ the dynamic bargaining game described above, with initial probabilities $z_{i}, i=1,2$ that player $i$ is behavioral. Recall that conditional probabilities that $i$ is a certain type, given that she is behavioral, are held fixed.

\section{Stationary Postures}

This section studies the case where each behavioral type $\gamma_{i} \in M_{i}, i=$ 1,2 , is stationary, that is, $\gamma_{i}$ demands the same amount in any period, regardless of the history of play (and never accepts less), and plays the same action in every period until settlement is reached. These are the natural generalizations of the behavioral types of Myerson (1991) and Abreu and Gul (2000), to settings in which bargainers make payoff-relevant strategic choices in each period before reaching agreement. Whereas Abreu and Gul (2000) do a stationary perturbation of a bargaining game similar to that of Rubinstein (1982), with many behavioral types on each side, this section does the same sort of perturbation of the more complex bargaining problems of the kind introduced by Fernandez and Glazer (1991) and Haller and Holden (1990) and generalized by Busch and Wen (1995).

The equilibrium existence result of Section 5 applies immediately to this setting; we do not duplicate it here. At the heart of our characterization of equilibrium payoffs is the idea of "Nash bargaining with threats" (Nash (1953)), which is summarized below:

Recall the Nash (1950) bargaining solution for a convex non-empty bargaining set $\Pi \subseteq \mathbb{R}^{2}$, relative to a disagreement point $d \in \mathbb{R}^{2}$. The Nash bargaining solution, denoted $u^{N}(d)$, is the unique solution to the maximization problem

$$
\max _{u \in \Pi}\left(u_{1}-d_{1}\right)\left(u_{2}-d_{2}\right)
$$

when there exists $u \in \Pi$ s.t. $u \gg d$. If there does not, $u^{N}(d)$ is defined to be the strongly efficient point $u \in \Pi$ which satisfies $u \geq d$.

In Nash (1953) the above solution is derived as the unique limit of solutions to the non-cooperative Nash demand game when $\Pi$ is perturbed slightly and the perturbations go to zero. Nash's paper also endogenizes the choice of threats, and consequently disagreement point, and this second contribution plays a central role here. Starting with a game $G$, the bargaining set $\Pi$ 
is taken to be the convex hull of feasible payoffs of $G$. The threat point $d$ is determined as the non-cooperative (Nash) equilibrium of the following two "stage" game:

Stage 1 The two players independently choose (possibly mixed) threats $m_{i}, i=1,2$. The expected payoff from $\left(m_{1}, m_{2}\right)$ is the disagreement payoff, denoted $d\left(m_{1}, m_{2}\right)$.

Stage 2 The player's final payoffs are given by the Nash bargaining solution relative to the disagreement point determined in Stage 1.

Thus players choose threats to maximize their Stage 2 payoffs given the threats chosen by their opponent. Note that the set of player $i$ 's pure strategies in the threat game are her set of mixed strategies in the game $G$. Since the Nash bargaining solution yields a strongly efficient feasible payoff as a function of the threat point, the Nash threat game is strictly competitive in the space of pure strategies (of the threat game). Nash shows that the threat game has an equilibrium in pure strategies (i.e., players do not mix over mixed strategy threats), and consequently that all equilibria of the threat game are equivalent and interchangeable. In particular the threat game has a unique equilibrium payoff $\left(u_{1}^{*}, u_{2}^{*}\right)$ where $u^{*}=u^{N}\left(d\left(m_{1}^{*}, m_{2}^{*}\right)\right)$ and $m_{i}^{*}$ is an equilibrium threat for player $i$. To avoid distracting qualifications we assume henceforth that the stage game is non-degenerate in the sense that $u^{*}>d\left(m_{1}^{*}, m_{2}^{*}\right)$. Our solution essentially yields $\left(u_{1}^{*}, u_{2}^{*}\right)$ as the only equilibrium payoff which survives in the limit as the probability of behavioral types goes to zero.

We assume that one of the behavioral types on each side plays the "Nash bargaining with threats" (NBWT) strategy, demanding the Nash payoff and playing the Nash threat action. There are no restrictions on the demands and threats of all the other types that may be present; a clumsier assumption that would have essentially the same effect would be the requirement of a rich set of types on each side. The earliest analog of Assumption 1 in the reputational literature is the presence of a "Stackelberg leader" type in Fudenberg and Levine (1989).

Assumption 1 (NBWT) : For each player $i$, there exists $\gamma_{i}^{*} \in \Gamma_{i}$ such that in each period $\gamma_{i}^{*}$ demands $u_{i}^{*}$ (and accepts nothing less) and takes action $m_{i}^{*}$.

For a given stationary posture $\gamma_{i}$, let $u_{i}$ denote player $i$ 's stationary demand, and $m_{i}$ her stationary action. Recall that $\phi_{j}\left(u_{i}\right)$ is the corresponding 
offer to player $j$ (that is, $\left(u_{i}, \phi_{j}\left(u_{i}\right)\right)$ is an efficient feasible payoff in the stage game).

Assumption 2 : For all postures $\gamma_{i} \in \Gamma_{i}$

$$
\phi_{j}\left(u_{i}\right)>d_{j}\left(m_{j}^{\prime}, m_{i}\right) \quad \forall m_{j}^{\prime} \in M_{j} \quad i \neq j, \quad i, j=1,2 .
$$

Assumption 2 implies that postures penalize non-acceptance. That is, no matter what a player does when facing a particular behavioral type, she cannot get a flow payoff that is higher than what she has been offered. Lemma 1 will establish that being the first to reveal rationality is tantamount to conceding to one's opponent. ${ }^{10}$ This need not be true in the absence of Assumption 2; if, after a certain history which has revealed $i^{\prime} s$ rationanlity and left her fairly sure that $j$ is behavioral, $i$ might benefit from not conceding. No analogous assumption is required in the general non-stationary environment of Section 4, where we develop a quite different line of attack. But in Section 5, the proof of existence is facilitated by again assuming that postures penalize non-acceptance (see Assumption 4).

Lemma 1 Invoke Assumptions 1 and 2 and for any perfect Bayesian equilibrium $\sigma$, consider the continuation game following the choice of a pair of postures $\left(\gamma_{1}, \gamma_{2}\right)$, such that $u_{1}>\phi_{1}\left(u_{2}\right)$. Suppose that neither player has revealed rationality prior to time $t$ and that revealing rationality at $t$ (conditional upon neither player having revealed rationality earlier) is in the support of $j$ 's equilibrium strategy. Then if player $j$ reveals rationality at $t$ and $i$ does not, the resultant equilibrium continuation payoff is $\left(u_{i}, \phi_{j}\left(u_{i}\right)\right)$. Proof. See Appendix.

According to Lemma 2, once each side has adopted a posture, players concede with constant hazard rates. At no time other than 0 does anyone concede with strictly positive probability (as opposed to conditional density). For notational convenience, when particular postures and their associated mixed actions have been fixed, we write $\left(d_{1}, d_{2}\right)$ for the corresponding threat point.

\footnotetext{
${ }^{10}$ After revealing rationality and facing a possibly irrational opponent, a player is in a situation similar to that of the uninformed bargainer in Myerson (1991) (see the Introduction), or a durable goods monopolist facing a distribution of buyers with different valuations (see especially Coase (1972), Stokey (1981), Bulow (1982), Fudenberg, Levine and Tirole (1985), Gul, Sonnenschein and Wilson (1986) and the discussion in Abreu and Gul (2000), pp. 97-98 and 103-104).
} 
Let $\mu_{i}\left(\gamma_{i}\right)$ be the equilibrium probability with which player $i$ adopts the posture $\gamma_{i}$, conditional on $i$ being normal. Recall that $z_{i}$ is the prior probability that $i$ is behavioral, and $\pi_{i}\left(\gamma_{i}\right)$ is the probability that $i$ is of type $\gamma_{i}$, conditional on being behavioral. Let $\eta_{i}\left(\gamma_{i}\right)$ denote the posterior probability that a player $i$ who chooses $\gamma_{i}$ is behavioral. Then, by Bayes rule,

$$
\eta_{i}\left(\gamma_{i}\right)=\frac{z_{i} \pi_{i}\left(\gamma_{i}\right)}{z_{i} \pi_{i}\left(\gamma_{i}\right)+\left(1-z_{i}\right) \mu_{i}\left(\gamma_{i}\right)}
$$

When there is no danger of confusion, we will suppress the argument $\gamma_{i}$ in $\mu_{i}\left(\gamma_{i}\right), \pi_{i}\left(\gamma_{i}\right)$, and so on.

Lemma 2 Invoke Assumptions 1 and 2 and for any perfect Bayesian equilibrium $\sigma$, consider the continuation game following the choice of a pair of postures $\left(\gamma_{1}, \gamma_{2}\right)$, such that $u_{1}>\phi_{1}\left(u_{2}\right)$. This game has a unique perfect Bayesian equilibrium. In that equilibrium, at most one player concedes with positive probability at time zero. Thereafter, both players concede continuously with hazard rates $\lambda_{i}=\frac{r\left(\phi_{j}\left(u_{i}\right)-d_{j}\right)}{u_{j}-\phi_{j}\left(u_{i}\right)} i \neq j, i, j=1,2$ until some common time $T^{*}<\infty$ at which the posterior probability that each player $i$ is behavioral reaches 1 . Furthermore the probability with which player $j$ concedes to player $i$ at the beginning of the continuation game is $\max \left\{0,1-\frac{\eta_{j}}{\left(\eta_{i}\right)^{\lambda_{j} / \lambda_{i}}}\right\}$, where $\eta_{i}$ denotes the posterior probability that a player $i$ who chooses $\gamma_{i}$ is behavioral.

The proof is omitted. It is similar to Theorem 1 of Abreu and Gul (2000) and follows as a special case of the discussion in Section 4. We provide an intuitive treatment below.

Fixing an equilibrium $\sigma$ and postures $\left(\gamma_{1}, \gamma_{2}\right)$, denote by $F_{i}(t)$ the probability that player $i$ (unconditional upon whether $i$ is behavioral or normal) will reveal rationality by time $t$, conditional upon $j \neq i$ not revealing rationality prior to $t$. Since, by Lemma 1, the payoff to $i$ from revealing rationality is just what $j$ has offered her, the game (following the choice of postures) reduces to a war of attrition in which an opponent may be behavioral or rational.

Let $\lambda_{1}(t)=\frac{f_{1}(t)}{1-F_{1}(t)}$ denote 1's hazard rate of concession at $t>0$. This is calibrated to keep 2 indifferent between conceding at $t$ or $t+\Delta$. The cost to 2 of delaying concession is $\left(\phi_{2}\left(u_{1}\right)-d_{2}\right) \Delta$ while the benefit is $\frac{\left(u_{2}-\phi_{2}\left(u_{1}\right)\right)}{r} \lambda_{1}(t) \Delta$ (ignoring terms of order $\Delta^{2}$ and higher). Equating costs and benefits yields

$$
\lambda_{1}(t)=\frac{r\left(\phi_{2}\left(u_{1}\right)-d_{2}\right)}{u_{2}-\phi_{2}\left(u_{1}\right)} \equiv \lambda_{1}, \quad \text { a constant independent of } t .
$$


Hence,

$$
1-F_{2}(t)=c_{2} e^{-\lambda_{2} t}
$$

where $c_{2} \in(0,1]$ is a constant of integration to be determined by equilibrium conditions. Observe that $F_{j}(0)=1-c_{j}$, where $F_{j}(0)$ is the probability with which $j$ concedes at $t=0$. Clearly $c_{i} \in[0,1]$ and $\left(1-c_{1}\right)\left(1-c_{2}\right)>0$.

Since behavioral types never concede, we require that

$$
1-F_{i}(t) \geq \eta_{i} \text { all } t \geq 0,
$$

where $\eta_{i}$ is the posterior probability that player $i$ who chooses posture $\gamma_{i}$ is behavioral.

The above requirements pin down the equilibrium uniquely. It follows from the latter condition that a normal player $i$ must concede with probability 1 in finite time, indeed, at the latest, by $T_{i}$ where

$$
\begin{aligned}
e^{-\lambda_{i} T_{i}} & =\eta_{i} \text { and } \\
T_{i} & =\frac{-\log \eta_{i}}{\lambda_{i}}
\end{aligned}
$$

is the instant by which normal $i$ would finish conceding if $c_{i}=1$, or equivalently if player $i$ did not concede with positive probability at $t=0$. In equilibrium, normal types of both players must finish conceding at the same instant, and at most one player can concede with positive probability at $t=0$.

Let $T^{*}=\min \left\{T_{1}, T_{2}\right\}$. If $T_{i}=T^{*}$ then $c_{i}=1$ and $c_{j} \in(0,1]$ is determined by the requirement that

$$
\begin{aligned}
1-F_{j}(t) & =c_{j} e^{-\lambda_{i} T^{*}}=\eta_{j} \\
& \Longrightarrow 1-c_{j}=F_{j}(0)=1-\frac{\eta_{j}}{\left(\eta_{i}\right)^{\lambda_{j} / \lambda_{i}}}
\end{aligned}
$$

More generally,

$$
F_{j}(0)=\max \left\{0,1-\frac{\eta_{j}}{\left(\eta_{i}\right)^{\lambda_{j} / \lambda_{i}}}\right\}
$$

independently of whether $T_{j}<T_{i}$ or $T_{j} \geq T_{i}$.

Let $\eta_{i}(t)$ denote the posterior probability that player $i$ is behavioral, absent concession until time $t$. Then $\eta_{i}(t)=\frac{\eta_{i}}{1-F_{i}(t)}=\frac{1}{c_{i}} \eta_{i} e^{\lambda_{i} t}$. That is, $\lambda_{i}$ is the rate of growth of player $i$ 's reputation (for being behavioral). If $T_{i}>T^{*}$, then $c_{i}$ is less than 1 , and is chosen to boost $i$ 's reputation 
(conditional upon non-concession at $t=0$ ) by just enough for both players' reputations to reach 1 simultaneously at $T^{*}$.

It follows that the player with the larger concession hazard rate, ceteris paribus, is at an advantage in the war of attrition. Suppose for example that in equilibrium, after adopting some particular pair of profiles, players have the same initial reputations, and $\lambda_{1}>\lambda_{2}$. Suppose further (counterfactually, as we shall see) that neither player concedes with positive probability at time 0. Player 1's reputation will reach 1 before 2's reputation does, in violation of Lemma 2. The only way to keep this from happening is for 2 to concede with enough probability at time zero so that in the event that she is observed not to have conceded, her reputation jumps just enough that the two players' reputations will reach 1 together after all. If initial reputations are tiny, even a small difference in hazard rates must be compensated for by concession at zero with probability close to 1 . This follows from the formula for $F_{j}(0)$ given above.

Naive intuition might suggest that player $i$ will tend to imitate the greediest possible type. But the formula in Lemma 2 indicates that by moderating the demand, $i$ increases $\lambda_{i}$ and decreases $\lambda_{j}$, which may serve $i$ better in the war of attrition. The formula further shows that $i$ should choose an action (while waiting) that hurts the opponent $j$ without hurting $i$ too much. Of course that is also what a player has in mind when choosing a threat in the Nash bargaining with threats (NBWT) game. The connection can be made precise as follows.

Lemma 3 Suppose that player 1 adopts his NBWT posture. Then for all postures 2 could adopt, except ones that give 1 at least as much as he is asking for, $\lambda_{1}>\lambda_{2}$.

Proof. This is most easily seen graphically. Let 1 adopt the NBWT position $\gamma_{i}^{*}=\left(u_{i}^{*}, m_{i}^{*}\right)$ and 2 adopt any posture $\gamma_{2}=\left(u_{2}, m_{2}\right)$ with $u_{2}>u_{2}^{*}$. The NBWT threat point and allocation are denoted $d^{*}$ and $u^{*}$, respectively. Let $d \equiv d\left(m_{1}^{*}, m_{2}\right)$ and $u \equiv\left(\phi_{1}\left(u_{2}\right), u_{2}\right)$. See Figure 1 .

By Assumption 2, $d_{1}<\phi_{1}\left(u_{2}\right)$. Since $\left(m_{1}^{*}, m_{2}^{*}\right)$ is an equilibrium of the Nash threat game, $d$ lies on or below the line through $d^{*} u^{*}$ (if not, $m_{2}$ would be a strictly improving deviation for player 2 in the Nash threat game). By Nash's (1950) characterization of the Nash bargaining solution, the slope of the line $d^{*} u^{*}$ equals the absolute value of the slope of some supporting hyperplane to the set $\Pi$ (the convex hull of the feasible set of $G$ ) at $u^{*}$. Hence slope $d e>$ slope $d u^{*} \geq$ slope $d^{*} u^{*} \geq \mid$ slope $u u^{*} \mid$. 
But

$$
\lambda_{1}=\frac{r\left(u_{2}^{*}-d_{2}\right)}{u_{2}-u_{2}^{*}}>\frac{r\left(u_{1}-d_{1}\right)}{u_{1}^{*}-\phi_{1}\left(u_{2}\right)}=\lambda_{2}
$$

if and only if

$$
\text { slope } d e>\mid \text { slope } u u^{*} \mid \text {. }
$$

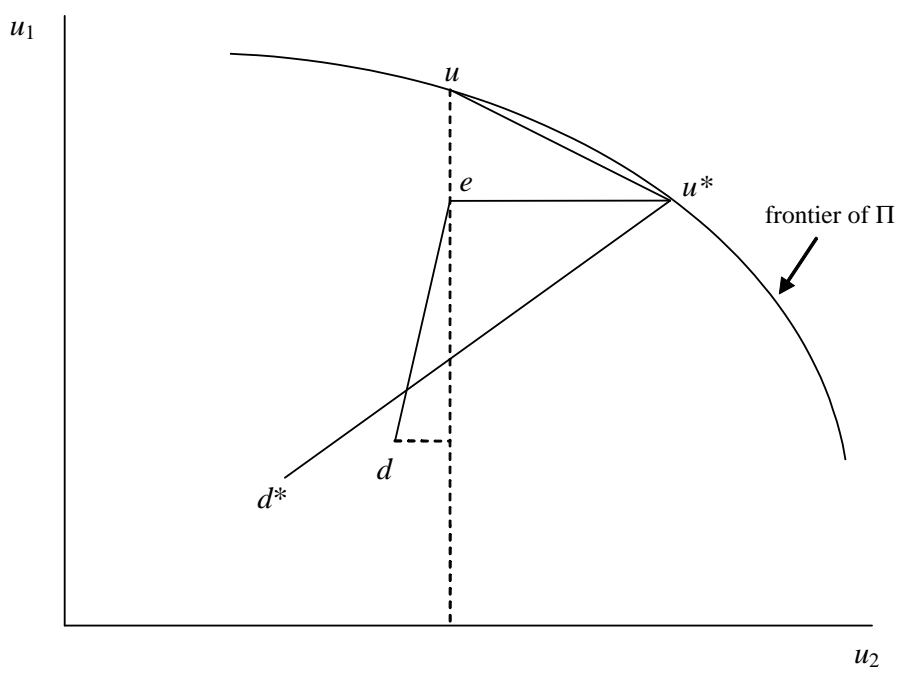

Figure 1

If normal player 2 adopts a particular posture with extremely low probability in equilibrium, her reputation jumps dramatically when she is observed to adopt the posture in question. This gives her a major advantage in the ensuing war of attrition. But given any lower bound on this probability, and any upper bound on the ratio of any two ex ante probabilities (of behavioral types), the latter probabilities $z_{1}, z_{2}$ can be chosen small enough so that in the continuation game following that choice of posture, 1's expected payoff is close to (or greater than) his Nash bargaining with threats payoff.

Lemma 4 Invoke Assumptions 1 and 2. For any $\xi>0, R \in(0, \infty)$ and $\bar{\mu}>0$ there exists $\delta>0$ such that if $z_{i} \leq \delta, i=1,2$ and $\max \left\{\frac{z_{1}}{z_{2}}, \frac{z_{2}}{z_{1}}\right\} \leq R$, then for any perfect Bayesian equilibrium $\sigma$ the payoff to a rational player 1 in the continuation game $\left(\gamma_{1}^{*}, \gamma_{2}\right)$ is at least $\left(u_{1}^{*}-\xi / 2\right)$ for any $\gamma_{2} \in \Gamma_{2}$ which a rational player 2 adopts in equilibrium with probability $\mu_{2}\left(\gamma_{2}\right) \geq \bar{\mu}$. Proof. Consider the continuation game with $\left(\gamma_{1}^{*}, \gamma_{2}\right)$. By Lemma 3, either $\gamma_{2}$ entails $u_{2}$ with $\phi_{1}\left(u_{2}\right) \geq u_{1}^{*}$, or $\lambda_{1}>\lambda_{2}$. Suppose $u_{1}^{*}>\phi_{1}\left(u_{2}\right)$ and that 
rational 2 adopts $\gamma_{2}$ with at least probability $\underline{\mu}>0$. Then

$$
\begin{aligned}
\eta_{1} & \geq \frac{z_{1} \pi_{1}\left(\gamma_{1}\right)}{\left(1-z_{1}\right) \cdot 1+z_{1} \pi_{1}\left(\gamma_{1}\right)} \\
\eta_{2} & \leq \frac{z_{2} \pi_{2}\left(\gamma_{2}\right)}{\left(1-z_{2}\right) \cdot \bar{\mu}+z_{2} \pi_{2}\left(\gamma_{2}\right)} \leq z_{2} B \\
& \Rightarrow \frac{\eta_{2}}{\eta_{1}} \leq \frac{z_{2}}{z_{1}} \cdot \frac{\pi_{2}\left(\gamma_{2}\right)}{\pi_{1}\left(\gamma_{1}\right)} \cdot \frac{\left(1-z_{1}\right)+z_{1} \pi_{1}\left(\gamma_{1}\right)}{\left(1-z_{2}\right) \cdot \bar{\mu}+z_{2} \pi_{2}\left(\gamma_{2}\right)} \leq R C
\end{aligned}
$$

for given $R$ and some finite constants $B, C$ independent of $\left(z_{1}, z_{2}\right)$. Recall that the conditional probabilities $\pi_{i}\left(\gamma_{i}\right)$ are exogenous constants.

From Lemma 2,

$$
F_{2}(0)=1-\frac{\eta_{2}}{\eta_{1}}\left(\eta_{1}\right)^{1-\frac{\lambda_{2}}{\lambda_{1}}}
$$

if the latter term is non-negative. By the preceding inequalities,

$$
\begin{aligned}
F_{2}(0) & \geq 1-R C\left(z_{2} B\right)^{1-\frac{\lambda_{2}}{\lambda_{1}}} \\
& \geq 1-\bar{R} \delta^{1-\frac{\lambda_{2}}{\lambda_{1}}}
\end{aligned}
$$

where $\bar{R}=R C B^{1-\frac{\lambda_{2}}{\lambda_{1}}}<\infty$. Hence for $\delta$ small enough, $F_{2}(0)$ is close to 1 .

Player 1's payoff is:

$$
\begin{aligned}
& F_{2}(0) u_{1}^{*}+\left(1-F_{2}(0)\right) \phi_{1}\left(u_{2}\right) \\
\geq & u_{1}^{*}-\frac{\xi}{2}
\end{aligned}
$$

for $\delta$ small enough and (consequently) $F_{2}(0)$ close enough to 1.

Suppose that player 1 adopts his NBWT posture. When he meets a type that 2 chooses extremely rarely, Lemma 4 does not apply; but since this happens so rarely, it has negligible influence on the weighted average that determines 1's expected payoff. In all other cases, Lemma 4 guarantees him virtually his NBWT payoff. Theorem 1 and its proof make this precise.

Theorem 1 Invoke Assumptions 1 and 2. Then for any $\varepsilon>0$ and $R \in$ $(0, \infty)$ there exists $\delta>0$ such that if $z_{i} \leq \delta, i=1,2$ and $\max \left\{\frac{z_{1}}{z_{2}}, \frac{z_{2}}{z_{1}}\right\} \leq R$ then for any perfect Bayesian equilibrium $\sigma$ of $\mathcal{G}(z),\left|U(\sigma)-u^{*}\right|<\varepsilon$.

Proof. For any given perfect Bayesian equilibrium $\sigma$, and $\bar{\mu}>0$, let $\widehat{\Gamma}_{2}=\left\{\gamma_{2} \in \Gamma_{2} \mid \mu_{2}\left(\gamma_{2}\right) \leq \bar{\mu}\right\}$. Then $\sum_{\gamma_{2} \in \widehat{\Gamma}_{2}} \mu_{2}\left(\gamma_{2}\right) \leq\left|\widehat{\Gamma}_{2}\right| \bar{\mu} \leq\left|\Gamma_{2}\right| \bar{\mu}$. Hence 
$\sum_{\gamma_{2} \in \Gamma_{2} / \widehat{\Gamma}_{2}} \mu_{2}\left(\gamma_{2}\right)=1-\sum_{\gamma_{2} \in \widehat{\Gamma}_{2}} \mu_{2}\left(\gamma_{2}\right) \geq 1-\left|\Gamma_{2}\right| \bar{\mu}$. Under the conditions of Lemma 4 , for any $\gamma_{2} \in \Gamma_{2} / \widehat{\Gamma}_{2}$, the payoff to a rational player 1 in the continuation game $\left(\gamma_{1}^{*}, \gamma_{2}\right)$ is at least $\left(u_{1}^{*}-\xi / 2\right)$, and consequently the payoff to adopting $\gamma_{1}^{*}$ is at least

$$
\left(1-\left|\Gamma_{2}\right| \bar{\mu}\right)\left(u_{2}^{*}-\frac{\xi}{2}\right)+\left|\Gamma_{2}\right| \bar{\mu} \underline{w}_{i}
$$

where $\underline{w}_{i}$ is the lowest payoff to $i$ in the (finite) stage game $\mathrm{G}$.

Clearly we can choose $\bar{\mu}>0$ such that $\left|\Gamma_{2}\right| \bar{\mu} \leq 1$ and

$$
\left(1-\left|\Gamma_{2}\right| \bar{\mu}\right)\left(u_{2}^{*}-\frac{\xi}{2}\right)+\left|\Gamma_{2}\right| \bar{\mu} \underline{w}_{i} \geq u_{2}^{*}-\xi
$$

For such a $\bar{\mu}>0$ Lemma 4 immediately implies that under the stated conditions, the payoff to adopting $\gamma_{1}^{*}$ is at least $u_{1}^{*}-\xi$, in any $\operatorname{PBE} \sigma$. It follows that $U_{1}(\sigma) \geq u_{1}^{*}-\xi$. This is true for both players and $u^{*}$ is a (strongly) efficient feasible payoff of the stage game. ${ }^{11}$ Hence the Theorem follows directly.

In summary, when a repeated game with contracts is perturbed slightly by the addition of stationary behavioral types on each side, the continuum of perfect Bayesian equilibria in the unperturbed game is replaced by a precise prediction about how surplus is shared. The prediction is virtually independent of the ex-ante distribution over behavioral types, as long as the NBWT type is included on each side. With probability close to 1 , the demands made by each side, and the actions taken while waiting, are those suggested by Nash (1953) in a much simpler context. Interestingly, Theorem 1 does not require $r$, the rate of interest, to be close to zero. If $r$ is relatively high, concession hazard rates $\lambda_{1}$ and $\lambda_{2}$ will be correspondingly high, to make the players indifferent between waiting or conceding.

\section{Nonstationary Postures}

Following Fernandez and Glazer (1991) and Haller and Holden (1990), Busch and Wen (1995) have provided a general analysis for repeated games with complete information where a long-run enforceable contract can be signed.

\footnotetext{
${ }^{11}$ That is, there does not exist feasible $u^{\prime}$ s.t. $u_{i}^{\prime}>u_{i}^{*}$ and $u_{j}^{\prime} \geq u_{j}^{*}$.
} 
In conformity with their results, in many games there is a significant multiplicity of equilibrium outcomes. ${ }^{12}$ Our goal is to be able to say that any rich perturbation of such a game leads to an essentially unique outcome, and that the outcome is not sensitive to the small ex-ante probabilities of the respective behavioral types. That is true if perturbations are restricted to stationary strategies, as Section 3 has shown. Which of the results there survive the introduction of nonstationary strategies?

We revert now to the general model specified in Section 2. Behavioral types are finite automata that announce and follow repeated game strategies that may have complicated intertemporal features and can respond to the opponent's play. Suppose one asks how well player 1's stationary Nash bargaining with threats (NBWT) strategy would do against any nonstationary posture 2 might adopt. How different from Section 3 would the analysis look, and does 1 do himself harm by not taking advantage of the opportunity to use a dynamic closed-loop strategy himself?

We formulate a new hybrid discrete/continuous model of time that simplifies the war of attrition calculations without introducing any of the logical difficulties associated with games played in continuous time. It would appear that a natural way to accomplish this is to restrict players to changing their offers and actions at discrete intervals (say, at integer times), while allowing them to accept the opponent's offer at any moment (in continuous time). It is necessary to elaborate this model slightly, to avoid "openness" problems. In the event that player $j$ responds to player $i$ 's offer at time 5 with an offer that $i$ considers attractive, $i$ may want to accept $j$ 's offer "as soon as possible", at the first moment following 5 , as it were. Similarly, if $i$ 's offer to $j$ decreases at 5 , it is natural to provide a last time for $j$ to accept the more generous offer. To accommodate this formally, we split the integer time 5 into four "dates" which we call $(5,-2),(5,-1),(5,1)$ and $(5,2)$. The date $(5,-2)$ is the last time at which players can accept offers made at time 4. If $i$ is the player specified to make her offer first at time 5 , she does so at date $(5,-1)$. Player $j \neq i$ then makes his offer at $(5,1)$, and players get their first opportunities to accept the new offers at $(5,2)$. While these four dates are sequential from a logical point of view, they are considered to occur at calendar time 5, so no discounting occurs between them. ${ }^{13}$ This

\footnotetext{
${ }^{12}$ In our formulation there is no discounting between the offers of 1 and 2 , and offers which are on the table can be accepted simultaneously by both players. This makes it easy to establish a folk theorem result (see Section 5).

${ }^{13}$ To interpret this model, think of a setting in which players accept offers in continuous time, except in the "time-out" pauses (open intervals) during which new actions and offers are chosen. Then let the duration of each time-out approach zero.
} 
device simply ensures that the set of times at which a player can accept an offer on the table, is compact.

We now introduce the notation regarding time that is used in the argument to follow. Our primitive notion of time is a "date". The set of dates is $\mathcal{T}$. A date $\tau \in \mathcal{T}$ has two dimensions; $\tau=(t, k)$. For $\tau \in \mathcal{T}$ let $t(\tau)$ denote the first dimension and $k(\tau)$ the second. The first dimension $t(\tau)$ specifies the calendar time at which date $\tau$ occurs. The second dimension allows us to order different events that occur at the same calendar time, as explained in the preceding paragraph. Only for integer time is the "splitting" discussed above needed. Hence, for $n \in \mathcal{N} \equiv\{0,1,2, \ldots\}$, $\{(n,-2),(n,-1),(n,+1),(n,+2)\} \subseteq \mathcal{T}$. For $t \notin \mathcal{N},(t, k) \in \mathcal{T}$ if and only if $t>0$ and $k=0$. At dates $(n,-1)$ and $(n,+1), n \in \mathcal{N}$, players can make new (offer, action) choices in an arbitrary pre-specified order. The new offer can be accepted at dates $(n,+1),((n+1),-1)$ and all dates in between. Thus the end of a round and the beginning of the next round are distinct. Discounting depends only on the pure time component of a date. The ordering on $\mathcal{T}$ is lexicographic: for any $\tau, \tau^{\prime} \in \mathcal{T}, \tau^{\prime} \equiv\left(t^{\prime}, k^{\prime}\right) \succ(t, k) \equiv \tau$ if $t^{\prime}>t$ or if $t^{\prime}=t$ and $k^{\prime}>k$. A player's choices at date $(n,+1)$, say, can be conditioned on observed choices at dates $(n,-1),(n,-2)$ and, of course, all preceding dates. For $i=1,2$ and $n \in \mathcal{N}$ we define $k_{i}^{n}$ to equal -1 or +1 depending upon whether $i$ has the move at $(n,-1)$ or $(n,+1)$.

For later reference, we define the infimum of a set of dates $\Omega \subseteq \mathcal{T}$. Denote $\underline{\omega} \equiv \inf \Omega$. Let $\Omega_{t}=\{t(\omega) \mid \omega \in \Omega\}$ and $\underline{t} \equiv \inf \Omega_{t}$. If $\underline{t} \notin \mathcal{N}$, then $\underline{\omega} \equiv(\underline{t}, 0)$. If $\underline{t} \in \mathcal{N}$, define $\Omega(\underline{t})=\{\omega \in \Omega \mid t(\omega)=\underline{t}\}$. If $\Omega(\underline{t}) \neq \emptyset$ then $\underline{\omega} \equiv\left\{\omega \in \Omega(\underline{t}) \mid k(\omega) \leq k\left(\omega^{\prime}\right)\right.$ all $\left.\omega^{\prime} \in \Omega(\underline{t})\right\}$. If $\Omega(\underline{t})=\emptyset$ then $\underline{\omega} \equiv(\underline{t},+2)$. The supremum is defined analogously. These are the natural extensions of the usual definitions.

With 1's strategy fixed at the stationary NBWT action and demand, player 2's situation is similar in some ways to what she faced in Section 3. Whenever 2 reveals rationality, one can show that she does so by, in effect, accepting 1's offer. This is the one-sided analog of Lemma 1 in Section 3. But the same is not true for player 1, who faces a nonstationary type. Suppose that 1 is offered 5 until some date $\tau$, and 10 thereafter. Rather than wait to get 10 at $\tau$, at an earlier time $\tau^{\prime}$ he might offer a Pareto-superior contract: give me 9 right now. Player 2 might accept this (as long as she doesn't expect to do better in the subgame in which she instead reveals rationality without accepting 1's offer). Thus, the offer from 2's machine $\gamma_{2}$ at $\tau^{\prime}$ is just a lower bound on 1's equilibrium expectation of the payoffs he would receive if $\tau^{\prime}$ arrives without either player having revealed rationality.

The reader may wonder why 1 would wait until $\tau^{\prime}$ to make this sugges- 
tion, and for that matter, why 1 doesn't ask for an even greater amount. The answer lies in the full-information subgames after 1 and 2 have both revealed rationality. These typically have a continuum of subgame perfect equilibria, and in the construction of a solution of the full game, the selection from this set can depend on arbitrary details of the history of play. For example, if 1 demands 9 at $\tau^{\prime \prime}$ prior to $\tau^{\prime}$ instead of at $\tau^{\prime}$, or 9.3 at $\tau$, say, 2 could believe that she would fare extremely well, and 1 badly, if she revealed rationality instead of accepting 1's offer.

The above example might leave the impression that 1's expected payoff at $\tau^{\prime}$ could exceed 2's offer there only because $\gamma_{2}$ later makes a more generous offer in response to 1's constant play of his NBWT position. This is not true. For example, because 2's behavioral type may make offers that depend on 1 's past offer or actions, 1 may be able to induce more generous offers from 2 by departing from his NBWT posture. At $\tau$, for example, if 1 reveals rationality without accepting the offer of 10 , he may be able to manipulate $\gamma_{2}$ into offering him 15. His expected payoff at $\tau$ could therefore easily exceed 10.

To summarize, when 1's static NBWT strategy faces more complex strategies of 2, 1's expected payoff in a particular continuation game is no longer given by what 2 offers him, and may vary greatly across different equilibria of that continuation game. A normal player 1 may want to reveal rationality (by abandoning the NBWT posture at some point) but not accept 2's offer. Further we shall see that nonstationarity in 2's posture induces discontinuities in the war of attrition, with one or more players conceding away from time zero with strictly positive probability.

All of the above makes it impossible to replicate the line of attack of Section 3. Perhaps surprisingly, the main result concerning players' payoffs is essentially unchanged, along with the power of the static NBWT posture. The proofs, however, are quite different, and much more elaborate. This Section states and proves our main result, Theorem 2 .

Let $u_{i}\left(\tau \mid \gamma_{1}, \gamma_{2}\right)$ denote player $i$ 's demand at time $\tau$, and $d_{i}\left(\tau \mid \gamma_{1}, \gamma_{2}\right)$ denote the flow payoff to $i$ at $\tau$, given that both players conform with $\left(\gamma_{1}, \gamma_{2}\right)$ until $\tau$. So far for integer $n$ we have not introduced a date $(n, 0)$. It will be convenient later to define $d_{i}\left((n, 0) \mid \gamma_{1}, \gamma_{2}\right)$. We set $d_{i}\left((n, 0) \mid \gamma_{1}, \gamma_{2}\right) \equiv$ $d_{i}\left((n,+2) \mid \gamma_{1}, \gamma_{2}\right)$. When there is no danger of confusion, we will drop the arguments $\gamma_{1}, \gamma_{2}$.

For the profile of postures $\left(\gamma_{1}^{*}, \gamma_{2}\right)$, take as given all elements of $\gamma_{2}$ except the mapping from the finite set of states to demands. If the latter demands are chosen randomly, ties between demands and flow payoffs will occur with probability zero. When demands exactly equal flow payoffs, potential inde- 
terminacies in 1's response create a profusion of cases to be treated. We avoid this by making the following genericity assumption.

Assumption 3 (Generic Types) For all $\gamma_{2} \in \Gamma_{2}$ consider the continuation game defined by $\left(\gamma_{1}^{*}, \gamma_{2}\right)$. For all $n, \phi_{1}\left(u_{2}\left((n,+2) \mid \gamma_{1}^{*}, \gamma_{2}\right)\right) \neq d_{1}\left((n,+2) \mid \gamma_{1}^{*}, \gamma_{2}\right)$. $A$ corresponding assumption applies to types $\gamma_{1} \in \Gamma_{1}$.

Theorem 2 Invoke Assumptions 1 and 3. Then for any $\varepsilon>0$ and $R \in$ $(0, \infty)$ there exists $\delta>0$ such that if $z_{i} \leq \delta, i=1,2$ and $\max \left\{\frac{z_{1}}{z_{2}}, \frac{z_{2}}{z_{1}}\right\} \leq R$, then for any perfect Bayesian equilibrium $\sigma$ of $\mathcal{G}(z),\left|U(\sigma)-u^{*}\right|<\varepsilon$.

Theorem 2 says that no matter how high you allow the bound on the relative probabilities that the respective players are behavioral to be, and no matter how close to $u_{1}^{*}$ you want 1's expected utility to be, this is achieved uniformly across all perfect Bayesian equilibria, when behavioral players have sufficiently low prior probabilities.

Before providing the proof, we give a quick account of the main ideas. Given the unavoidable fact that a typical continuation game (following the choice of postures) suffers from a vast multiplicity of perfect Bayesian equilibria, our strategy is as follows. Any particular equilibrium of the full game offers player 1 expected payoffs at each date in each continuation game, following the realization of 2 's choice of posture $\gamma_{2}$. Just as one can graph the offers $\gamma_{2}$ makes to 1 over time, one can graph the payoffs 1 would get by first revealing rationality at any date $\left(n, k_{1}^{n}\right)$, by departing from the (offer, action) pair as given by the initial posture $\gamma_{1}$ (in the case under consideration $\gamma_{1}^{*}$, of course) in interaction with the opponent's posture $\gamma_{2}$. It is the maximum of these two values that drives the war of attrition. In analyzing that war of attrition, one can treat the stream of these maxima as exogenous variation, just as one accepts the possibility of arbitrary strategies $\gamma_{2}$. Once the characterization result is established for all possible streams, it holds a fortiori for all graphs that could actually arise in equilibrium. ${ }^{14}$

Recall from Section 3 that the more player $i$ demands, the slower $i$ 's rate of concession must be, and the slower $i$ 's reputation will grow. If $i$ 's

\footnotetext{
${ }^{14}$ More precisely, Lemma 11 will establish a uniform upper bound on the maxima in question, for the interval of dates relevant for our arguments. Our characterization result holds for any stream satisfying the upper bound, so it is not necessary to figure out exactly which streams could actually arise in equilibrium.
} 
demand is sufficiently greedy, this will require $i$ to concede at time 0 with high probability. The same basic force is at work here. If 2 is asking for more than her NBWT payoff, she has to concede slower than 1 (if he chooses his NBWT posture). The rate changes as her demands change, and one has to integrate these rates and add them to discrete probability concessions. ${ }^{15}$ It is necessary to make cross-player comparisons of payoff discontinuities of different sizes and with qualitatively different effects. This is the most delicate part of the argument. But the same picture ultimately emerges: over all, 2's reputation grows more slowly than 1's and this becomes decisive when prior behavioral probabilities are low.

Non-stationarities in player 2's posture typically induce discrete concession episodes by both players. The simplest case, which we call a "downward jump", involves a decrease in the value of 2's offer to 1 . Suppose that at date $\left(n, k_{2}^{n}\right)$ before $\tau^{*}$, player 2's offer falls from $a$ to $b<a$. If 1 ever accepts the offer of $b$ in equilibrium immediately after $(n,+2)$, he must be compensated at $(n,+2)$ for letting the offer fall from $a$ to $b$, by a probabilistic concession from 2 . The probability $P_{2}$ of 2 's conceding at $(n,+2)$ that makes 1 indifferent between accepting the offer of $a$ or waiting satisfies: ${ }^{16}$

$$
a=P_{2} u_{1}^{*}+\left(1-P_{2}\right) b
$$

"Upward jumps" have more interesting repercussions. Assume for simplicity that 2's action choice is constant and that at some date $\tau \in\left(0, \tau^{*}\right]$, 2 's offer jumps up from $b$ to $a>b$ (or alternatively, that at $\tau$, the equilibrium implicitly offers 1 the payoff $a$ for revealing rationality at $\tau$ without accepting 2's offer). For some time interval of length $\Delta$ before $\tau, 1$ would rather wait until $\tau$ to get $a$, than to concede immediately and get $b$ (see Figure 2). Since 1 experiences flow payoffs while waiting, $\Delta$ solves:

$$
\frac{b}{r}=\int_{t_{1}-\Delta}^{t_{1}} d_{1}((s, 0)) e^{-r\left(s-\left(t_{1}-\Delta\right)\right)} d s+e^{-r \Delta} \frac{a}{r}
$$

where $t_{1} \equiv t(\tau)$ and $d_{1}((s, 0))$ is player 1's flow payoff (given $\left(\gamma_{1}^{*}, \gamma_{2}\right)$ ) at

\footnotetext{
${ }^{15}$ The paragraphs below explain how increases in 2 's offer at $\left(n, k_{2}^{n}\right)$ induce discrete concessions by 1 at time $n$ and decreases in 2's offer induce discrete concessions by 2 .

${ }^{16}$ As noted earlier, player 1's expected payoff at some date may exceed what 2 offers him there. A fall in this expected value will induce a compensating discrete concession by 2 , even in the absence of any change in 2's offer. The initial higher value could be 1's payoff to revealing rationality at $\left(n, k_{1}^{n}\right)$. And the lower value might itself exceed 2's offer, because it might be 1's present discounted value from waiting for a superior offer 2 will make later (see the discussion of "shadows" three paragraphs below).
} 
date $(s, 0)$.

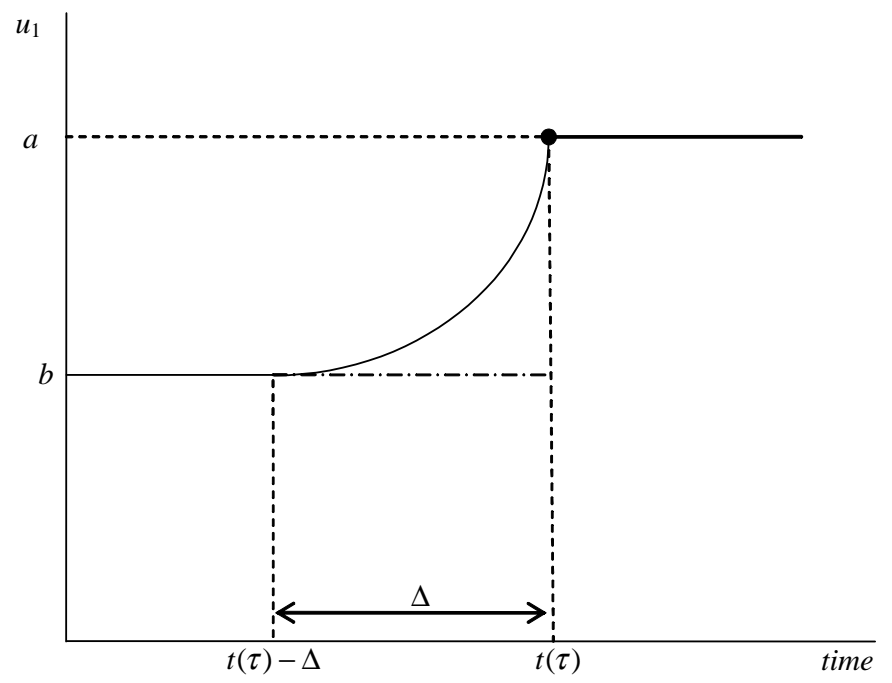

Figure 2

Notice that 2 will not concede in the $\Delta$ interval before $t(\tau)$ either: since 1 never concedes in that interval, 2 is strictly better off conceding at the start of the interval than at any point in its interior. For 2 to be just compensated for waiting through the barren interval $\Delta, 1$ must concede at $t(\tau)$ with (conditional) probability $P_{1}$ solving:

$$
\frac{u_{2}^{*}}{r}=\int_{t_{0}}^{t_{1}} d_{2}((s, 0)) e^{-r\left(s-t_{0}\right)} d s+e^{-r\left(t_{1}-t_{0}\right)}\left[P_{1} \frac{v_{2}^{2}(\tau)}{r}+\left(1-P_{1}\right) \frac{u_{2}^{*}}{r}\right]
$$

where $t_{0} \equiv t_{1}-\Delta$ and $v_{2}^{2}(\tau)$ is normal player 2's payoff when player 1 reveals rationality at $\tau$.

We say that the jump at $\tau$ "casts a shadow" of length $\Delta$ over the time period preceding $\tau$. What if no $P_{1} \leq 1$ solves the equation? Then 2 cannot be induced to wait, and normal 2 should concede with probability 1 weakly before the shadow begins (contradicting our assumption that $\tau \in\left(0, \tau^{*}\right]$ ). This expression makes it clear that changes in flow payoffs $d_{1}((s, 0))$ can also contribute to or even cause "shadows". For instance even if $b=a$, if there are changes in 2's action choices so that initially player 1's flow payoffs $d_{1}((s, 0))$ are less than $a$ and later $d_{1}((s, 0))$ exceeds $a$, so that

$$
\int_{t_{1}-\Delta}^{t_{1}} d_{1}((s, 0)) e^{-r\left(s-\left(t_{1}-\Delta\right)\right.} d s=a \int_{t_{1}-\Delta}^{t_{1}} e^{-r\left(s-\left(t_{1}-\Delta\right)\right.} d s
$$


then we have a shadow of length $\Delta$ generated exclusively by changes in flow payoffs.

Interestingly, there can be an upward jump at $\tau$, followed by a downward jump "at the same instant". Suppose that 2's posture $\gamma_{2}$ is as illustrated in Figure 2 above, but that the equilibrium offers $c>a$ at $\tau \equiv(n, k)$, $k \in\{-1,+1\}$ (and nowhere else, for simplicity). Clearly 1's option of getting $c$ at $(n, k)$ casts a shadow (a longer one than that cast by $a$ ) over an interval in which neither 1 nor 2 will concede. Player 1 reveals rationality probabilistically at $(n, k)$ (without accepting 2 's offer), to compensate 2 for waiting through the barren period. In the event that he does not concede, he faces an immediate drop in expected payoff from $c$ to $a$. To make 1 indifferent between revealing rationality and waiting, 2 must concede with probability

$$
P_{2}=\frac{c-a}{u_{1}^{*}-a}
$$

conditional on 1's not revealing rationality at $(n, k)$.

\section{Proof of Theorem 2.}

Theorem 2, the analog of Theorem 1, follows from Lemma 5 below in the same way as Theorem 1 follows from Lemma 4 (see the proof of Theorem 1). Lemma 5 establishes the effectiveness of player 1's NBWT posture $\gamma_{1}^{*}$ against any relevant posture of player 2 . The following notation will be used in the proof.

Fix $z=\left(z_{1}, z_{2}\right)$ and an equilibrium of the overall game, and consider the continuation game following the choice of (arbitrary) postures $\left(\gamma_{1}, \gamma_{2}\right)$. The dependence of various functions and terms introduced below on $z$, on the equilibrium in question, and on $\left(\gamma_{1}, \gamma_{2}\right)$ is not made explicit in the notation but should be understood in what follows.

Associated with the continuation game are "distribution functions " $F_{i}(\cdot)$, $i=1,2$ where $F_{i}(\tau)$ is the probability that player $i$ reveals rationality by $\tau$ conditional upon player $j$ not revealing rationality prior to $\tau$. Note that the distribution functions and the terms defined below are specific to the equilibrium in question.

The proof proceeds by demonstrating the effectiveness of player 1's NBWT posture $\gamma_{1}^{*}$ against any relevant posture of player 2 . This is formalized in Lemma 5.

Lemma 5 Invoke Assumption 1. For any $\xi>0, R \in(0, \infty)$ and $\bar{\mu}>0$

there exists $\delta>0$ such that if $z_{i} \leq \delta, i=1,2$ and $\max \left\{\frac{z_{1}}{z_{2}}, \frac{z_{2}}{z_{1}}\right\} \leq R$, then for any perfect Bayesian equilibrium $\sigma$ the payoff to a rational player 1 in 
the continuation game $\left(\gamma_{1}^{*}, \gamma_{2}\right)$ is at least $\left(u_{1}^{*}-\xi / 2\right)$ for any $\gamma_{2} \in \Gamma_{2}$ which a rational player 2 adopts in equilibrium with probability $\mu_{2}\left(\gamma_{2}\right) \geq \bar{\mu}$.

The proof of Lemma 5 is presented in nine steps.

Step 1. Implications of Stationarity of $\gamma_{1}^{*}$

Fix a PBE $\sigma$ and a posture $\gamma_{2}$ for 2, and consider the continuation game starting from date $(0,-1)$ after 1 has adopted his NBWT posture and 2 has adopted $\gamma_{2}$. The profile $\sigma$ induces an equilibrium on that continuation game. Recall that $\eta_{i}=\frac{z_{i} \pi_{i}\left(\gamma_{i}\right)}{z_{i} \pi_{i}\left(\gamma_{i}\right)+\left(1-z_{i}\right) \mu_{i}\left(\gamma_{i}\right)}$ is the posterior probability that player $i$ who chooses $\gamma_{i}$, is behavioral.

Because of the stationarity of 1's offer and the nature of the Nash threat, a rational type of player 2 must concede with probability 1 in finite time (see Lemma 6 in the Appendix). Moreover a rational player 2 reveals rationality by, in effect, accepting 1's offer (see Lemma 7 in the Appendix). ${ }^{17}$ These "Coasean" results are closely related to Lemma 1 of Section 3, and do not hold for arbitrary non-stationary $\gamma_{1}$.

Definition $1 \tau^{*} \equiv \inf \left\{\tau \mid u_{1}^{*} \leq \phi_{1}\left(u_{2}(\tau)\right)\right.$ or $1-F_{1}(\tau)=\eta_{1}$ or $1-F_{2}(\tau)=$ $\left.\eta_{2}\right\}$

Thus $\tau^{*}$ is the first date by which (1) a rational type of either player 1 or 2 reveals rationality (i.e. does not follow $\gamma_{i}$ ) with probability 1 , or $(2)$ the demands generated by the pair of postures $\left(\gamma_{1}^{*}, \gamma_{2}\right)$ are mutually compatible.

Step 2. Concession Distribution Functions

Concession behavior strictly within rounds is driven by the familiar logic of the war of attrition, with parameters given by the constant offers and flow payoffs corresponding to the round in question. Specifically, suppose that $\tau^{\prime}, \tau^{\prime \prime}$ are dates within round $n \in \mathcal{N}$ with $\tau^{*} \succ \tau^{\prime \prime} \succ \tau^{\prime}$, and $(n+1)>$ $t\left(\tau^{\prime \prime}\right)>t\left(\tau^{\prime}\right)>n$, and that $F_{i}\left(\tau^{\prime \prime}\right)>F_{i}\left(\tau^{\prime}\right)$ for some $i=1,2$.

We first argue that $\phi_{i}\left(u_{j}(\tau)\right)>d_{i}(\tau)$ for all $\tau \in\left((n,+2), \tau^{\prime \prime}\right)$ and $i=$ 1,2 . For $i=2$ this follows from the definition of the NBWT posture $\gamma_{1}^{*}$ (and our regularity assumption which excludes the exceptional case $u_{2}^{*}=$ $\left.d_{2}\left(m_{1}^{*}, m_{2}^{*}\right)\right)$. Recall also Assumption 3: for all $\gamma_{2}$, the pair $\left(\gamma_{1}^{*}, \gamma_{2}\right)$ generates offers and flow payoffs such that $\phi_{1}\left(u_{2}(\tau)\right) \neq d_{1}(\tau)$. Finally suppose that

\footnotetext{
${ }^{17}$ Suppose that we had allowed rational players to announce something outside the set of their behavioral types, or to stay silent. For the same reasons as in Lemmas 1 and 7 , a player 2 who made one of those alternative announcements would accept 1's NBWT offer immediately, having revealed rationality.
} 
$\phi_{1}\left(u_{2}(\tau)<d_{1}(\tau)\right)$. We show that this contradicts our initial assumption that $F_{i}\left(\tau^{\prime \prime}\right)>F_{i}\left(\tau^{\prime}\right)$ for some $i=1,2$. The inequality $\phi_{1}\left(u_{2}(\tau)\right)<d_{1}(\tau)$ implies that player 1 is strictly better off conceding at the end of the round than at any date within the round, independently of 2's concession behavior. Hence $F_{1}\left(\tau^{\prime \prime}\right)=F_{1}((n,+2))$. Since $u_{2}^{*}>d_{2}(\tau)$ it follows that 2 is strictly better off conceding at $(n,+2)$ than at $\tau^{\prime \prime}$ or at any date in between. Hence $F_{2}\left(\tau^{\prime \prime}\right)=F_{2}\left(\tau^{\prime}\right)$ also, a contradiction.

For all $n \in \mathcal{N}$ let

$$
\bar{\tau}(n)=\inf \left\{\tau \mid F_{i}(\tau)=F_{i}((n+1,-2))\right\} .
$$

By the preceding argument, $\phi_{i}\left(u_{j}(t(\tau))\right)>d_{i}(t(\tau))$ for all $\tau \in((n,+2), \bar{\tau}(n))$ and $i=1,2$. Consequently, the analysis within the time interval $(n, t(\bar{\tau}(n)))$ is as in the usual war of attrition, with equilibrium behavior governed by the basic principle that a normal player delays conceding only in the expectation that the other player might concede in the interim. Thus we have the familiar result that the players concede with constant hazard rates $\lambda_{i}(s)$ for $s \in$ $(n, t(\bar{\tau}(n)))$, where

$$
\lambda_{i}(s)=r \cdot \frac{\phi_{i}\left(u_{j}((n,+2))\right)-d_{i}((n,+2))}{u_{i}((n,+2))-\phi_{i}\left(u_{j}((n,+2))\right)}
$$

Integrating this expression yields:

$$
\left(1-F_{i}(\tau)\right)=e^{-\lambda_{i}(s)(t(\tau)-n)}\left(1-F_{i}((n,+2))\right)
$$

This discussion is summarized in Lemma 8 below, where $\bar{\tau}(n)$ is defined as above.

Lemma 8 For all $\tau^{\prime}, \tau^{\prime \prime}$ and $n \in \mathcal{N}$ with $\tau^{*} \succ \tau^{\prime \prime} \succ \tau^{\prime}$, and $(n+1)>$ $t\left(\tau^{\prime \prime}\right)>t\left(\tau^{\prime}\right)>n$, if $F_{i}\left(\tau^{\prime \prime}\right)>F_{i}\left(\tau^{\prime}\right)$ for some $i=1,2$ then for $k=1,2$

$$
\left(1-F_{k}(\tau)\right)=e^{-\lambda_{k}(n)(t(\tau)-n)}\left(1-F_{k}((n,+2))\right) \text { for all } \tau \in((n,+2), \bar{\tau}(n)) .
$$

Note for later use that $\lambda_{1}(s)>\lambda_{2}(s)$ for $s \in(n, t(\bar{\tau}(n)))$, where we define

$$
\lambda_{i}(s)=\left\{\begin{array}{cc}
r \cdot \frac{\phi_{i}\left(u_{j}((n,+1))\right)-d_{i}((n,+1))}{u_{i}((n,+1))-\phi_{i}\left(u_{j}((n,+1))\right)} & \text { for } s \in(n, t(\bar{\tau}(n))) \\
0 & \text { otherwise }
\end{array}\right.
$$


The argument is exactly the same as in Lemma 3 of Section 3.

Let $\theta_{i}(\tau)$ denote the probability with which $i$ reveals rationality at $\tau$, conditional upon not having revealed rationality prior to $\tau$.

Define $P_{i}(n)$ by

$$
\left(1-P_{i}(n)\right)=\prod_{k \in\{-2,-1,+1,+2\}}\left(1-\theta_{i}((n, k))\right)
$$

Thus, $P_{i}(n)$ is the probability of player $i$ 's conceding sometime within the range $(n,-2),(n,-1),(n, 1)$ and $(n, 2)$, conditional on not having conceded before then.

An implication of Lemma 8 is that positive probability concessions can only occur at the end, between or at the beginning of rounds, but not strictly within rounds. Thus the only dates at which player $i$ might reveal rationality with strictly positive probability are those $\tau$ for which $t(\tau) \in \mathcal{N}$.

Hence,

$$
1-F_{i}(\tau)=e^{-\int_{0}^{t(\tau)} \lambda_{i}(s) d s} \prod_{\nu \leq \tau}\left(1-\theta_{i}(\nu)\right)
$$

and for $\tau$ for which $t(\tau) \notin \mathcal{N}$,

$$
\left(1-F_{i}(\tau)\right)=e^{-\int_{0}^{t(\tau)} \lambda_{i}(s) d s} \prod_{\substack{n \in \mathcal{N} \\ n \leq t(\tau)}}\left(1-P_{i}(n)\right)
$$

Step 3. Discrete Concessions by Player 2

We seek to show that after time 0 , player 1 reveals rationality faster than 2. This is the case in regions of continuous concession, for the same reasons as in Section 3. It will also be necessary to compare discrete concession probabilities by 1 and 2 .

Each discrete concession by 2 is tightly linked to a contemporaneous reduction in what 1 can extract from 2 , that is, to a "down jump" (see the preamble to the proof of Theorem 2). Lemma 9 provides an upper bound on the concession probability by 2 that can be provoked by a down jump from value $a$ to $b<a$.

Some key definitions follow. Define $v_{i}^{j}(\tau)$ as the supremum over possible (given player $j$ 's strategy) payoffs to $i$, conditional upon revealing rationality at $\tau$ (given that $i$ and $j$ have not revealed rationality prior to $\tau$ ). Recall that if $t(\tau) \notin \mathcal{N}$, the only way to reveal rationality at $\tau$ is to accept the opponent's offer. It follows that, as long as player $j$ does not accept $i$ 's offer 
at $\tau$ with strictly positive probability, $v_{i}^{j}(\tau)=\phi_{i}\left(u_{j}(\tau)\right)$. Let $v_{j}^{j}(\tau)$ denote normal player $j$ 's expected equilibrium payoff conditional upon player $i$ revealing rationality at $\tau$. It is notationally cumbersome to keep track of which player has the move at $(n,-1)$ and $(n,+1)$ respectively. In this context, extending the domain of definition of $v_{i}^{j}($.$) to include the dummy date (n, 0)$ is helpful. Thus we define $v_{i}^{j}((n, 0)) \equiv v_{i}^{j}(\tau)$ where $\tau \in\{(n,-1),(n,+1)\}$ is the date at which player $i$ may change her (offer, action) pair between the round ending at $(n,-2)$ and the round beginning at $(n,+2)$. We define $v_{j}^{j}((n, 0))$ analogously (that is, normal player $j$ 's expected equilibrium payoff conditional upon player $i$ revealing rationality at $\tau \in\{(n,-1),(n,+1)\})$.

Let $w_{1}(\tau)$ be the expected equilibrium payoff to 1 (discounted to $\tau$ ) conditional upon neither player revealing rationality prior to and including $\tau$.

The total size of the down jump at round $n$ is denoted $J_{d}(n)$ and :

$$
J_{d}(n)=\max \left\{0, \max \left\{v_{1}^{2}((n,-2)), v_{1}^{2}((n, 0))\right\}-w_{1}((n,+2))\right\}
$$

Lemma 9 Suppose $u_{1}^{*}>w_{1}((n,+2))$. Then

$$
P_{2}(n) \leq \frac{J_{d}(n)}{u_{1}^{*}-w_{1}((n,+2))}
$$
Then

Proof: Suppose by way of contradiction that $P_{2}(n)>\frac{J_{d}(n)}{u_{1}^{*}-w_{1}((n,+2))}$.

$$
\begin{aligned}
P_{2}(n) u_{1}^{*}+\left(1-P_{2}(n)\right) w_{1}((n,+2)) & =P_{2}(n)\left(u_{1}^{*}-w_{1}((n,+2))+w_{1}((n,+2))\right. \\
& >\max \left\{v_{1}^{2}((n,-2)), v_{1}^{2}\left((n, 0), w_{1}((n,+2))\right)\right\}
\end{aligned}
$$

Let $\bar{k} \in\{-2,-1,1,2\}$ satisfy $\theta_{2}((n, \bar{k}))>0$ and $\theta_{2}((n, k))=0$ for all $k=\bar{k}+1, \ldots+2$. Since $P_{2}(n)>0$ such a $\bar{k}$ exists. The preceding inequality implies that 1's payoff from conceding at $(n,+2)$ or immediately after if $\bar{k}=2$, strictly exceeds 1 's payoff from conceding at $(n, \bar{k})$ or just prior to $(n, \bar{k})$. Hence $F_{1}((n, \bar{k}))=F_{1}(\tau)$ for some $\tau \prec(n, \bar{k})$ with $t(\tau)<n$. It follows that 2's payoffs from conceding at $\tau^{\prime} \in(\tau,(n, \bar{k})]$ strictly exceed 2's payoffs from conceding at $(n, \bar{k})$ which contradicts $\theta_{2}((n, \bar{k}))>0$.

Note that Lemmas 11-13 will establish that the maintained hypothesis of Lemma 9 is indeed true for an initial range of $n$ 's which suffices for our proof. 


\section{Step 4. Subdivision of Downward Jumps}

The nonstationarity of some postures $\gamma_{2}$ may induce frequent fluctuations in 1's continuation values. The discrete concessions by 2 associated with the numerous down jumps could give 2 an insurmountable advantage in the war of attrition, unless the fluctuations induce concessions by 1 of similar or greater magnitude. Comparing the effects of up and down jumps of different sizes is difficult. It is helpful to think of subdividing down jumps, and associating with each of the smaller jumps the compensating conditional concession probability by 2 (given by the formula $P_{2}=\frac{a-b}{u_{1}^{*}-b}$ ). Fortunately the overall probability of concession by 2 so obtained satisfies the bound of Lemma 9 , as the following paragraph demonstrates.

Let $P_{2}>0$ be associated with a down jump from $u_{1} \equiv \max \left\{v_{1}^{2}((n,-2)), v_{1}^{2}((n, 0))\right\}$ to $w_{1} \equiv w_{1}((n,+2))$ (and suppose that $\left.u_{1}^{*}>w_{1}((n,+2))\right)$. By Lemma 9, $1-P_{2} \geq \frac{u_{1}^{*}-u_{1}}{u_{1}^{*}-w_{1}}$. Consider the strictly decreasing sequence $u_{1}^{l}, l=0,1, \ldots, L$ such that $u_{1}^{0}=u_{1}$ and $u_{1}^{L}=w_{1}$, and define $P_{2}^{l} \equiv \frac{u_{1}^{l-1}-u_{1}^{l}}{u_{1}^{*}-u_{1}^{l}}$. Then $1-P_{2}^{l}=\frac{u_{1}^{*}-u_{1}^{l-1}}{u_{1}^{*}-u_{1}^{l}}$. Consequently

$$
\left(1-P_{2}^{1}\right)\left(1-P_{2}^{2}\right) \cdots\left(1-P_{2}^{L}\right)=\frac{u_{1}^{*}-u_{1}}{u_{1}^{*}-w_{1}} \leq 1-P_{2}
$$

Thus a down jump may be broken up into a sequence of smaller down jumps which span the same range, and yield an overall probability of concession by 2 which weakly overestimates the actual probability of concession by 2 .

\section{Step 5. Paired Up and Down Jumps}

In general, it is possible to have multiple up and down jumps in 1's continuation value, all in a single interval of non-concession by player 2 . Comparison of the respective concession probabilities of players 1 and 2 can be extremely involved, and this is relegated to the Appendix. To provide a more accessible treatment, we limit attention here to a simple case involving two perfectly complementary jumps. Readers interested in the Appendix may find it useful to get a motivating overview by looking at Steps 5 - 7 
here before turning to the material in the Appendix concerning Section 4 .

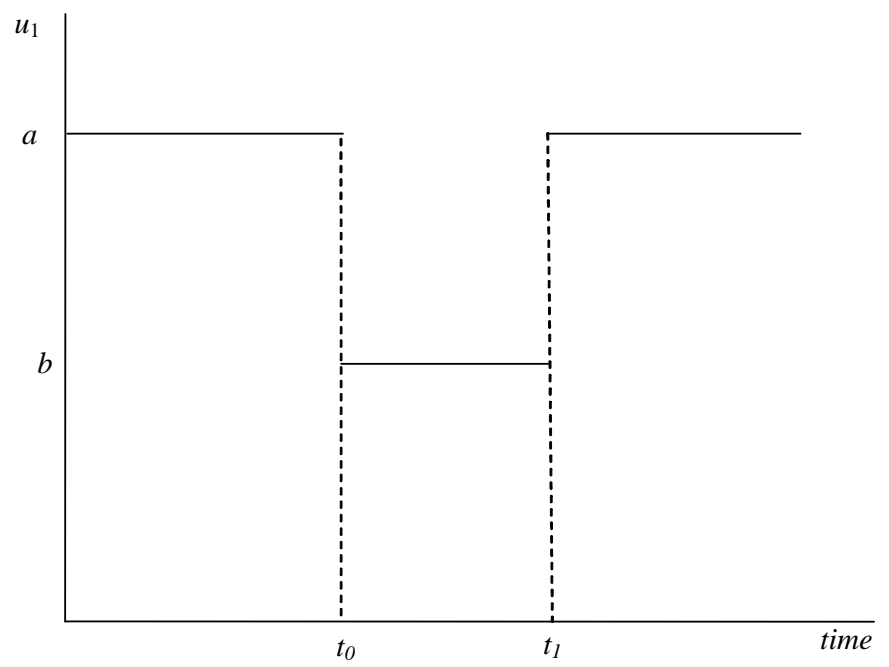

Figure 3

Figure 3 illustrates a scenario in which 1's continuation value is initially $a<u_{1}^{*}$, then falls to $b<a$, and later returns to $a$. We assume for simplicity that these continuation values coincide with what 2's posture $\gamma_{2}$ offers 1 (there are no endogenous rewards to 1 that augment what $\gamma_{2}$ offers). One can solve for the concession probability $P_{1}$ induced by the increase in value, and the concession probability $P_{2}$ induced by the earlier fall in value. By Lemma 9,

$$
P_{2} \leq \frac{a-b}{u_{1}^{*}-b}
$$

As noted earlier (in the preamble to the proof)

$$
\frac{b}{r}=\int_{t_{0}}^{t_{1}} d_{1}((s, 0)) e^{-r\left(s-t_{0}\right)} d s+e^{-r\left(t_{1}-t_{0}\right)} \frac{a}{r}
$$

or equivalently,

$$
\left(b-d_{1}\right)=e^{-r\left(t_{1}-t_{0}\right)}\left(a-d_{1}\right)
$$

where $\Delta \equiv\left(t_{1}-t_{0}\right), d_{i} \frac{\left(1-e^{-r \Delta}\right)}{r} \equiv \int_{t_{0}}^{t_{1}} d_{i}((s, 0)) e^{-r\left(s-t_{0}\right)} d s$. (Note that $d_{i}$ is the average discounted flow payoff over the interval $\left.\left(t_{0}, t_{1}\right)\right)$.

Equation (1) may be rewritten as:

$$
\left(u_{2}^{*}-d_{2}\right)\left(1-e^{-r \Delta}\right)=e^{-r \Delta} P_{1}\left(\phi_{2}(a)-u_{2}^{*}\right)
$$


where we have replaced $v_{2}^{2}(\tau)$ (the payoff to a normal player 2) with $\phi_{2}(a)$. This substitution is valid if 1 obtains $a$ by accepting an improved offer from 2 (see the last paragraph of this step).

Combining (3) and (4) yields

$$
P_{1}=\frac{u_{2}^{*}-d_{2}}{\phi_{2}(a)-u_{2}^{*}} \cdot \frac{a-b}{b-d_{1}}
$$

Hence $P_{1}>P_{2}$ if

$$
\frac{u_{2}^{*}-d_{2}}{b-d_{1}}>\frac{\phi_{2}(a)-u_{2}^{*}}{u_{1}^{*}-b}
$$

To see that this inequality does hold, note that:

1. $\left(d_{1}, d_{2}\right)$ must be on or below the line joining $d\left(m_{1}^{*}, m_{2}^{*}\right)$ and $u^{*}$

2. the slope of the latter line equals the (absolute value) of the slope of some supporting hyperplane to the set of feasible payoffs, at $u^{*}$

3 . the frontier of the feasible set is concave, and $b<a<u_{1}^{*}$.

See Figure 4.

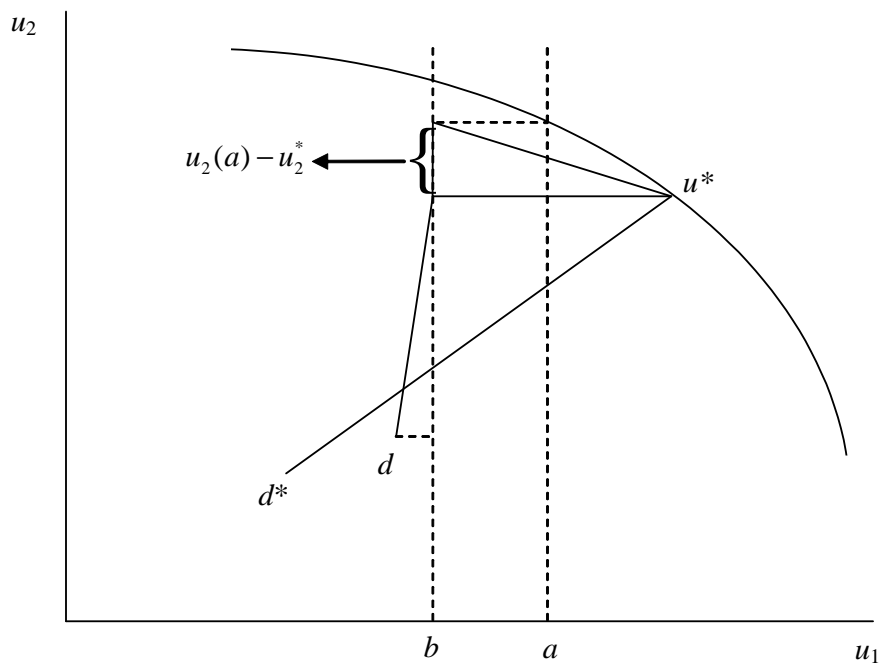

Figure 4

Thus, although the decline in 1's value from $a$ to $b$ appears to give 2 an advantage in the war of attrition (by inducing a discrete concession by 2), this advantage is outweighed by the larger discrete concession by 1 induced by the return from $b$ to $a$. Player 1's overall advantage is even greater if there are many of these paired discrete concessions, rather than the single pair illustrated here. 
What if there are more (or bigger) decreases in value than increases? For example, if value decreases from $a$ to $b$, and then stays there forever, 2 has a discrete concession not matched by a concession from 1 . This turns out to have an effect similar to 2's having a moderate reputational advantage over 1. It is swamped by other effects as the $z_{i}$ 's approach 0 . The argument in the Appendix shows that as long as all repeated down jumps are matched by (or "covered by" - see the Appendix) up jumps, 1's asymptotic advantage will be decisive. But repeated down jumps are indeed matched by up jumps: if value falls from 6 to 4, say, it can't fall through that range again until it has first risen through that range. Among the difficulties dealt with in the Appendix is the fact that where 1 has multiple concession episodes in the same interval of non-concession by 2 , the respective concession probabilities often are not uniquely defined.

We are implicitly assuming that 1 obtains the payoff $a$ by accepting an improved offer from 2 ; it might also be that 1 obtains $a$ by revealing rationality but not accepting 2 's offer. In this case the resultant equilibrium payoff to a normal 2 may be different from $\phi_{2}(a)$. This subtlety and related issues are dealt with in the Appendix.

\section{Step 6. Bounds on Equilibrium Distribution Functions.}

By Lemma 10 in the Appendix, when $t\left(\tau^{*}\right)=0$, the conclusion of Lemma 5 follows straightforwardly. Now suppose $t\left(\tau^{*}\right)>0$. Recall that $\eta_{2}(\tau)$ is the posterior probability that 2 is behavioral conditional upon 2 not revealing rationality up until and including date $\tau$. Let $\widetilde{\eta}>0$ be as defined in Lemma 12 in the Appendix. By Lemma 13 (in the Appendix) there exists $\tau \preceq \tau^{*}$ such that $\eta_{2}(\tau) \geq \widetilde{\eta}$. Let $\widetilde{\tau}=\inf \left\{\tau \mid \eta_{2}(\tau) \geq \widetilde{\eta}\right\}$. For some motivating discussion of Lemmas 11-13 see the discussion preceding the statement of the Lemmas in the Appendix. Let $\eta_{i}$ denote the posterior probability (at the start of the continuation game) that a player who adopts the posture $\gamma_{i}$ is behavioral. Then

$$
\eta_{2}(\widetilde{\tau})=\frac{\eta_{2}}{1-F_{2}(\widetilde{\tau})} \geq \widetilde{\eta}
$$

Furthermore,

$$
\eta_{1}(\widetilde{\tau})=\frac{\eta_{1}}{1-F_{1}(\widetilde{\tau})} \leq 1
$$

The goal is to establish that for small $z_{i}$ 's, the only way for the above inequalities to be satisfied is for $P_{2}(0)$ to be close to 1 . However the true distribution functions are difficult to work with. Instead we define modified functions $\widehat{F}_{i}(\tau)$ for which $\widehat{P}_{i}(0)=P_{i}(0)$ but which otherwise (weakly) underestimate 1's probability of concession and overestimate 2's. Combined with 
the earlier inequalities, this yields

$$
\begin{aligned}
\eta_{2} & \geq \widetilde{\eta}\left(1-F_{2}(\widetilde{\tau})\right) \geq \widetilde{\eta}\left(1-\widehat{F}_{2}(\widetilde{\tau})\right) \\
\text { and } \quad \eta_{1} & \leq 1-F_{1}(\widetilde{\tau}) \leq\left(1-\widehat{F}_{1}(\widetilde{\tau})\right.
\end{aligned}
$$

We show below that for small $z_{i}$ 's the above inequalities imply that $\widehat{P}_{2}(0)$ is close to 1 . That is, 2 concedes "too slowly" relative to 1 even when we overestimate 2's rate of concession and underestimate 1's.

\section{Step 7. Modified Distribution Functions.}

Recall from Step 2 that

$$
1-F_{i}(\widetilde{\tau})=e^{-\int_{0}^{t(\widetilde{\tau})} \lambda_{i}(s) d s}\left(1-P_{i}^{0}\right)\left(1-P_{i}^{1}\right) \ldots\left(1-P_{i}^{L_{i}}\right)
$$

where $l=0,1, \ldots, L_{i}$ indexes positive probability concessions by player $i$ until date $\widetilde{\tau}$.

For Player 2, any positive probability concession must be associated with a down jump (Lemma 9). Let the $l^{\text {th }}$ down jump occur at date $\tau(l)$ (assumed to be increasing in $l$ ) and entail a drop in payoff to player 1 from $a(l)$ to $b(l)$. For any $l$ such that $a(l+1)>b(l)$, Lemma 14 in the Appendix establishes the intuitively plausible result that between dates $\tau(l)$ and $\tau(l+1)$ there must exist a consecutive sequence of "shadows" corresponding to up jumps in 1's payoffs from a payoff $b \leq b(l)$ to $a \geq a(l+1)$. Down jumps over payoff drops which have also occurred at an earlier date are offset by up-jumps covering (at least) the same range (see Lemmas 15 and 16). By Steps 4 and 5, we can match such "repeated" down jumps with up jumps which span the same range. Thus one can construct new functions $\widehat{F}_{i}, i=1,2$ for which up jumps and down jumps are matched as follows:

$$
\begin{aligned}
1-\widehat{F}_{1}(\widetilde{\tau}) & =\left(1-\widehat{P}_{1}^{0}\right)\left(1-\widehat{P}_{1}^{1}\right) \ldots\left(1-\widehat{P}_{1}^{K}\right) e^{-\int_{0}^{t(\widetilde{\tau})} \lambda_{1}(s) d s} \\
1-\widehat{F}_{2}(\widetilde{\tau}) & =\left(1-\widehat{P}_{2}^{0}\right)\left(1-\widehat{P}_{2}^{1}\right) \ldots\left(1-\widehat{P}_{2}^{K}\right)\left(1-\widehat{P}_{2}^{K+1}\right) e^{-\int_{0}^{t(\widetilde{\tau})} \lambda_{2}(s) d s}
\end{aligned}
$$

where for $k=1, \ldots, K, \widehat{P}_{2}^{k}$ corresponds to a "down jump" from some $u_{1}^{k}$ to $u_{1}^{k}-\Delta$ and $\widehat{P}_{1}^{k}$ corresponds to a matched up-jump from $u_{1}^{k}-\Delta$ to $u_{1}^{k}$ between times $\underline{t}^{k}$ and $\bar{t}^{k}$ respectively. We set $\widehat{P}_{i}^{0}=P_{i}^{0}$. The latter is the probability of revealing rationality at the very start of the game and is the same for the original and modified distributions. The unmatched term $\widehat{P}_{2}^{K+1}$ accounts for the possibility of "non-repeating" down jumps. The modified distribution function $\widehat{F}_{1}$ neglects some concession episodes, since

1. it is possible that some $P_{1}^{l}>0$ are not associated with up jumps (see the remarks preceding the proof of Theorem 2), and 
2. some up jumps might not simply be "offsetting" repeated down jumps.

Of course this is consistent with underestimating 1's distribution function, and as desired we have:

$$
\left(1-\widehat{F}_{1}(\widetilde{\tau})\right) \geq\left(1-F_{1}(\widetilde{\tau})\right) .
$$

By setting $\widehat{P}_{2}^{K+1}$ "generously" we can furthermore guarantee that $(1-$ $\left.\widehat{F}_{2}(\widetilde{\tau})\right) \leq\left(1-F_{2}(\widetilde{\tau})\right)$. By Lemmas 11-13 there exists $\varepsilon>0$ such that $u_{1}^{*}-\varepsilon$ is an upper bound on player 1's expected equilibrium payoff at any $\tau \preceq \widetilde{\tau}$ for $\widetilde{\tau}$ as defined in Step 6. The highest possible $\widehat{P}_{2}^{K+1}$ is associated with an offer that drops from $u_{1}^{*}-\varepsilon$ to $\underline{u}_{1}$, the smallest payoff to 1 in the efficiency frontier of the stage game. Thus a generous specification of $\widehat{P}_{2}^{K+1}$ is

$$
\left(1-\widehat{P}_{2}^{K+1}\right)=\frac{\varepsilon}{u_{1}^{*}-\underline{u}_{1}} \equiv a_{2}>0 .
$$

By the analysis of Step 4, all non-repeating down jumps are covered by the term $\widehat{P}_{2}^{K+1}$ as defined above.

Step 8. Player 1 Concedes Faster than 2.

As noted following Lemma 8,

$$
\begin{array}{ll}
\lambda_{1}(s)>\lambda_{2}(s) & \text { for } s \in(n, t(\bar{\tau}(n))) \\
\lambda_{1}(s)=\lambda_{2}(s)=0 & \text { otherwise. }
\end{array}
$$

Can we compare $\hat{P}_{1}^{k}$ corresponding to an up jump from $\underline{w}_{1}^{k}$ to $\bar{w}_{1}^{k}$ between times $\underline{t}_{k}$ and $\bar{t}^{k}$ to $\hat{P}_{2}^{k}$ corresponding to a down jump from $\bar{w}_{1}^{k}$ to $\underline{w}_{1}^{k}$ ? Let $\hat{P}_{2}^{k}=\frac{\bar{w}_{1}^{k}-\underline{w}_{1}^{k}}{u_{1}^{*}-\underline{w}_{1}^{k}}$ as usual. As defined in the Appendix (see proof of Lemma 15), $\hat{P}_{1}^{k}$ solves

$$
\frac{u_{2}^{*}}{r}=\int_{\underline{t}_{k}}^{\bar{t}_{k}} d_{2}((s, 0)) e^{-r\left(s-\underline{t}_{k}\right)} d s+e^{-r\left(\bar{t}_{k}-\underline{t}_{k}\right)}\left[\frac{\widetilde{\phi}_{2}\left(w_{1}^{k}\right)}{r} \hat{P}_{1}^{k}+\frac{u_{2}^{*}}{r}\left(1-\hat{P}_{1}^{k}\right)\right]
$$

where $w_{1}^{k} \in\left(\underline{w}_{1}^{k}, \bar{w}_{1}^{k}\right], \widetilde{\phi}_{2}(.) \equiv \phi_{2}()+.\kappa$ and $\kappa$ is as given in Lemma 12. This yields

$$
\hat{P}_{1}^{k}=\frac{u_{2}^{*}-d_{2}}{\widetilde{\phi}_{2}\left(w_{1}^{k}\right)-u_{2}^{*}} \cdot \frac{\bar{w}_{1}^{k}-\underline{w}_{1}^{k}}{\underline{w}_{1}^{k}-d_{1}}
$$

The formulas for $\hat{P}_{1}^{k}$ correspond to those in Step 5 with a replaced by $\bar{w}_{1}^{k}$ and $b$ by $\underline{w}_{1}^{k}$. In addition $\phi_{2}\left(\bar{w}_{1}^{k}\right)$ is replaced by $\widetilde{\phi}_{2}\left(w_{1}^{k}\right)$ for some $w_{1}^{k} \in$ 
$\left(\underline{w}_{1}^{k}, \bar{w}_{1}^{k}\right]$. As in Step 5 it follows that $\hat{P}_{1}^{k}>\hat{P}_{2}^{k}$ if

$$
\frac{u_{2}^{*}-d_{2}}{\underline{w}_{1}^{k}-d_{1}}>\frac{\widetilde{\phi}_{2}\left(w_{1}^{k}\right)-u_{2}^{*}}{u_{1}^{*}-\underline{w}_{1}^{k}} .
$$

If we had $\phi_{2}\left(w_{1}^{k}\right)$ on the RHS the inequality would follow by the same reasoning (as in Step 5). However if $\kappa>0$ (recall that $\widetilde{\phi}_{2}()=.\phi_{2}()+.\kappa$ ) is chosen small enough the inequality is preserved, indeed uniformly as clarified in the next paragraph.

By Lemmas 11-13 there exists $\varepsilon>0$ such that for all $\tau \preceq \widetilde{\tau}, w_{1}^{+}(\tau) \leq$ $u_{1}^{*}-\varepsilon$ uniformly across $\left(z_{1}, z_{2}\right) \in(0,1)^{2}$ and possible equilibria. Hence $u_{1}((n,+1)) \leq u_{1}^{*}-\varepsilon$ and $\bar{w}_{1}^{k} \leq u_{1}^{*}-\varepsilon$. It follows that there exists $\alpha^{\prime}>1$ such that

$$
\lambda_{1}(s) \geq \alpha^{\prime} \lambda_{2}(s) \text { for all } s .
$$

Fixing $\varepsilon>0$ we may choose $\kappa>0$, small enough so that for some $\alpha \in\left(1, \alpha^{\prime}\right)$ we also have

$$
\left(1-\hat{P}_{1}^{k}\right) \leq\left(1-\hat{P}_{2}^{k}\right)^{\alpha} \text { for all } k=1, \ldots, K
$$

uniformly across $z_{1}, z_{2}$ and possible equilibria.

Step 9. $P_{2}(0) \cong 1$ for $z_{1}, z_{2} \cong 0$.

To complete the proof we show that when the perturbation probabilities $z_{1}, z_{2}$ are small, 2 must concede with probability close to 1 at time zero, which, as noted earlier, establishes the desired lower bound on 1's expected payoff.

Recall that

$$
\begin{aligned}
& \eta_{1} \leq 1-\widehat{F}_{1}(\widetilde{\tau})=\left(1-P_{1}^{0}\right) A_{1} \\
& \frac{\eta_{2}}{\widetilde{\eta}} \geq 1-\widehat{F}_{2}(\widetilde{\tau})=\left(1-P_{2}^{0}\right) A_{2}\left(1-\widehat{P}_{2}^{K+1}\right)
\end{aligned}
$$

where $A_{i}=e^{-\int_{0}^{t(\widetilde{\tau})} \lambda_{i}(s) d s}\left(1-\widehat{P}_{i}(1)\right) \ldots\left(1-\widehat{P}_{i}(K)\right)$, and $P_{i}(0)=\widehat{P}_{i}(0)$ is the initial probability with which player $i$ concedes.

It follows that

$$
\begin{aligned}
\eta_{1} & \leq\left(1-P_{1}(0)\right) A_{2}^{\alpha} \\
\frac{\eta_{2}}{\widetilde{\eta}\left(1-P_{2}(0)\right)\left(1-\widehat{P_{2}}(K+1)\right)} & \geq\left[\frac{\eta_{1}}{\left(1-P_{1}(0)\right)}\right]^{1 / \alpha} \\
\frac{\eta_{2}}{\eta_{1}} \cdot \eta_{2}^{\alpha-1} & \geq \frac{\left(1-P_{2}(0)\right)^{\alpha}}{\left(1-P_{1}(0)\right)} \cdot a_{2}^{\alpha}
\end{aligned}
$$


where $a_{2} \equiv \widetilde{\eta}\left(1-\hat{P}_{2}(K+1)\right)>0$ and $\alpha>1$ is as defined in Step 8 .

This analysis applies to any $z_{1}, z_{2}$. Suppose $\frac{z_{2}}{z_{1}} \leq R$ and $\mu_{2}\left(\gamma_{2} ; z_{2}\right) \geq \underline{\mu}$. Then, as shown in the proof of Lemma 4 of Section 3 ,

$$
\frac{\eta_{2}}{\eta_{1}} \leq R C
$$

for a given $R \in(0, \infty)$ and some finite constants $B, C$ independent of $\left(z_{1}, z_{2}\right)$. Returning to (5) we obtain

$$
R C\left(z_{2} B\right)^{\alpha-1} \geq \frac{\left(1-P_{2}^{0}\right)^{\alpha}}{\left(1-P_{1}^{0}\right)} \cdot a_{2}^{\alpha}
$$

Hence $P_{2}^{0} \geq 1-(R C)^{\frac{1}{\alpha}}\left(1-P_{1}^{0}\right)^{\frac{1}{\alpha}} \frac{1}{\alpha}\left(z_{2} B\right)^{\frac{\alpha-1}{\alpha}}$, which is close to 1 for $\delta>0$ small enough and $z_{2} \leq \delta$. Normal player 1's payoff is at least $P_{2}(0) u_{1}^{*}+$ $\left(1-P_{2}(0)\right) \underline{d}_{1}$, which in turn is at least $u_{1}^{*}-\frac{\xi}{2}$ for $\delta$ small enough and (consequently for) $P_{2}(0)$ close enough to 1 . (Recall that $\underline{d}_{1}$ is the lowest possible payoff to 1 in the stage game $G$ ). This completes the proof of Lemma 5 , from which Theorem 2 follows, as noted earlier.

\section{Existence of Equilibrium}

This Section establishes the existence of perfect Bayesian equilibrium (PBE) for a wide class of perturbed bargaining games. For any such game $\mathcal{G}(z)$ (defined in Section 2), we define in turn three more games, each more tractable than the last. The first simplification involves replacing $\mathcal{G}(z)$ with a concession game. From this is defined a concession game in discrete time, which is then truncated to yield one that is equivalent to a discrete, finite-horizon game. Standard arguments (Nash 1950b) guarantee that this last game has a Nash equilibrium.

It then remains to show that this equilibrium can be extended to Nash equilibrium in the games from which the simplest game was derived, and finally to a $\mathrm{PBE}$ of $\mathcal{G}(z)$. Moving back to continuous time requires some analysis of sequences of discrete-time games. Going from the concession game back to $\mathcal{G}(z)$, the game of interest, is a greater challenge. As pointed out in the preceding Section, players might, in equilibrium, reveal rationality but not accept the opponent's current offer. This leads to potentially intractably complex equilibrium behavior, and to continuation payoffs that may depend on player $i$ 's reputation at the time when $j$ reveals rationality. Nonetheless, we are able to use the relatively simple (albeit possibly 
highly nonstationary) equilibrium proved to exist in $\mathcal{C}(z)$ to construct a PBE of $\mathcal{G}(z)$ in which payoffs when $i$ reveals rationality depend on the date at which this occurs and on the initial postures (but not on reputations at that date).

Given a bargaining game $\mathcal{G}(z)$, the concession game $\mathcal{C}(z)$ associated with $\mathcal{G}(z)$ is identical with $\mathcal{G}(z)$ except that after the choice of any pair of postures $\left(\gamma_{1}, \gamma_{2}\right)$, in the subsequent game a player's only options are to stick to her initial posture or to concede. We do not allow her to adjust her demand even at integer times, with one exception: if at some date $(n,-1)$ player $i$ moves first and according to the initial postures $\left(\gamma_{1}, \gamma_{2}\right)$, player $j$ 's demand at $(n, 1)$ would be more than compatible with $i$ 's demand, then $i$ is allowed to increase her demand to make it exactly compatible. In this situation if $i$ does not increase her demand sufficiently then $j$ is allowed to increase his demand to the point of exact compatibility. In $\mathcal{C}(z)$, revealing rationality ends the game; it is free of the complications alluded to in the preceding paragraph.

We will consider discrete time versions of the concession game which we index by $\Delta \in(0,1)$ and which differ from the original (concession) game only in that players move discretely within rounds. That is, in round $n$, player 1 moves singly at times $(n+\Delta),(n+3 \Delta), \ldots$ and so on, and player 2 moves singly at $(n+2 \Delta),(n+4 \Delta), \ldots$ and so on, the alternating pattern continuing until $(n+W \Delta)$ where $W$ satisfies

$$
W \Delta<1 \leq(W+1) \Delta
$$

(The dates corresponding to these times are $(n+\Delta, 0),(n+2 \Delta, 0)$ and so on.) Denote such a game $\mathcal{C}(z, \Delta)$.

We now define truncated concession games. In a $\bar{\tau}$-truncated concession game, if play reaches date $\bar{\tau}$, both players must conform to their initial postures thereafter. Denote such a game $\mathcal{C}(z, \Delta, \bar{\tau})$. We denote by $\mathcal{C}(z, \bar{\tau})$ the continuous time game derived by truncating $\mathcal{C}(z)$ at $\bar{\tau}$.

Notice that $\mathcal{C}(z, \Delta)$ is a standard extensive form game in which:

- at an initial date, Nature chooses types of both players simultaneously

- next, players announce their postures

- subsequently, they play the concession game defined by their initial choice of postures $\left(\gamma_{1}, \gamma_{2}\right)$

A behavioral type $\gamma_{i}$ can only announce $\gamma_{i}$ and play according to $\gamma_{i}$. A normal type of player $i$ can announce any $\gamma_{i} \in \Gamma_{i}$ and moreover, may subsequently deviate from the announced $\gamma_{i}$ by accepting the opponent's current 
offer. Note finally, that when $t(\bar{\tau})<\infty$, the truncated game $C(z, \Delta, \bar{\tau})$ can be expressed as a finite extensive form game: since after $\bar{\tau}$ even normal players can only conform with their initial postures, we can make all the nodes at date $\bar{\tau}$ terminal nodes, with the appropriate specification of payoffs.

Several lemmas culminating in Lemma 23 are stated and proved in the Appendix.

Lemma 23 The concession game $\mathcal{C}(z)$ has a Nash equilibrium.

We now turn to $\mathcal{G}(z)$, the actual game of interest. First consider $\mathcal{G}(0)$, the bargaining game of complete information (that is, $z=0$ ).

Recall that

$$
\begin{aligned}
\underline{u_{i}} & =\min \left\{u_{i} \mid\left(u_{1}, u_{2}\right) \in \Pi^{*}\right\} \\
\overline{u_{i}} & =\max \left\{u_{i} \mid\left(u_{1}, u_{2}\right) \in \Pi^{*}\right\}
\end{aligned}
$$

where $\Pi^{*}$ is the set of strictly efficient and individually rational payoffs in the convex hull of feasible payoffs of the (finite) stage game, $G$.

Lemma 24 The set of perfect Bayesian equilibrium payoffs of $\mathcal{G}(0)$ is a superset of $\Pi^{*}$.

Proof. Let $\underline{m}_{i}$ be a strategy for $i$ which minimaxes player $j$. The following pair of strategies defines a PBE for any $a \in\left[\underline{u_{1}}, \overline{u_{1}}\right]$

$$
\begin{aligned}
& \text { Player } 1 \text { plays }\left(a, \underline{m}_{1}\right) \text { initially } \\
& \text { Player } 2 \text { plays }\left(\phi_{2}(a), \underline{m}_{2}\right) \text { initially }
\end{aligned}
$$

If 2 deviates to a more aggressive demand, player 1 plays $\left(\bar{u}_{1}, \underline{m}_{1}\right)$ and player 2 plays $\left(\phi_{2}\left(\bar{u}_{1}\right), \underline{m}_{2}\right)$ when it is next their turn to make offers. Conversely for player 1 . Moreover, following any deviation to an incompatible demand by a single player at the beginning of a round, the deviator immediately accepts the opponent's equilibrium offer while the player who did not deviate waits for her offer to be accepted. Subsequent single-player deviations which yield incompatible demands are responded to in the same manner. Suppose at the beginning of round $n$ both players deviate from the prescribed (demand, action) pairs as given by the rules above. Let $i$ be the player who moves at $(n,-1)$ and $j$ the player who moves at $(n,+1)$. If $j$ 's flow payoff in round $n$ does not exceed $i$ 's offer to $j$ then $j$ 's prescribed strategy is to accept $i$ 's offer at $(n,+2)$ (and at all subsequent dates in that round conditional upon the game not having terminated prior to that date) and $i$ 's strategy is to wait for her offer to be accepted. If this condition does not hold for $j$ but does for $i$ then the prescription is as above with the roles of $i$ and $j$ reversed. If both players' flow payoffs strictly exceed 
what they have been offered, let $b$ be player $i$ 's round $n$ flow payoff. Then neither player concedes in round $n$ and at the beginning of the next round prescribed behavior from that point on is as described at the beginning of the proof, with $b$ replacing $a$ and player $i$ replacing player 1 . This completes the recursive description of the strategy profile.

It may be verified that this pair of strategies defines a PBE. By an analogous argument, $\left(\phi_{1}\left(u_{2}\right), u_{2}\right)$ is a PBE payoff of $\mathcal{G}(0)$ for any $u_{2} \in$ $\left[\underline{u}_{2}, \bar{u}_{2}\right]$.

Lemma 24 yields an elementary "perfect folk theorem" for the efficiency frontier for this class of complete information bargaining games. (Compare to Busch and Wen (1995); see footnote 2.)

To construct from any $\mathrm{NE}$ of $\mathcal{C}(z)$ a PBE of $\mathcal{G}(z)$, we employ an assumption not needed for our characterization results in Sections 3 and 4 .

Definition 2 A posture $\gamma_{i} \in \Gamma_{i}$ is non-manipulable if after any history and at the beginning of any round, player $j$ is strictly better off accepting player $i$ 's current offer than adopting a strategy of waiting for a future offer under the hypothesis that player $i$ will conform with $\gamma_{i}$ forever after.

Assumption 4 (Non-Manipulability) All postures $\gamma_{i} \in \Gamma_{i} \quad i=1,2$ are non-manipulable.

After any history, a posture $\gamma_{i}$ explicitly offers the opponent $j$ a contract with a certain present discounted value for $j$. Assumption 4 rules out absurd postures that give rational $j$ an incentive to refuse the offer even when $j$ is sure $i$ is the behavioral type $\gamma_{i}$.

Theorem 3 (Existence) Let $\mathcal{G}(z)$ be a bargaining game which satisfies Assumption 4. Then $\mathcal{G}(z)$ has a perfect Bayesian equilibrium.

Theorem 3 follows directly from Lemma 25 below and our existence result for $\mathcal{C}(z)$.

Lemma 25 Given Assumption 4, if $\mathcal{C}(z)$ has a Nash equilibrium, then $\mathcal{G}(z)$ has a perfect Bayesian equilibrium.

Proof. Fix a Nash equilibrium $\sigma$ of $\mathcal{C}(z)$. Consider a strategy profile in $\mathcal{G}(z)$ such that a normal player $i$ mimics postures in $\Gamma_{i}$ with the same probability 
with which normal $i$ mimics postures in the equilibrium $\sigma$ of $\mathcal{C}(z)$, and for any pair of postures $\left(\gamma_{1}, \gamma_{2}\right)$ :

(i) at dates $(n,-1)$ and $(n,+1), n \in \mathcal{N}$, prior to which neither player has revealed rationality, players conform with their postures $\gamma_{i}$. They reveal rationality only at other dates (that is, by accepting an opponent's standing offer at that date). The only exception is in the event that the postures would lead to more than compatible demands at some first date $(n,+1)$. In this case normal player $i$ who has the move at $(n,-1)$ makes the just compatible demand $\phi_{i}\left(u_{j}((n,+1))\right)$ and chooses the action $\underline{m}_{i}$, where $\underline{m}_{i}$ is a strategy for $i$ which minimaxes $j$. If $i$ chooses some $u_{i}<\phi_{i}\left(u_{j}((n,+1))\right)$ then normal $j$ chooses $\left(\phi_{j}\left(u_{i}\right), \underline{m}_{j}\right)$. This follows the treatment of the more than compatible case in the concession game.

(ii) each player's distribution over concession times is the same as in $\sigma$.

(iii) after a deviation from property (i) by player $j$ alone, a normal $i$ plays the (demand, threat) pair $\left(\overline{u_{i}}, \underline{m}_{i}\right)$ when it is next player $i$ 's turn to make a demand. Thereafter $i$ adopts the same strategy which yields the PBE payoff pair $\left(\overline{u_{i}}, \phi_{j}\left(\overline{u_{i}}\right)\right)$ in the full information game $G(0)$. Normal player $i$ never accepts $j$ 's offer, and $j$ always accepts $i$ 's offer. If player $i$ is behavioral, by the non-manipulability assumption it is optimal for $j$ to accept $i$ 's offer at the beginning of the round following the revelation of rationality by $j$. In the out-of-equilibrium event that $j$ does not accept and the next round is reached, if rational player $i$ 's offer reveals that $i$ is rational then in the ensuing full information game it is again optimal for $j$ to accept $i$ 's equilibrium offer right away. On the other hand, if $i$ 's offer does not reveal rationality player $j$ should also accept, since acceptance is a best response to $i$ 's strategy, whether $i$ is behavioral (by the non-manipulability assumption) or normal.

(iv) if both players deviate from property (i) and their offers are not compatible, let $\left(u_{i}, m_{i}\right)$ denote player $i$ 's (offer, action) pair in the round in question.

(a) If for player $j, \phi_{j}\left(u_{i}\right)$ weakly exceeds $j$ 's flow payoff in that round (and if the symmetric statement is not true for player $i$ ), then from the next round onward players play a PBE of the continuation game which gives $j$ a payoff $\phi_{j}\left(u_{i}\right)$ and $i$ a payoff $u_{i}$. Within the round, $j$ 's strategy is to concede immediately at all dates, and $i$ does not concede at any date. If instead both players have received offers weakly exceeding their flow payoff, follow the instruction in (iii) above with $j$ set to 1 .

(b) If both players' flow payoffs strictly exceed what they have been offered, respectively, let $a$ be 1's current-round flow payoff. Assign to the next round the PBE giving 1 the payoff $a$ and 2 the payoff $\phi_{2}(a)$. In the 
current round neither player ever concedes.

It is easy to verify that the strategy profile defined above is a perfect Bayesian equilibrium of $\mathcal{G}(z)$

\section{Conclusion}

Infinitely repeated games have an extreme multiplicity of equilibria. This remains true when players can offer one another long-term contracts. We show that if a two-player repeated game with contracts is perturbed slightly by the introduction of behavioral types on each side, the players' expected discounted payoffs vary only negligibly across all perfect Bayesian equilibria. Those predicted payoffs are almost independent of the exogenous distribution of behavioral types, as long as the "Nash bargaining with threats" type is one of the behavioral types on each side.

More concisely, the folk theorem is replaced by a specific prediction. A player will do well by following the advice of Nash (1953) regarding her demand, and her action while waiting. A player gains essentially nothing by imitating a dynamic behavioral type rather than the static NBWT type. Establishing this requires arguments quite different from those in the existing literature. We introduce a hybrid discrete/continuous time model that facilitates the analysis of the war of attrition.

One would like to know how behavior in a repeated game depends on the properties of the one-shot game. How much advantage is attached to the ability to hurt an opponent? Is it important whether price or quantity, for example, is the strategic variable? Do fixed costs affect the division of surplus? Our results allow the application of "Nash bargaining with threats" to give questions of this kind a relatively simple treatment. 


\section{Appendix}

\section{Section 3: Stationary Postures}

Proof of Lemma 1. Fix a PBE $\sigma$ and postures $\left(\gamma_{1}, \gamma_{2}\right)$. Suppose w.l.o.g. that $i=1$ and $j=2$. Between rounds the only way for 2 to reveal rationality is to accept 1's standing offer in that round. Hence if $t$ is not an integer the result follows trivially. Now suppose that $t$ is an integer and furthermore that 2's turn to change his (demand, action) pair at $t$ comes after 1's . Then:

Step 1 There exists $\widetilde{T}<\infty$ such that 2 accepts 1's demand with probability 1 by $t+\widetilde{T}$ if $\gamma_{1}$ continues to be played until $t+\widetilde{T}$.

Since (1) $\phi_{2}\left(u_{1}\right)>\max _{m_{2}^{\prime}} d_{2}\left(m_{2}^{\prime}, m_{1}\right)(2)$ player 2 is impatient (3) 2's payoffs in $G$ are bounded above ( $G$ is finite), it follows that there exist $\beta>0$ and $T<\infty$ such that player 2 will accept 1's offer right away unless 2 believes that 1 will reveal rationality with probability at least $\beta$, between $t$ and $t+T$.

(To see this, let $\beta$ satisfy

$$
\beta \overline{u_{2}}+(1-\beta) \max _{m_{2}^{\prime}} d_{2}\left(m_{2}^{\prime}, m_{1}\right)<\phi_{2}\left(u_{1}\right)
$$

and $T<\infty$ satisfy

$$
\left.\beta \bar{u}_{2}+(1-\beta)\left(\left(1-e^{-r T}\right) \max _{m_{2}^{\prime}} d_{2}\left(m_{2}^{\prime}, m_{1}\right)+e^{-r T} \bar{u}_{2}\right)<\phi_{2}\left(u_{1}\right) .\right)
$$

Conditional upon player 2 not accepting 1's offer and upon 1 continuing to conform with $\gamma_{1}$ until $t+T$, a similar conclusion follows between $t+T$ and $t+2 T$, and so on. Since $\pi_{1}\left(\gamma_{1}\right)>0$, the posterior probability $\eta_{1}$ that 1 is behavioral at $t$ is strictly positive, and conditional upon conformity by 1 and non-acceptance by 2 the posterior probability that 1 is behavioral at $t+n T$ is $\frac{\eta_{1}}{(1-\beta)^{n}}$. Since it is also necessary that $\frac{\eta_{1}}{(1-\beta)^{n}} \leq 1$, this leads to contradiction for large $n$. It follows that there exists $\bar{T}<\infty$ such that player 2 accepts 1 's demand $u_{1}$ by $\bar{T}$ with probability 1 , conditional upon 1 continuing to conform with $\gamma_{1}$ between $t$ and $t+\bar{T}$. Suppose $\bar{T}$ is chosen such that the preceding statement is false for any $\widetilde{T}<\bar{T}$.

Step $2 \quad \bar{T}=0$

Suppose not. Then $\widetilde{u}_{2}, 2$ 's demand immediately prior to $t+\bar{T}$, exceeds $\phi_{2}\left(u_{1}\right)$, and there exists $\varepsilon>0$ such that 1 strictly prefers $u_{1} e^{-\varepsilon}$ to $\phi_{1}\left(\widetilde{u}_{2}\right)$. It follows that conditional upon sticking with $\gamma_{1}$ until $t+\bar{T}-\varepsilon, 1$ will continue to stick with $\gamma_{1}$ with probability 1 until $t+\bar{T}$. Therefore 2 should accept 
1 's demand $u_{1}$ with probability 1 strictly prior to $t+\bar{T}$, contradicting the definition of $\bar{T}$.

This completes the proof for the case under consideration. Finally suppose that 2 moves before 1 at integer $t$. The preceding argument implies that if 1 sticks with $\gamma_{1}$ then 2 accepts 1's offer immediately. Hence 1's payoff is at least $u_{1}$. On the other hand, by sticking with $\gamma_{2}$, player 2 can guarantee herself at least $\phi_{2}\left(u_{1}\right)$. (If 1 reveals rationality at $t$, then the preceding twostep argument with the roles of players 1 and 2 reversed, implies that 2's payoff is $u_{2}>\phi_{2}\left(u_{1}\right)$. If 1 sticks with $\gamma_{1}$, player 2 may accept 1 's offer at the beginning of round $t$.) Hence 2's payoff from the equilibrium revelation of rationality at $t$ is at least $\phi_{2}\left(u_{1}\right)$. Since $\left(u_{1}, \phi_{2}\left(u_{1}\right)\right)$ is an efficient payoff pair, the conclusion follows for this case also.

\section{Section 4: Nonstationary Postures}

Lemma 6 There exists $\tau$ with $t(\tau)<\infty$ such that $1-F_{2}(\tau)=\eta_{2}$.

Proof. By our regularity assumption, $\max _{m_{2}^{\prime}} d_{2}\left(m_{1}^{*}, m_{2}^{\prime}\right)<u_{2}^{*}$. The rest of the argument is virtually identical to Step 1 of the proof of Lemma 1.

Lemma 7 Consider equilibrium in the continuation game following the choice of postures $\left(\gamma_{1}^{*}, \gamma_{2}\right)$. Suppose that neither player has revealed rationality prior to date $\tau$, and that 2 reveals rationality at $\tau$ and 1 does not. Then the resultant equilibrium continuation payoff to normal player 1 is at least $u_{1}^{*}$.

Proof. The lemma follows directly from the proofs of Lemma 1 and Lemma 6 . Note that if $\tau=(n,-1)$ for some integer $n$, then player 1 may stick with $\gamma_{1}^{*}$ at $(n, 1)$ and the two-step argument of Lemma 1 implies that 2 will accept 1 's offer immediately at $(n, 2)$, yielding the payoff pair $\left(u_{1}^{*}, u_{2}^{*}\right)$. However, depending on the equilibrium, it is possible that normal 1 reveals rationality at $(n, 1)$ also, and in the continuation game which follows obtains more than $u_{1}^{*}$.

Recall that $\underline{d}_{i}$ is the lowest possible payoff to player $i$ in $G$, and that $\underline{u}_{i}$ and $\bar{u}_{i}$ are respectively the minimum and maximum payoffs to $i$ on the (strictly) Pareto efficient frontier of $G$.

Lemma 10 If $t\left(\tau^{*}\right)=0$ then a rational player 1's payoff is at least $\left(1-\eta_{2}\right) u_{1}^{*}+\eta_{2} \underline{d}_{1}$.

Proof. Recall from Step 1 of the proof of Lemma 5 in the text that $\tau^{*}=$ $\inf \left\{\tau \mid u_{1}^{*} \leq \phi_{1}\left(u_{2}(\tau)\right)\right.$ or $1-F_{1}(\tau)=\eta_{1}$ or $\left.1-F_{2}(\tau)=\eta_{2}\right\}$. We argue that if $t\left(\tau^{*}\right)=0$ the strategy "always conform with $\gamma_{1}^{*}$ " yields a rational player 1 a payoff which is at least $\left(1-\eta_{2}\right) u_{1}^{*}+\eta_{2} \underline{d}_{1}$. If $u_{1}^{*} \leq \phi_{1}\left(u_{2}\left(\tau^{*}\right)\right)$ then the conclusion follows directly. If $\left(1-F_{2}\left(\tau^{*}\right)\right)=\eta_{2}$ then normal 2 reveals 
rationality for sure by $\tau^{*}$. If 1 conforms with $\gamma_{1}^{*}$ the result now follows from Lemma 7. Finally, if $\left(1-F_{1}\left(\tau^{*}\right)\right)=\eta_{1}$ then, in the event of player 1 not revealing rationality at $\tau^{*}$, a rational player 2 should reveal rationality immediately thereafter. The conclusion again follows directly.

The faster rate of concession by 1 (both continuous and lumpy) is driven by the GAP between what 1 can extract from 2 by revealing rationality (or by conceding to 2's current demand) and 1's "reasonable" demand $u_{1}^{*}$. If the gap goes to zero then the difference in the rates goes to zero also and 1 no longer "wins" the "race" by an overwhelming margin and the argument that 2 needs to give in at the start with probability close to 1 breaks down. The next lemma establishes that this gap (which is datedependent) is uniformly bounded above zero, until 2's posterior probability of being behavioral reaches a threshold $\tilde{\eta}$.

Let $w_{1}^{+}(\tau)$ be the expected equilibrium payoff to normal 1 at $\tau$ conditional upon neither player revealing rationality strictly prior to $\tau$.

Lemma 11 For all $\gamma_{2} \in \Gamma$, there exist $\varkappa \in(0,1)$ and $\varepsilon>0$ such that $\varkappa_{1}+(1-\varkappa) u_{1}^{*}>u_{1}^{*}-\varepsilon$ and such that for all $\left(z_{1}, z_{2}\right) \in(0,1)^{2}$ and for any perfect Bayesian equilibrium of $\mathcal{G}\left(z_{1}, z_{2}\right)$ it is the case that in the continuation game following the choice of postures $\left(\gamma_{1}^{*}, \gamma_{2}\right)$, either (1) $t\left(\tau^{*}\right)=0$ or $(2)$ for all $\tau \preceq \tau^{*}$ if $\eta_{2}(\tau) \leq \varkappa$ then $w_{1}^{+}(\tau) \leq u_{1}^{*}-\varepsilon$.

Proof. Suppose $t\left(\tau^{*}\right)>0$. Since $\gamma_{1}^{*}$ and $\gamma_{2}$ have a finite number of states, there exists $\varepsilon_{1}>0$ such that $\phi_{1}\left(u_{2}(\tau)\right)<u_{1}^{*}-\varepsilon_{1}$ for all $\tau$ such that $u_{2}(\tau)>u_{2}^{*}$ (equivalently $\left.\phi_{1}\left(u_{2}(\tau)\right)<u_{1}^{*}\right)$. Clearly there exist $0<\varepsilon_{2}<\varepsilon_{1}$ and $\Delta>0$ such that $u_{1}^{*}-\varepsilon_{1}<e^{-r \Delta}\left(u_{1}^{*}-\varepsilon_{2}\right)+\left(1-e^{-r \Delta}\right) \underline{d}_{1}$, where $\underline{d}_{1}$ is the lowest possible payoff to $1 \mathrm{in} G$. (The r.h.s. of the preceding inequality is the payoff to 1 if 1 waits for time $\Delta$ to receive $\left(u_{1}^{*}-\varepsilon_{2}\right)$ while receiving the lowest possible flow payoff in the interim.) It follows that if at some $\tau$, $w_{1}^{+}(\tau) \geq u_{1}^{*}-\varepsilon_{2}$ for the first time, then player 1 will reveal rationality with probability zero for an interval of time $\Delta>0$ prior to $\tau$. (Note that we can choose $\Delta>0$ such that $\Delta<t(\tau)$.)

For normal 2 not to concede with probability 1 for $\Delta$ units of time prior to $\tau$, it must be the case that $u_{2}^{*} \leq e^{-r \Delta} u_{2}+\left(1-e^{-r \Delta}\right) d_{2}$, where $u_{2}$ is normal 2's expected equilibrium payoff at $\tau$ and $d_{2}$ is 2's (discounted average) flow payoff in the interim. By the definition of $\gamma_{1}^{*}$ and $m_{1}^{*}, 2$ 's payoff in any round must be less than or equal to $u_{2}^{*}$, and by our regularity assumption must, in fact, be strictly less. Since each posture has a finite number of states there exists $a>0$ such that $d_{2}<u_{2}^{*}-a$. Hence $u_{2} \geq$ $u_{2}^{*}+b$ for some $b>0$. It follows that if $w_{1}^{+}(\tau) \geq u_{1}^{*}-\varepsilon_{2}$, then conditional upon 2 being normal, 1's expected payoff at $\tau$ is at most $u_{1}^{*}-\varepsilon_{3}$ for some $\varepsilon_{3}>0$. Consequently $w_{1}^{+}(\tau) \leq \eta_{2}(\tau) \bar{u}_{1}+\left(1-\eta_{2}(\tau)\right)\left(u_{1}^{*}-\varepsilon_{3}\right)$. Let $\varepsilon=$ 
$\min \left\{\varepsilon_{2}, \frac{\varepsilon_{3}}{2}\right\}$. Clearly there exists $\varkappa \in(0,1)$ such that $\varkappa_{1}+(1-\varkappa) u_{1}^{*}>$ $u_{1}^{*}-\varepsilon$ and $\eta_{2}(\tau) \bar{u}_{1}+\left(1-\eta_{2}(\tau)\right)\left(u_{1}^{*}-\varepsilon_{3}\right)<u_{1}^{*}-\varepsilon$ for all $\eta_{2}(\tau) \leq \varkappa$. (Set $\varkappa=\frac{1}{2} \min \left\{\frac{\varepsilon}{u_{1}^{*}-\underline{u}_{1}}, \frac{\varepsilon}{\bar{u}_{1}-u_{1}^{*}+2 \varepsilon}\right\}$ ). The result now follows for $\varepsilon$ and $\varkappa$ so defined.

The argument comparing the rates of concession by 1 and 2 respectively also requires a tight connection between the payoff from revealing rationality to player 1 and the corresponding payoff to a normal player 2. When the posterior probabilty of a behavioral player 2 is "large" (above $\tilde{\eta}$ ) it is possible both that normal 1 can obtain a payoff $v_{1}^{2}(\tau)$ in excess of $u_{1}^{*}$ and that normal 2 obtain a payoff in excess of $\phi_{2}\left(v_{1}^{2}(\tau)\right)$.

Lemma 12 Let $\varkappa$ be defined as in Lemma 11. For any $\kappa>0$ there exists $\tilde{\eta} \in(0, \varkappa)$ such that for all $\left(z_{1}, z_{2}\right) \in(0,1)^{2}$ and for any perfect Bayesian equilibrium of $\mathcal{G}\left(z_{1}, z_{2}\right)$ it is the case, in the continuation game following the choice of $\left(\gamma_{1}^{*}, \gamma_{2}\right)$, that for all $\tau \preceq \tau^{*}$ if $\eta_{2}(\tau) \leq \tilde{\eta}$ then $v_{2}^{2}(\tau) \leq$ $\phi_{2}\left(v_{1}^{2}(\tau)\right)+\kappa$.

Proof. Proof. Let $X$ be 1's expected payoff if player 2 is behavioral and $x$ be 1's expected payoff if player 2 is normal. By definition, $v_{1}^{2}(\tau)=$ $\eta_{2}(\tau) X+\left(1-\eta_{2}(\tau)\right) x$. Then $v_{1}^{2}(\tau) \leq \eta_{2}(\tau) \bar{u}_{1}+\left(1-\eta_{2}(\tau)\right) x$. Hence

$$
x \geq \frac{v_{1}^{2}(\tau)-\eta_{2}(\tau) \bar{u}_{1}}{1-\eta_{2}(\tau)} .
$$

Consequently $v_{2}^{2}(\tau) \leq \phi_{2}(x) \leq \phi_{2}\left(\frac{v_{1}^{2}(\tau)-\eta_{2}(\tau) \bar{u}_{1}}{1-\eta_{2}(\tau)}\right)$.

It follows that for any $\kappa>0$, there exists $\tilde{\eta}$ strictly positive and small enough such that if $\eta_{2}(\tau) \leq \tilde{\eta}$, then $v_{2}^{2}(\tau) \leq \phi_{2}\left(v_{1}^{2}(\tau)\right)+\kappa$, as required.

Definition $3 \widetilde{\phi}_{2}(.) \equiv \phi_{2}()+.\kappa$.

The function $\widetilde{\phi}_{2}($.$) appears in Step 8$ of the proof of Lemma 5 in the text and in Lemma 15 below.

Lemma 13 For any equilibrium consider the continuation game following the choice of $\left(\gamma_{1}^{*}, \gamma_{2}\right)$. Let $\tilde{\eta}$ be defined as in Lemma 12. Then either $t\left(\tau^{*}\right)=0$ or $\eta_{2}\left(\tau^{*}\right) \geq \tilde{\eta}$.

Proof. Suppose $t\left(\tau^{*}\right)>0$ and $\eta_{2}\left(\tau^{*}\right)<\tilde{\eta}$. Then $1-F_{2}\left(\tau^{*}\right)>\eta_{2}$. (If $1-F_{2}\left(\tau^{*}\right)=\eta_{2}$ then $\left.\eta_{2}\left(\tau^{*}\right)=1>\tilde{\eta}\right)$. Also, by Lemma 11, $w_{1}^{+}(\tau)<u_{1}^{*}-\varepsilon$ for all $\tau \preceq \tau^{*}$ (since $\eta_{2}(\tau) \leq \eta_{2}\left(\tau^{*}\right) \leq \tilde{\eta}$ for all $\tau \preceq \tau^{*}$ ). From the definition of $\tau^{*}$ it therefore follows that $1-F_{1}\left(\tau^{*}\right)=\eta_{1}$. Consequently normal player 
2 must reveal rationality/concede immediately after $\tau^{*}$. Hence, $w_{1}^{+}\left(\tau^{*}\right) \geq$ $\tilde{\eta} \underline{u}_{1}+(1-\tilde{\eta}) u_{1}^{*}>u_{1}^{*}-\varepsilon$, which contradicts Lemma 11 .

The discussion below elaborates elements of Steps 5 and 7 in the text, in particular the discussion of repeated down jumps. As in the text, consider the $l^{\text {th }}$ down jump and suppose that player 1's payoff $b(l)$ after the $l^{\text {th }}$ down jump is strictly less than 1's payoff $a(l+1)$ at the "start" of the $(l+1)^{\text {th }}$ down jump. Between these down jumps we wish to argue that there are offsetting up jumps.

Recall from Step 5 of the proof of Theorem 2 that there is a formula for the conditional concession probability by player 1 that is needed to compensate player 2 for waiting while player 1 waits for an upward jump of a given size in 1's value. Call this the canonical formula. There are complicated cases in which this formula does not apply directly. For example, suppose that an increase in value from $b$ to $a$ at some time $\tau_{2}$ casts a shadow over the interval $\left[\tau_{0}, \tau_{2}\right]$. There might be some date $\tau_{3} \in\left(\tau_{0}, \tau_{2}\right)$ at which the continuation equilibrium rewards 1 for revealing rationality (but not conceding) just enough so that he is indifferent between doing so or waiting until $\tau_{2}$. His indifference means that there are many combinations of concession probabilities at $\tau_{1}$ and $\tau_{2}$ by 1 that are compatible with maximizing his utility, and which exactly compensate 2 for her wait from $\tau_{0}$ to $\tau_{2}$. In such cases one cannot use the canonical formula to associate with the jumps at $\tau_{2}$, a particular concession probability by 1 . Various other possible complications must also be addressed, as will become evident in the proof of Lemma 15 .

Because of the indeterminacy just described, it is important to look at the interval $\left[\tau_{0}, \tau_{2}\right]$ as a whole, rather than at the concession episodes at $\tau_{1}$ and $\tau_{2}$ separately (and hence the introduction of Definition 4).

Definition 4 The interval $\mathcal{I}$ is an interval of zero concession by player 2 if for all $\tau^{\prime}, \tau^{\prime \prime} \in \mathcal{I}, F_{2}\left(\tau^{\prime}\right)=F_{2}\left(\tau^{\prime \prime}\right)$. Such an interval is a maximal interval of zero concession by player 2 , if for all $\mathcal{I}^{+} \supseteq \mathcal{I}, \mathcal{I}^{+} \neq \mathcal{I}$ there exist $\tau^{\prime}, \tau^{\prime \prime} \in \mathcal{I}^{+}$ such that $F_{2}\left(\tau^{\prime \prime}\right)>F_{2}\left(\tau^{\prime}\right)$.

Lemma 14 asserts that between any two episodes in which 1's value falls over a certain range, say from 20 to 14 , there must be a sequence (called a "spanning sequence" - see Definition 5 following Lemma 14) of (weakly overlapping) up jumps whose union covers the interval $[14,20]$. For example, if the value falls from 22 to 13, it might later fall from 20 to 14, but before doing so it would have to somehow rise to at least 20. Associated with these up jumps are corresponding intervals of zero concession by 2 .

Lemma 14 Suppose for some $n^{\prime}, n^{\prime \prime} \in \mathcal{N}$ such that $n^{\prime}<n^{\prime \prime}$, (i) $P_{2}(n)=0$ for all $n \in \mathcal{N}, n^{\prime}<n<n^{\prime \prime}$ 
(ii) $P_{2}\left(n^{\prime}\right), P_{2}\left(n^{\prime \prime}\right)>0$ and

(iii) $w_{1}\left(\left(n^{\prime},+2\right)\right)<\max \left\{v_{1}^{2}\left(\left(n^{\prime \prime},-2\right)\right), v_{1}^{2}\left(\left(n^{\prime \prime}, 0\right)\right)\right\}$.

Then there exists a sequence of maximal intervals of zero concession by 2, $\mathcal{I}(q), q=1, \ldots, Q$, with associated left and right end-points $\underline{\tau}(q) \equiv \inf \mathcal{I}(q)$ and $\bar{\tau}(q) \equiv \sup \mathcal{I}(q)$ respectively, such that

$$
\begin{aligned}
w_{1}(\underline{\tau}(1)) & \leq w_{1}\left(\left(n^{\prime},+2\right)\right), w_{1}(\bar{\tau}(Q)) \geq \max \left\{v_{1}^{2}\left(\left(n^{\prime \prime},-2\right)\right), v_{1}^{2}\left(\left(n^{\prime \prime}, 0\right)\right)\right\} \\
w_{1}(\underline{\tau}(q+1)) & \leq w_{1}(\bar{\tau}(q)) \quad q=1, \ldots, Q-1 \text { and } \\
w_{1}(\underline{\tau}(q)) & <w_{1}(\bar{\tau}(q)) \quad q=1, \ldots, Q \\
\left(n^{\prime},+2\right) & \preccurlyeq \underline{\tau}(1) \prec \bar{\tau}(Q) \preccurlyeq\left(n^{\prime \prime},+2\right), \bar{\tau}(q) \prec \underline{\tau}(q+1), q=1, \ldots, Q(8)
\end{aligned}
$$

Proof. Since $w_{1}\left(\left(n^{\prime},+2\right)\right)<\max \left\{v_{1}^{2}\left(\left(n^{\prime \prime},-2\right)\right), v_{1}^{2}\left(\left(n^{\prime \prime}, 0\right)\right)\right\}$, there must exist a first date $\widehat{\tau} \succ\left(n^{\prime},+2\right)$ at which $v_{1}^{2}(\widehat{\tau})>w_{1}\left(\left(n^{\prime},+2\right)\right)$. It follows that player 1 does not concede in an interval immediately prior to $\widehat{\tau}$. Hence neither does 2 in an interval prior to $\widehat{\tau}$. We now argue that $\theta_{2}(\widehat{\tau})=0$ (recall that $\theta_{2}(\widehat{\tau})$ is the conditional probability with which 2 concedes at date $\left.\widehat{\tau}\right)$. Clearly $\widehat{\tau}=(n, k)$ for integer $n$ and $k \in\{-1,+1,+2\}$. Unless $k=2$ the result follows by definition. Now suppose $k=2$ and $\theta_{2}(\widehat{\tau})>0$. Then for the usual reasons $\theta_{1}(\widehat{\tau})=0$ (if not, both players would strictly prefer to delay conceding momentarily). Given that $\theta_{1}(\widehat{\tau})=0$ and that 1 does not concede in an interval immediately prior to $\widehat{\tau}$, player 2 should strictly prefer to concede prior to $\widehat{\tau}$, a contradiction. It follows that there exists a maximal interval $\mathcal{I}$ (of zero concession by 2 ) with associated left and right end-points $\underline{\tau}$ and $\bar{\tau}$ respectively, containing $\widehat{\tau}$, such that $w_{1}(\underline{\tau}) \leq w_{1}\left(\left(n^{\prime},+2\right)\right)$. It is, however, possible that $w_{1}(\bar{\tau})<w_{1}\left(\left(n^{\prime},+2\right)\right)$. In this case, $t(\bar{\tau})<n^{\prime \prime}$ and we can repeat the preceding argument replacing the starting date $\left(n^{\prime},+2\right)$ for the preceding argument, with $\bar{\tau}$. (That is, we look for the first date $\widehat{\tau} \succ \bar{\tau}$ at which $v_{1}^{2}(\widehat{\tau})>w_{1}(\bar{\tau})$, and so on.) Proceeding in this manner we obtain a first maximal interval $(\underline{\tau}(1), \bar{\tau}(1))$ for which $w_{1}(\underline{\tau}(1)) \leq w_{1}\left(\left(n^{\prime},+2\right)\right)$ and $w_{1}(\underline{\tau}(1))<w_{1}(\bar{\tau}(1))$. If $w_{1}(\bar{\tau}(1))<\max \left\{v_{1}^{2}\left(\left(n^{\prime \prime},-2\right)\right), v_{1}^{2}\left(\left(n^{\prime \prime}, 0\right)\right)\right\}, \bar{\tau}(1)$ now plays the role of $\left(n^{\prime},+2\right)$ in the initial argument. And so on, until the required sequence is obtained. Since $P_{2}\left(n^{\prime \prime}\right)>0, t(\bar{\tau}(q)) \leq n^{\prime \prime}$ for all $q$.

Definition 5 A sequence as specified in Lemma 14 is said to span $[b, a]$, where $b=w_{1}\left(\left(n^{\prime},+2\right)\right)$ and $a=\max \left\{v_{1}^{2}\left(\left(n^{\prime \prime},-2\right)\right), v_{1}^{2}\left(\left(n^{\prime \prime}, 0\right)\right)\right\}$.

By our regularity assumption regarding generic type sets (Assumption 3), $\phi_{1}\left(u_{2}(\tau)\right) \neq d_{1}(\tau)$ for all $\tau \preccurlyeq \tau^{*}$. It follows that, if within a round 
$n$ there is zero concession by player 2 , conceding at $(n,+2)$ strictly dominates conceding at any subsequent date within round $n$ for player 1 if $\phi_{1}\left(u_{2}(\tau)\right)>d_{1}(\tau)$, while if the opposite strict inequality is satisfied, conceding at $((n+1),-2)$ strictly dominates conceding at a prior date within the round. Hence within an interval such as $\mathcal{I}(q)$, player 1 reveals rationality or concedes only at the beginning, in between, or at the end of rounds contained within $\mathcal{I}(q)$.

For a sequence of maximal intervals as in Lemma 14 and Definition 5, let $x=1, \ldots, X$ index the finite set of instances at which 1 concedes at a date in $\mathcal{I}(q)$ for some $q \in\{1, \ldots, Q\}$ or at $\bar{\tau}(Q)$. (It is possible that $\bar{\tau}(Q) \notin I(Q)$; however if 1 concedes with positive probabilty at $\bar{\tau}(Q)$ our proof requires that we keep track of this.) Let $P_{1}^{x}$ denote the corresponding conditional probability of concession by 1 .

Lemma 15 translates the probabilities $P_{1}^{x}$ just defined, into modified probabilities $\hat{P}_{1}^{1}, \ldots, \hat{P}_{1}^{Y}$ such that (i) the overall probability of concession by 1 is weakly lower according to the modified probabilities than the true probabilities, and (ii) the modified probabilities are less than or equal to the numbers one would obtain by applying the canonical formula (see Step 5 in the text) to the respective up jumps in 1's value that occur in the maximal interval in question. Property (ii) is useful because if a probability $P_{1}$ is obtained by applying the canonical formula to an up jump, it can be compared easily (see Step 5) to the concession probability by 2 associated with a down jump over the same interval. Both (i) and (ii) are consistent with our need to underestimate 1's concession probabilities (see Step 6).

Lemma 15 Consider a sequence of maximal intervals of zero concession by 2 which span $[b, a]$ and suppose that $\eta_{2}(\bar{\tau}(Q)) \leq \widetilde{\eta}$, where $\widetilde{\eta}$ is as defined in Lemma 12 and $\bar{\tau}(Q)$ is as defined in Lemma 14. Let $P_{1}^{1}, \ldots, P_{1}^{X}$ be a sequence of (conditional) probabilities as specified above. Then there exist a sequence of probabilities $\hat{P}_{1}^{1}, \ldots, \hat{P}_{1}^{Y}$ and a corresponding sequence of values and dates $\underline{w}_{1}^{y}, \bar{w}_{1}^{y}, w_{1}^{y} \in\left(\underline{w}_{1}^{y}, \bar{w}_{1}^{y}\right], \underline{t}_{y}$ and, $\bar{t}_{y}, y=1, \ldots, Y$, such that:

$$
\begin{aligned}
\underline{w}_{1}^{y} & <\bar{w}_{1}^{y} \\
\underline{w}_{1}^{y} & <\underline{w}_{1}^{y+1} \leq \bar{w}_{1}^{y} \\
\bar{w}_{1}^{y} & <\bar{w}_{1}^{y+1} \\
\underline{t}_{y} & \leq \bar{t}_{y} \\
\underline{w}_{1}^{1} & \leq b, \quad \bar{w}_{1}^{Y} \geq a
\end{aligned}
$$


where the $\hat{P}_{1}^{y \prime}$ s solve the "canonical" equation

$$
\frac{u_{2}^{*}}{r}=\int_{\underline{t}_{y}}^{\bar{t}_{y}} e^{-r\left(s-\underline{t}_{y}\right)} d_{2}((s, 0)) d s+e^{-r\left(\bar{t}_{y}-\underline{t}_{y}\right)}\left[\frac{\tilde{\phi}_{2}\left(w_{1}^{y}\right)}{r} \hat{P}_{1}^{y}+\frac{u_{2}^{*}}{r}\left(1-\hat{P}_{1}^{y}\right)\right]
$$

and

$$
\left(1-P_{1}^{1}\right) \ldots\left(1-P_{1}^{X}\right) \leq\left(1-\hat{P}_{1}^{1}\right) \ldots\left(1-\hat{P}_{1}^{Y}\right)
$$

Proof. Let $\mathcal{I}$ be a maximal interval as defined above and let $\underline{\tau}$ and $\bar{\tau}$, respectively be the left and right end points of the interval. Let $\tau_{1}, \ldots, \tau_{L}$ be the finite set of dates (in ascending order) at which 1 reveals rationality within $\mathcal{I} \cup\{\bar{\tau}\}$ with corresponding conditional probabilities $P_{1}^{l}, l=1, \ldots, L$. Define

$$
\begin{aligned}
\tau_{0} & \equiv\left\{\begin{array}{cc}
(t(\underline{\tau}),+2) & \text { if } t(\underline{\tau}) \in \mathcal{N} \\
\underline{\tau} & \text { otherwise }
\end{array}\right. \\
t_{l} & \equiv t\left(\tau_{l}\right) .
\end{aligned}
$$

Let $w_{2}^{l}$ be normal player 2's expected (average discounted) payoff at date $\tau_{l}$, conditional upon player 1 not having revealed rationality until $\tau_{l}$ (inclusive). Then

$$
\frac{w_{2}^{l}}{r}=\left[\int_{t_{l}}^{t_{l+1}} d_{2}((s, 0)) e^{-r\left(s-t_{l}\right)} d s\right]+e^{-r\left(t_{l+1}-t_{l}\right)}\left[\frac{v_{2}^{2}\left(\tau_{l}\right)}{r} P_{1}^{l+1}+\frac{w_{2}^{l+1}}{r}\left(1-P_{1}^{l+1}\right)\right]
$$

where $v_{2}^{2}(\tau)$ denotes the expected equilibrium payoff to normal player 2 , conditional upon 1 revealing rationality at $\tau$.

We will define a new sequence $\hat{\tau}_{0}, \ldots, \hat{\tau}_{K}$ and corresponding sequence of probabilities $\hat{P}_{1}^{1}, \ldots, \hat{P}_{1}^{K}$ such that

$$
\left(1-P_{1}^{1}\right) \cdots\left(1-P_{1}^{L}\right) \leq\left(1-\hat{P}_{1}^{1}\right) \cdots\left(1-\hat{P}_{1}^{K}\right)
$$

where $\hat{P}_{1}^{k}$ corresponds to an up-jump from $\underline{w}_{1}^{k}$ to $\bar{w}_{1}^{k}$ which can be matched with a down-jump from $\bar{w}_{1}^{k}$ to $\underline{w}_{1}^{k}$. Furthermore, $\underline{w}_{1}^{1} \leq w_{1}^{1}, \underline{w}_{1}^{k}<\underline{w}_{1}^{k+1} \leq \bar{w}_{1}^{k}$, and $\bar{w}_{1}^{K}=w_{1}^{L}$.

The argument proceeds by modifying the original $P_{1}^{l}$ 's in successive steps such that the modified $P_{1}^{l}$ 's (denote those in generic step $s^{\prime}$ by $P_{1}^{l}\left(s^{\prime}\right)$ ) yield a product $\left(1-P_{1}^{1}\left(s^{\prime}\right)\right) \cdots\left(1-P_{1}^{L}\left(s^{\prime}\right)\right)$ which is weakly higher than the corresponding product from the preceding step. 
Within any maximal interval of zero concession (by 2), we wish to assign concession probabilities by 1 in the most conservative way, so that our modified concession distribution function $\widehat{F}_{1}$, will underestimate 1 's probability of conceding by any date, as desired. The following procedure achieves this, using basic properties of the incentive constraints of both players. Begin by defining a sequence

$$
\begin{aligned}
& q(0)=0, \text { and for } k=1, \ldots, K \\
& q(k)=\max \left\{\begin{array}{l}
l \mid \begin{array}{c}
v_{1}^{2}\left(\tau_{l}\right) \leq v_{1}^{2}(\tau), \\
\tau=\tau_{q(k-1)+1}, \ldots, \tau_{l} .
\end{array}
\end{array}\right\}
\end{aligned}
$$

The new sequence terminates at $K$ such that $q(K)=L$. Observe that $v_{1}^{2}\left(\tau_{q(k)}\right)<v_{1}^{2}(\tau)$ for all $\tau \succ \tau_{q(k)}$. Consequently $v_{1}^{2}\left(\tau_{q(k)}\right)$ is strictly increasing in $k$.

Define

$$
k^{*}(l)=\min \left\{\tau_{q(k)} \mid q(k) \geq l\right\}
$$

Let

$$
\begin{array}{rll}
P_{1}^{l}(0) & =P_{1}^{l} \quad l=1, \ldots, L \\
\text { and } w_{2}^{l}(0) & =w_{2}^{l} \quad l=0,1, \ldots, L
\end{array}
$$

We seek to define $P_{2}^{l}(1)$ and $w_{2}^{l}(1)$ inductively, starting with $l=L$ and moving backwards to $l=0$ (or 1 as the case may be). Recall that $s^{\prime}$ in $P_{2}^{l}\left(s^{\prime}\right)$ refers to the $s^{\prime}$-th step in modifying the initial $P_{1}^{l}$ 's. Each step itself involves an inductive definition starting with $l=L$ and moving backwards to $l=1$.

We first argue that $w_{2}^{L}(0) \leq u_{2}^{*}$. This argument involves considering a number of different cases.

a. Suppose $t(\bar{\tau}) \notin \mathcal{N}$. Then by standard arguments $\theta_{1}(\bar{\tau})=\theta_{2}(\bar{\tau})=0$. By definition of $\bar{\tau}$, conceding immediately after $\bar{\tau}$ is in the support of normal 2's equilibrium strategy $\left(F_{2}(\tau)>F_{2}(\bar{\tau})\right.$ for all $\left.\tau \succ \bar{\tau}\right)$. It follows that $w_{2}^{L} \leq u_{2}^{*}$ with strict inequality if $\tau_{L} \prec \bar{\tau}$ (recall that against $\gamma_{1}^{*}, 2$ 's flow payoff is always strictly less than $u_{2}^{*}$ ).

When $t(\bar{\tau})=n \in \mathcal{N}$ there are many sub-cases to consider.

b. $\bar{\tau}=(n,+2)$. For the usual reasons if $\theta_{i}((n,+2))>0$ then $\theta_{j}((n,+2))=$ 0 . Clearly if $\theta_{2}(\bar{\tau})>0$ then $\tau_{L} \prec \bar{\tau}$ and the result follows. If, on the other hand, $\theta_{1}(\bar{\tau})>0$ then $\tau_{L}=\bar{\tau}$ and the rest of the argument is as in a. (Conceding immediately after $\bar{\tau}$ is in the support of 2's 
equilibrium strategy). Finally, if $\theta_{1}(\bar{\tau})=\theta_{2}(\bar{\tau})=0$ the argument is as in $\mathbf{a}$.

c. $\bar{\tau}=(n,-1)$. By our definition of maximal intervals and $\bar{\tau}, \theta_{2}((n,-1))>$ 0 when 2 has the move at $(n,-1)$ and conversely $\theta_{2}((n,+1))>0$ when 2 has the move at $(n,+1)$. In the former case $\tau_{L} \prec(n,-1)$, and in the latter $\tau_{L} \prec(n,+1)$. Now Lemma 7 yields the desired conclusion.

d. $\bar{\tau}=(n,-1)$. Now we must have $\theta_{2}((n,+2))>0$ when 2 has the move at $(n,-1)$ and either $\theta_{2}((n,+1))>0$ or $\theta_{2}((n,+1))=0$ and $\left.\theta_{2}((n,+2))\right)>0$ when 2 has the move at $(n,+1)$. The case $\theta_{2}((n,+1))>$ 0 is dealt with in $\mathbf{c}$ above and $\theta_{2}((n,+2))>0$ in $\mathbf{b}$ above.

e. $\bar{\tau}=(n,-2)$. Now we must have $\theta_{2}((n,-2))>0$ unless 2 has the move at $(n,-1)$. In this case it is possible that $\theta_{2}((n,-2))=0$ and $\theta_{2}((n,-1))>0$, when the conclusion follows as in $\mathbf{c}$ above. If $\theta_{2}((n,-2))>0$ and $\theta_{1}((n,-2))=0$ then the result follows from Lemma 7 (and $\tau_{L} \prec \bar{\tau}$ ).

Finally we are left with the case $\theta_{2}((n,-2))>0$ and $\theta_{1}((n,-2))>0$. The payoffs to player 2 at $(n,-2)$ are summarized in the table below, where $\mathrm{C}$ stands for concede, $\mathrm{NC}$ for not concede and $u_{2}((n,-2))$ is 2 's standing demand at $(n,-2)$.

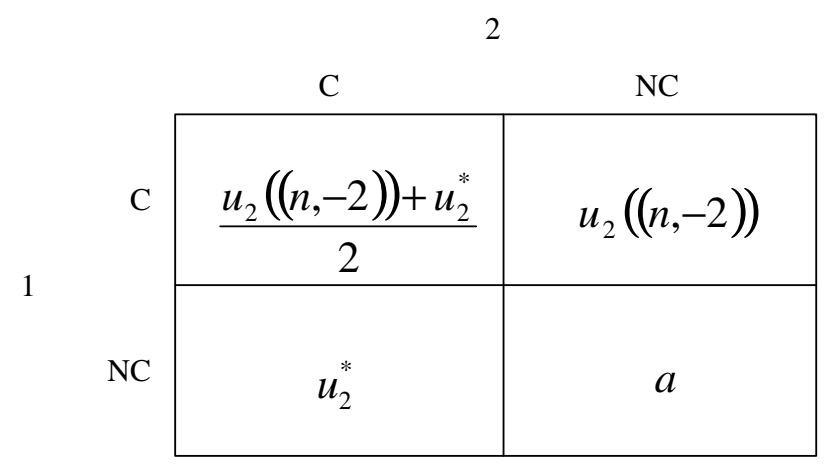

Since $u_{2}((n,-2))>u_{2}^{*}, 2$ 's indifference condition requires that $a<u_{2}^{*}$. Hence $w_{2}^{L}$, player 2's expected payoff at $\tau_{L}=(n,-2)$ conditional upon 1 not conceding and upon 2's equilibrium randomization, is strictly less than $u_{2}^{*}$.

Hence $w_{2}^{L} \leq u_{2}^{*}$. We set $w_{2}^{L}(1)=u_{2}^{*} \geq w_{2}^{L}(0) \equiv w_{2}^{L}$. 
At each stage, $P_{1}^{l+1}(1)$ solves the trivial minimization problem:

$\min x$

subject to

$$
w_{2}^{l}(0) \geq r \int_{t_{l}}^{t_{l+1}} d_{2}((s, 0)) e^{-r\left(s-t_{i}\right)} d s+e^{-r\left(t_{l+1}-t_{l}\right)}\left[w_{2}^{l+1}(1)+x\left(\tilde{\phi}_{2}\left(v_{1}^{2}\left(k^{*}(l)\right)\right)-w_{2}^{l+1}(1)\right]\right.
$$

and

$$
x \geq 0
$$

The definition of $P_{1}^{l+1}(1)$ also leads to the definition of $w_{2}^{l}(1)$ as follows:

$w_{2}^{l}(1)=r \int_{t_{l}}^{t_{l+1}} d_{2}((s, 0)) e^{-r\left(s-t_{l}\right)} d s+e^{-r\left(t_{l+1}-t_{l}\right)}\left[w_{2}^{l+1}(1)+P_{1}^{l+1}(1)\left(\tilde{\phi}_{2}\left(v_{1}^{2}\left(k^{*}(l)\right)\right)-w_{2}^{l+1}(1)\right]\right.$

Lemmas 11-13 and the assumption $\eta_{2}(\bar{\tau}(Q)) \leq \widetilde{\eta}$ imply $v_{2}^{2}\left(\tau_{L}\right) \leq \tilde{\phi}_{2}\left(v_{1}^{2}\left(k^{*}(L)\right)\right)$. Furthermore we have set $w_{2}^{L}(1)=u_{2}^{*} \geq w_{2}^{L}(0)$. Comparing equations $(9)$ and (10) and using the preceding inequalities, it may be directly verified that:

$$
P_{1}^{L}(1) \leq P_{1}^{L}(0) \text { and } w_{2}^{L-1}(1) \geq w_{2}^{L-1}(0) .
$$

Analogously, since

$$
v_{2}^{2}\left(\tau_{l+1}\right) \leq \tilde{\phi}_{2}\left(v_{1}^{2}\left(k^{*}(l+1)\right)\right), w_{2}^{l+1}(1) \geq w_{2}^{l+1}(0)
$$

it follows that at stage $(l+1)$ of the inductive definition,

$$
P_{1}^{l+1}(1) \leq P_{1}^{l+1}(0) \text { and } w_{2}^{l}(1) \geq w_{2}^{l}(0) .
$$

The next step in the argument relies on the result that $w_{2}^{l}(1) \geq u_{2}^{*}$, $l=0,1, \ldots, L-1$. To demonstrate these inequalities we first establish the following useful fact for $l=1, \ldots L-1$ : If $w_{2}^{l}(0)<u_{2}^{*}$ then $k\left(\tau_{l}\right)=-2$, $\tau_{l+1}=\left(t\left(\tau_{l}\right), k\right)$ (where $k=-1$ or +1 depending upon when player 1 has the move) and $v_{2}^{2}\left(\tau_{l+1}\right)<u_{2}^{*}$. To see this note that strictly within a round, player 1 can only reveal rationality by conceding to player 2 's current demand, which prior to $\tau^{*}$ must exceed $u_{2}^{*}$. Furthermore, strictly within a round player 2 can always obtain $u_{2}^{*}$ by conceding to player 1 . The only possibility of payoffs below $u_{2}^{*}$ arises due to 1 revealing rationality between rounds in a manner which yields normal 2 less than $u_{2}^{*}$. Player 2 accepts this eventuality precisely because of the possibility of positive probability concession by player 1 a moment earlier at the end of the preceding round 
$\left(\tau_{l}=\left(t\left(\tau_{l}\right),-2\right)\right)$ which yields player $2 u_{2}\left(\tau_{l}\right)>u_{2}^{*}$ (where $u_{2}\left(\tau_{l}\right)$ is her standing demand in the preceding round).

These considerations, and the definition of $\tau_{0}$ directly imply that $w_{2}^{0} \geq$ $u_{2}^{*}$ also. Now we argue that $w_{2}^{L-1}(1) \geq u_{2}^{*}$. Recall that $w_{2}^{L}(1)=u_{2}^{*} \geq$ $w_{2}^{L}(0) \equiv w_{2}^{L}$. Suppose, by way of contradiction, that $w_{2}^{L-1}(1)<u_{2}^{*}$. Since $w_{2}^{L-1}(1) \geq w_{2}^{L-1}(0), w_{2}^{L-1}(0)<u_{2}^{*}$ also. By the preceding discussion this is only possible if $k\left(\tau_{L-1}\right)=-2, t\left(\tau_{L}\right)=t\left(\tau_{L-1}\right)$ and $v_{2}^{2}\left(\tau_{L}\right)<u_{2}^{*}$. By Lemma $11 v_{1}^{2}\left(\tau_{L}\right)<u_{1}^{*}$, hence $\widetilde{\phi}_{2}\left(v_{1}^{2}\left(\tau_{L}\right)\right)>u_{2}^{*}$. Then the definition of $P_{1}^{L}$ yields:

$$
\begin{aligned}
P_{1}^{L}(1) & =0 \\
\text { and } w_{2}^{L-1}(1) & =0+e^{-r 0} w_{2}^{L}(1)=u_{2}^{*}
\end{aligned}
$$

which contradicts the initial supposition that $w_{2}^{L-1}(1)<u_{2}^{*}$.

Continue to suppose that the lemma is false and let

$$
l=\max \left\{m \leq L-1 \mid w_{2}^{m}(1)<u_{2}^{*}\right\} .
$$

Now we can repeat the preceding argument with $l$ replacing $L-1$ to obtain the same contradiction as before.

This demonstrates that $w_{2}^{l}(1) \geq u_{2}^{*}, \quad l=0,1, \ldots, L-1$, as required.

Define

$$
\begin{aligned}
w_{2}^{L}(2) & \equiv w_{2}^{L}(1)=u_{2}^{*} \\
w_{2}^{l}(2) & \equiv u_{2}^{*}, \quad l=1, \ldots, L-1 \\
w_{2}^{0}(2) & \equiv w_{2}^{0}(1)
\end{aligned}
$$

The $P_{1}^{l}(2)$ 's are uniquely defined by the equations:

$$
\begin{aligned}
w_{2}^{l-1}(2)= & d_{2}^{l-1}\left(1-e^{-r\left(t_{l}-t_{l-1}\right)}\right)+ \\
& +e^{-r\left(t_{L}-t_{L-1}\right)}\left[w_{2}^{l}(2)+P_{1}^{l}(2)\left(\widetilde{\phi}_{2}\left(k^{*}(l)\right)-w_{2}^{l}(2)\right)\right]
\end{aligned}
$$

where $d_{2}^{l-1}$ is the average discounted flow payoff to 2 between $t_{l-1}$ and $t_{l}$. Since $d_{2}^{l-1}<u_{2}^{*}$ (see proof of Lemma 11), the $P_{1}^{l}(2)$ so defined exist, and are strictly positive and unique.

Furthermore, we show that

$$
\begin{array}{r}
\left(1-P_{1}^{1}(1)\right)\left(1-P_{1}^{2}(1)\right) \cdots\left(1-P_{1}^{L}(1)\right) \\
\leq \quad\left(1-P_{1}^{1}(2)\right)\left(1-P_{1}^{2}(2)\right) \cdots\left(1-P_{1}^{L}(2)\right)
\end{array}
$$


as required. The $P_{1}^{2}(2)$ 's differ from the $P_{1}^{2}(1)$ 's in that the former are obtained by reducing the continuation payoffs to $u_{2}^{*}$ while keeping the initial value $w_{2}^{0}(2)$ unaltered. Much of the rest of the proof is devoted to establishing that the iterative reduction of continuation payoffs increases the compound probability that player 1 does not concede by the end of the $L$ concession episodes.

Consider

$$
\begin{aligned}
& w_{2}=\hat{d}^{1}\left(1-e^{-r \Delta_{1}}\right)+e^{-r \Delta_{1}}\left[w_{2}^{a}+x\left(\varsigma^{1}-w_{2}^{a}\right)\right] \\
& w_{2}^{b}=\hat{d}^{2}\left(1-e^{-r \Delta_{2}}\right)+e^{-r \Delta_{2}}\left[w_{2}+y\left(\varsigma^{2}-w_{2}\right)\right]
\end{aligned}
$$

where $w_{2}^{a}, w_{2}^{b}, \varsigma^{1}, \varsigma^{2}, \hat{d}^{1}$ and $\hat{d}^{2}$ are fixed, and we think of the probabilities $x$ and $y$ as functions of $w_{2}$. (Here $w_{2}^{a}$ is the continuation value $a$ fter $w_{2}$ and $w_{2}^{b}$ is the continuation value before $w_{2}$.)

Differentiating these equations with respect to $w_{2}$ yields

$$
\begin{aligned}
1 & =e^{-r \Delta_{1}}\left(\varsigma^{1}-w_{2}^{a}\right) \frac{d x}{d w_{2}} \\
0 & =\left(\varsigma^{2}-w_{2}\right) \frac{d y}{d w_{2}}+(1-y)
\end{aligned}
$$

It follows that

$$
\begin{aligned}
& \frac{d\left(1-x\left(w_{2}\right)\right)\left(1-y\left(w_{2}\right)\right)}{d w_{2}}=-(1-y) \frac{d x}{d w_{2}}-(1-x) \frac{d y}{d w_{2}}<0 \\
& \Leftrightarrow e^{r \Delta_{1}}\left(\varsigma^{2}-w_{2}\right)>(1-x)\left(\varsigma^{1}-w_{2}^{a}\right) \\
& \Leftrightarrow \varsigma^{2}-w^{2}>e^{-r \Delta_{1}} \varsigma^{1}-e^{-r \Delta_{1}} w_{2}^{a}-e^{-r \Delta_{1}} x\left(\varsigma^{1}-w_{2}^{a}\right) \\
& \Leftrightarrow \varsigma^{2}-e^{-r \Delta_{1}} \varsigma^{1}>\hat{d}^{1}\left(1-e^{-r \Delta_{1}}\right)
\end{aligned}
$$

Consequently if $\hat{d}^{1}<u_{2}^{*}$ and $\varsigma^{2} \geq \varsigma^{1}>u_{2}^{*}$, then indeed

$$
\frac{d\left(1-x\left(w_{2}\right)\right)\left(1-y\left(w_{2}\right)\right)}{d w_{2}}<0
$$


Now, if we set

$$
\begin{aligned}
w_{2}^{a} & =w_{2}^{L}(2), \Delta_{1}=t_{L}-t_{L-1} \\
\hat{d}^{1}\left(1-e^{-r \Delta_{1}}\right) & =r \int_{t_{L-1}}^{t_{L}} d_{2}(s) e^{-r\left(s-t_{L-1}\right)} d s \\
\varsigma^{1} & =\widetilde{\phi}_{2}\left(v_{1}^{2}\left(k^{*}(L)\right)\right) \\
\varsigma^{2} & =\widetilde{\phi}_{2}\left(v_{1}^{2}\left(k^{*}(L-1)\right)\right) \\
w_{2}^{b} & =w_{2}^{L-2}(1), \Delta_{2}=t_{L-1}-t_{L-2} \\
\hat{d}^{2}\left(1-e^{-r \Delta_{2}}\right) & =r \int_{t_{L-2}}^{t_{L-1}} d_{2}(s) e^{-r\left(s-t_{L-2}\right)} d s
\end{aligned}
$$

then the latter inequalities indeed hold. Observe that $w_{2}$ represents $w_{2}^{L-1}$, the $(L-1)^{t h}$ continuation value. Hence (12) implies

$$
\begin{aligned}
& \left(1-x\left(u_{2}^{*} ; L\right)\right)\left(1-y\left(u_{2}^{*} ; L\right)\right) \\
\geq & \left(1-P_{1}^{L}(1)\right)\left(1-P_{1}^{L-1}(1)\right)
\end{aligned}
$$

since

$$
\begin{aligned}
P_{1}^{L}(1) & =x\left(w_{2}^{L-1}(1)\right) \\
P_{1}^{L-1}(1) & =y\left(w_{2}^{L-1}(1)\right)
\end{aligned}
$$

and $w_{2}^{L-1}(1) \geq u_{2}^{*}$. (The argument $L$ in $x\left(u_{2}^{*} ; L\right)$ indexes the values chosen for $w_{2}^{a}, w_{2}^{b}, \varsigma^{1}, \varsigma^{2}, \hat{d}^{1}, \hat{d}^{2}$ and the time arguments in the integral.)

This step yields (only) $P_{1}^{L}(2)=x\left(u_{2}^{*} ; L\right)$. Proceeding inductively in this manner we next obtain $P_{1}^{L-1}(2)=x\left(u_{2}^{*} ; L-1\right)$, then $P_{1}^{L-2}(2)=$ $x\left(u_{2}^{*} ; L-2\right)$, and so on. For instance, the second step would entail $w_{2}^{a}=$ $w_{2}^{L-1}(2), \Delta_{1}=t_{L-1}-t_{L-2}, w_{2}^{b}=w_{2}^{L-3}(1), \varsigma^{1}=\widetilde{\phi}_{2}\left(v_{1}^{2}\left(k^{*}(L-1)\right)\right)$, $\varsigma^{2}=\widetilde{\phi}_{2}\left(v_{1}^{2}\left(k^{*}(L-1)\right)\right)$ and $w_{2}$ would represent $w_{2}^{L-2}$. It follows that

$$
\begin{aligned}
& \left(1-P_{1}^{1}(1)\right)\left(1-P_{1}^{2}(1)\right) \cdots\left(1-P_{1}^{L}(1)\right) \\
& \leq\left(1-P_{1}^{1}(1)\right)\left(1-P_{1}^{2}(1)\right) \cdots\left(1-P_{1}^{L-3}(1)\right)\left(1-y\left(u_{2}^{*} ; L\right)\right)\left(1-P_{1}^{L}(2)\right) \\
& \leq\left(1-P_{1}^{1}(1)\right)\left(1-P_{1}^{2}(1)\right) \cdots\left(1-y\left(u_{2}^{*} ; L-1\right)\right)\left(1-P_{1}^{L-1}(2)\right)\left(1-P_{1}^{L}(2)\right) \\
& \leq\left(1-P_{1}^{1}(2)\right)\left(1-P_{1}^{2}(2)\right) \cdots\left(1-P_{1}^{L}(2)\right)
\end{aligned}
$$


Finally, we set

$$
\begin{aligned}
\hat{P}_{1}^{k} & =P_{1}^{q(k)+1}(2) \\
\underline{w}_{1}^{k} & =v_{1}^{2}\left(\tau_{q(k)}\right) \\
\bar{w}_{1}^{k} & =v_{1}^{2}\left(\tau_{q(k)+1}\right) \\
\underline{t}_{k} & =t\left(\tau_{q(k)}\right) \\
\bar{t}_{k} & =t\left(\tau_{q(k)+1}\right)
\end{aligned}
$$

to obtain the desired result for a single interval. The extension to the collection of intervals is straightforward.

Lemma 16 uses the collection of up-jump intervals constructed in Lemma 15 to define modified conditional concession probabilities for 2 , to be used in the modified distribution functions of Step 7 in the text. It applies the formula for $P_{2}$ from Step 5 to those constructed intervals to get the modified probabilities for 2; this overestimates (as desired) 2's probability of concession (away from 0) because, as Lemma 16 shows, there is a partition of the actual down-jump range whose elements are subsets of the constructed intervals in question (and by the subdivision result of Step 4, every partition of that range has an aggregate implication for concession probability which weakly overestimates the actual probability of concession by 2). Lemma 15 guarantees that the modified concession probabilities it assigns to player 1 yield lower overall concession probability than the true value for 1 (as desired). Step 8 adapts the analysis for perfectly paired jumps in Step 5 to ensure that the modified up-jump probabilities (uniformly) outweigh the modified down-jump probabilities.

Lemma 16 Consider the sequence of values $\underline{w}^{y}, \bar{w}^{y}, y=1, \ldots, Y$ from the previous lemma. Define

$$
\hat{P}_{2}^{y}=\frac{\bar{w}_{1}^{y}-\underline{w}_{1}^{y}}{u_{1}^{*}-\underline{w}_{1}^{y}} \quad y=1, \ldots, Y
$$

and

$$
P_{2}=\frac{a-b}{u_{1}^{*}-b}
$$

Then

$$
\left(1-P_{2}\right) \geq\left(1-\hat{P}_{2}^{1}\right) \ldots\left(1-\hat{P}_{2}^{Y}\right)
$$

Proof. Consider the sequence of values as defined in Lemma 15, and construct the following new sequences $\underline{v}_{1}^{y}, \bar{v}_{1}^{y}, y=1, \ldots, Y$ where $\underline{v}_{1}^{y}=\bar{w}^{y-1}$, $\bar{v}_{1}^{y}=\min \left\{a, \bar{w}^{y}\right\}$, and we define $\bar{w}^{0}=b$. 
The intervals $\left[\underline{v}_{1}^{y}, \bar{v}_{1}^{y}\right]$ partition $[b, a]$. Down jumps over the range $[b, a]$ may be subdivided (see Step 4) into $Y$ down jumps from $\underline{v}_{1}^{y}$ to $\bar{v}_{1}^{y}, y=$ $1, \ldots, Y$ respectively. Let $\tilde{P}_{2}^{y}$ denote the positive probability of concession by 2 associated with a down jump from $\underline{v}_{1}^{y}$ to $\bar{v}_{1}^{y}$. Then $\left(1-\tilde{P}_{2}^{y}\right) \leq \frac{u_{1}^{*}-\bar{v}_{1}^{y}}{u_{1}^{*}-\underline{v}_{1}^{y}}$ by Lemma 9 . Let $\hat{P}_{2}^{y}$ be defined by $\left(1-\hat{P}_{2}^{y}\right)=\frac{u_{1}^{*}-\bar{w}_{1}^{y}}{u_{1}^{*}-\underline{w}_{1}^{y}}$, that is, correspond to a down jump from $\bar{w}_{1}^{y}$ to $\underline{w}_{1}^{y}$. Then clearly $\left(1-\tilde{P}_{2}^{y}\right) \geq\left(1-\hat{P}_{2}^{y}\right)$. Consequently, $\left(1-P_{2}\right)=\left(1-\tilde{P}_{2}^{1}\right) \ldots\left(1-\tilde{P}_{2}^{Y}\right) \geq\left(1-\hat{P}_{2}^{1}\right) \ldots\left(1-\hat{P}_{2}^{Y}\right)$

\section{Section 5: Existence}

In the notation defined in Section 5 , let $\mathcal{C}\left(z, \Delta_{k}, \bar{\tau}\right), k=1,2, \ldots$ be a sequence of $\bar{\tau}$-truncated, discrete-time concession games such that $\Delta_{k} \in$ $(0,1)$ decreases monotonically to zero and $t(\bar{\tau})<\infty$.

An equilibrium of $\mathcal{C}\left(z, \Delta_{k}, \bar{\tau}\right)$ is specified by the behavior of the normal types of both players $\left(\mu_{i}^{k} ; H_{i}^{k}(\cdot \mid \gamma), \gamma \in \Gamma\right)_{i=1,2}$, where $\mu_{i}^{k}\left(\gamma_{i}\right)$ is the probability with which a normal type of player $i$ mimics $\gamma_{i} \in \Gamma_{i}$ and $H_{i}^{k}\left(\tau \mid \gamma_{1}, \gamma_{2}\right)$ is the probability with which normal $i$ concedes to $j$ by date $\tau$ (inclusive) in the game following the choice of types $\left(\gamma_{1}, \gamma_{2}\right) \in \Gamma_{1} \times \Gamma_{2}$.

Let $h_{i}^{k}(\tau \mid \gamma)$ denote the ex-ante probability that a normal player $i$ concedes/reveals rationality at $\tau$, given $\gamma_{1}, \gamma_{2}$. Then

$$
H_{i}^{k}(\tau \mid \gamma)=\sum_{\tau^{\prime} \preccurlyeq \tau} h_{i}^{k}\left(\tau^{\prime} \mid \gamma\right)
$$

Since $\mathcal{C}\left(z, \Delta_{k}, \bar{\tau}\right)$ is a finite extensive form game, an equilibrium exists. Let $\bar{T}=t(\bar{\tau})$ and define $\tilde{H}_{i}^{k}:[-1, \bar{T}] \rightarrow[0,1]$ as

$$
\tilde{H}_{i}^{k}(s)=\left\{\begin{array}{cc}
0 & \text { if } s \in[-1,0) \\
H_{i}^{k}((s, 0)) & \text { if } s>0, s \notin \mathcal{N} \\
H_{i}^{k}((s,+2)) & \text { if } s \in \mathcal{N}
\end{array}\right.
$$

and $\tilde{h}_{i}^{k}:[-1, \bar{T}] \rightarrow[0,1]$ as

$$
\tilde{h}_{i}^{k}(s)=\sum_{\tau: t(\tau)=s} h_{i}^{k}(\tau)
$$

Lemma 17 There exists $\bar{\tau}$ with $t(\bar{\tau})<\infty$ such that for all $\Delta \in[0,1]$ (where $\Delta=0$ corresponds to the continuous time concession game) and in any perfect Bayesian equilibrium of $\mathcal{C}(z, \Delta)$, and after any choice of postures 
$\left(\gamma_{1}, \gamma_{2}\right)$, normal player $i$ concedes to player $j$ with probability 1 by date $\bar{\tau}$, conditional upon the game not having terminated prior to $\bar{\tau}$.

Proof. If the postures $\left(\gamma_{1}, \gamma_{2}\right)$ lead to more than compatible demands at some first date $(n,+1)$, the result follows trivially. If not, the argument is essentially the same as in Step 1 of the proof of Lemma 1.

Henceforth we take $\bar{\tau}$ to be such that Lemma 17 applies. Consequently a normal player must, in equilibrium, concede with probability 1 by date $\bar{\tau}$. Hence, $\tilde{H}_{i}^{k}$ is a distribution function (if $H_{i}^{k}$ corresponds to an equilibrium). Note that we have defined the $\tilde{H}_{i}^{k}$ 's over the domain $[-1, \bar{T}]$ (as opposed to simply $[0, \bar{T}])$ to clarify that the "tightness" condition referred to below is satisfied.

Let $\mathcal{N}^{+}(\bar{\tau})=\{\tau \mid t(\tau) \in \mathcal{N}$ and $\tau \preccurlyeq \bar{\tau}\}$. Since the set of dates in $\mathcal{N}^{+}(\bar{\tau})$ and the set of types for each $i$ are finite, there exists a subsub...subsequence (which, abusing notation we also denote by $k$ ) for which $\mu_{i}^{k}\left(\gamma_{i}\right)$ and $H_{i}^{k}(\tau \mid \gamma)$ converge for all $\gamma_{i} \in \Gamma_{i}, \tau \in \mathcal{N}^{+}(\bar{\tau})$ and $i=1,2$.

We now obtain convergence for all $\tau$ strictly within rounds, that is,

$$
\tau \in((n,+1),(n+1,-1))
$$

First note that by Helly's theorem (Theorem 25.9) and Theorem 25.10 (the tightness condition of the latter applies trivially), both from Billingsley(1986), there exists a subsub...subsequence (which again we index by $k$ ) such that $\forall i, \forall \gamma \in \Gamma$ there exists a distribution function $\tilde{H}_{i}(\cdot \mid \gamma):[-1, \bar{T}] \rightarrow$ $[0,1]$ such that $\tilde{H}_{i}^{k}(\cdot \mid \gamma):[-1, \bar{T}] \rightarrow[0,1]$ converges to $\tilde{H}_{i}(\cdot \mid \gamma)$ at every continuity point of $\tilde{H}_{i}(\cdot \mid \gamma)$.

Lemma 18 Consider $\mathcal{C}\left(z, \Delta_{k}, \bar{\tau}\right)$ and an associated equilibrium $\left(\mu_{i}^{k}, H_{i}^{k}\right)_{i=1,2}$. Fix $\gamma \in \Gamma$ and consider $t \in(n, n+1)$ such that player 1 moves at time $t$ and $t \geq n+3 \Delta_{k}$. If $\tilde{h}_{1}^{k}(t \mid \gamma)>0$ then $\tilde{h}_{2}^{k}\left(t-\Delta_{k} \mid \gamma\right), \tilde{h}_{1}^{k}(t-$ $\left.2 \Delta_{k} \mid \gamma\right), \ldots, \tilde{h}_{2}^{k}\left(n+2 \Delta_{k} \mid \gamma\right)$ are all strictly positive. Similarly if $\tilde{h}_{2}^{k}(t \mid \gamma)>0$, for $t \geq n+4 \Delta_{k}$, then $\tilde{h}_{1}^{k}\left(t-\Delta_{k} \mid \gamma\right), \tilde{h}_{2}^{k}\left(t-2 \Delta_{k} \mid \gamma\right), \ldots, \tilde{h}_{2}^{k}\left(n+3 \Delta_{k} \mid \gamma\right)$ are all strictly positive.

Proof. This follows from the standard war of attrition logic (that is, the only reason for a player to delay conceding is the possibility that the opponent will concede in the interim) applied to the discrete-time alternating-move case. When $t=n+2 \Delta_{k}$, it is possible that $\tilde{h}_{1}^{k}\left(n+\Delta_{k} \mid \gamma\right)=0$, since $n+2 \Delta_{k}$ is the first date after $(n,+1)$ at which 2 has an opportunity to move, and 2 might delay conceding because $\tilde{h}_{1}^{k}(n \mid \gamma)>0$ (specifically, $\left.h_{1}^{k}((n,+1) \mid \gamma)>0\right)$.

Lemma $19 \tilde{H}_{i}(t \mid \gamma)$ is continuous at all $t \notin \mathcal{N}$.

Proof. Suppose not and that for some $n$ with $t \in(n, n+1), \tilde{H}_{i}(t \mid \gamma)$ has an upward jump at $t$ of size $2 a>0$. For any $\varepsilon^{\prime}>0$ there exists $\varepsilon \in\left[0, \varepsilon^{\prime}\right]$ such 
that $t-\varepsilon$ and $t+\varepsilon$ are continuity points of $\tilde{H}_{i}$. Hence, by Helly's theorem, $\lim _{k \uparrow \infty}\left[\tilde{H}_{i}^{k}(t+\varepsilon \mid \gamma)-\tilde{H}_{i}^{k}(t-\varepsilon \mid \gamma)\right] \geq 2 a$.

Hence, there exists $\frac{i}{k}<\infty$ such that for all $k \geq \bar{k}, \tilde{H}_{i}^{k}(t+\varepsilon \mid \gamma)-\tilde{H}_{i}^{k}(t-$ $\varepsilon \mid \gamma) \geq a$. Consequently for small enough $\varepsilon^{\prime}>0$, player $j$ should not concede between $(t-2 \varepsilon)$ and $(t-\varepsilon)$ (that is, immediately prior to $(t-\varepsilon)$ ), generating a contradiction with Lemma 18 for $k \geq \bar{k}$ such that $\Delta_{k}<(t-n-2 \varepsilon) / 4$.

It follows from Lemma 19 and the cited theorems from Billingsley(1986), that $H_{i}^{k}(\tau \mid \gamma)$ converges for all $\tau$ such that $t(\tau) \notin N$. We have chosen a subsequence such that $H_{i}^{k}(\tau \mid \gamma)$ (and $\mu_{i}^{k}\left(\gamma_{i}\right)$ ) converges for $i \in\{1,2\}$, all $\gamma \in \Gamma$ and $\tau \in \mathcal{N}^{+}(\bar{\tau})$. Hence $\lim _{k} H_{i}^{k}(\tau \mid \gamma)$ exists for all $\tau \preccurlyeq \bar{\tau}$.

Let $H_{i}(\tau \mid \gamma) \equiv \lim _{k} H_{i}^{k}(\tau \mid \gamma)$. Define $h_{i}(\tau \mid \gamma) \equiv H_{i}(\tau \mid \gamma)-\lim _{\tau^{\prime} \uparrow \tau} H_{i}\left(\tau^{\prime} \mid \gamma\right)$.

Let $\mu_{i}\left(\gamma_{i}\right) \equiv \lim _{k} \mu_{i}^{k}\left(\gamma_{i}\right)$. We will argue that $\left(\mu_{i}, H_{i}\right)_{i=1,2}$ is an equilibrium of $\mathcal{C}(z, \bar{\tau})$.

We say that $\tau$ is a point of increase of $H_{i}(. \mid \gamma)$ if $h_{i}(\tau \mid \gamma)>0$ or if for all $\tau^{\prime}, \tau^{\prime \prime}$ such that $\tau^{\prime} \prec \tau \prec \tau^{\prime \prime}, H_{i}\left(\tau^{\prime} \mid \gamma\right)<H_{i}(\tau \mid \gamma)<H_{i}\left(\tau^{\prime \prime} \mid \gamma\right)$. If $\tau \in \mathcal{N}^{+}$and $\tau$ is a point of increase of $H_{i}(. \mid \gamma)$, then it must be the case that $h_{i}(\tau \mid \gamma)>0$.

Applying the above definition to $H_{i}^{k}(. \mid \gamma)$, observe that $\tau$ is a point of increase of $H_{i}^{k}(. \mid \gamma)$ if and only if $h_{i}^{k}(\tau \mid \gamma)>0$.

Let $U_{i}\left(\tau \mid \gamma_{i}, \gamma_{j}\right)$ be the expected payoff to player $i$ of conceding/revealing rationality at $\tau$ given $\left(\mu_{l}, H_{l}\right)_{l=1,2}$ and conditional upon $\left(\gamma_{i}, \gamma_{j}\right)$ being chosen at the start of play. A pure strategy for player $i$ is a choice of some $\gamma_{i} \in \Gamma_{i}$ and a set of dates $\left\{\tau_{\gamma_{j}} \mid \gamma_{j} \in \Gamma_{j}\right\}$ such that $i$ concedes at $\tau_{\gamma_{j}}$ if $j$ chooses $\gamma_{j}$ at the start of play. It follows that the tuple $\left(\mu_{i}, H_{i}\right)_{i=1,2}$ is an equilibrium of $(\mathcal{C}, \Delta, \bar{\tau})$ if for all $\gamma_{i} \in \Gamma_{i}$ such that $\mu_{i}\left(\gamma_{i}\right)>0$, and for any set of dates $\left\{\tau_{\gamma_{j}} \mid \gamma_{j} \in \Gamma_{j}\right\}$ such that for each $\gamma_{j}, \tau_{\gamma_{j}}$ is a point of increase of $H_{i}\left(\cdot \mid \gamma_{i}, \gamma_{j}\right)$,

$$
\begin{aligned}
& \sum_{\gamma_{j}} U_{i}\left(\tau_{\gamma_{j}} \mid \gamma_{i}, \gamma_{j}\right)\left[z_{j} \pi_{i}\left(\gamma_{j}\right)+\left(1-z_{j}\right) \mu_{i}\left(\gamma_{j}\right)\right] \\
\geq & \sum_{\gamma_{j}} U_{i}\left(\tau_{\gamma_{j}}^{\prime} \mid \gamma_{i}^{\prime}, \gamma_{j}\right)\left[z_{j} \pi_{i}\left(\gamma_{j}\right)+\left(1-z_{j}\right) \mu_{i}\left(\gamma_{j}\right)\right]
\end{aligned}
$$

$$
\text { for all } \gamma_{i}^{\prime} \in \Gamma_{i} \text { and }\left\{\tau_{\gamma_{j}}^{\prime} \mid \gamma_{j} \in \Gamma_{j}\right\} \text {. }
$$

This corresponds to the usual definition, according to which pure strategies used in equilibrium must yield at least as high a payoff as any other pure strategies.

Let $\mathcal{T}_{i}^{k}$ be the set of dates at which player $i$ moves in the concession game $\mathcal{C}\left(z, \Delta_{k}, \bar{\tau}\right)$ and define

$$
\zeta_{i}^{k}(\tau)=\min \left\{\tau^{\prime} \in \mathcal{T}_{i}^{k} \mid \tau^{\prime} \succcurlyeq \tau\right\}
$$


Lemma 20 If $\tau$ is a point of increase of $H_{i}(. \mid \gamma)$ then there exists $\bar{k}$ such that $h_{i}^{k}\left(\zeta_{i}^{k}(\tau) \mid \gamma\right)>0$ for $k \geq \bar{k}$.

Proof. If $\tau \in \mathcal{N}^{+}(\bar{\tau})$, then since $\tau$ is a point of increase of $H_{i}(. \mid \gamma)$, $h_{i}(\tau \mid \gamma)>0$ and $\zeta_{i}^{k}(\tau)=\tau$. We have chosen a sequence such that $h_{i}^{k}(\tau \mid \gamma) \rightarrow$ $h_{i}(\tau \mid \gamma)$ for all $\tau \in \mathcal{N}^{+}(\bar{\tau})$. The conclusion now follows directly in this case. Now suppose $\tau \notin \mathcal{N}^{+}$. Then $t(\tau) \in(n, n+1)$ for some $n$ and Lemma 18 applies. Let $\bar{\tau}_{k}=\max \left\{\tau^{\prime} \mid t\left(\tau^{\prime}\right) \in(n, n+1)\right.$ and $\left.h_{i}^{k}\left(\tau^{\prime} \mid \gamma\right)>0\right\}$. Then $\bar{\tau}_{k} \succ \tau$ for $k$ large enough. If not, there exists a subsequence $\left(k_{l}\right)$ with $\bar{\tau}_{k_{l}} \preceq \tau$ along the subsequence. It follows from Lemma 18 that $H_{i}^{k_{l}}\left(\tau^{\prime \prime} \mid \gamma\right)=H_{i}^{k_{l}}(\tau \mid \gamma)$ for all $\tau^{\prime \prime} \succ \tau$ such that $t\left(\tau^{\prime \prime}\right)<(n+1)$.

Consequently $H_{i}\left(\tau^{\prime \prime} \mid \gamma\right)=\lim _{l \rightarrow \infty} H_{i}^{k_{l}}\left(\tau^{\prime \prime} \mid \gamma\right)=\lim _{l \rightarrow \infty} H_{i}^{k_{l}}(\tau \mid \gamma)=$ $H_{i}(\tau \mid \gamma)$, contradicting the initial assumption that $\tau$ is a point of increase of $H_{i}(. \mid \gamma)$.

It follows from the preceding lemma that

$$
\begin{aligned}
& \sum_{\gamma_{j}} U_{i}^{k}\left(\zeta_{i}^{k}\left(\tau_{\gamma_{j}}\right) \mid \gamma_{i}, \gamma_{j}\right)\left[z_{j} \pi_{j}\left(\gamma_{j}\right)+\left(1-z_{j}\right) \mu_{j}^{k}\left(\gamma_{j}\right)\right] \\
\geq & \sum_{\gamma_{j}} U_{i}^{k}\left(\zeta_{i}^{k}\left(\tau_{\gamma_{j}}^{\prime}\right) \mid \gamma_{i}^{\prime}, \gamma_{j}\right)\left[z_{j} \pi_{j}\left(\gamma_{j}\right)+\left(1-z_{j}\right) \mu_{j}^{k}\left(\gamma_{j}\right)\right]
\end{aligned}
$$

for all $\gamma_{i}^{\prime} \in \Gamma_{i}$ and $\left\{\tau_{\gamma_{j}}^{\prime} \mid \gamma_{j} \in \Gamma_{j}\right\}$.

Lemma 21 For $i=1,2$ and all $\left(\gamma_{1}, \gamma_{2}\right) \in \Gamma_{1} \times \Gamma_{2}$ and $\tau \preccurlyeq \bar{\tau}$,

$$
U_{i}^{k}\left(\zeta_{i}^{k}(\tau) \mid \gamma_{1}, \gamma_{2}\right) \rightarrow U_{i}\left(\tau \mid \gamma_{1}, \gamma_{2}\right)
$$

Proof. Fix $\left(\gamma_{1}, \gamma_{2}\right)$ and $i$, and for notational simplicity suppress the arguments $\left(\gamma_{1}, \gamma_{2}\right)$ in the various functions below. Let $V_{i}(\tau)$ be the realized payoff to $i$ if player $j$ concedes at $\tau$ and $i$ does not concede at or before $\tau$. Then

$$
V_{i}(\tau)=\int_{0}^{t(\tau)} d_{i}((s, 0)) e^{-r s} d s+e^{-r t(\tau)} u_{i}(\tau) .
$$

Let

$$
\hat{H}_{j}^{k}(s)=\left\{\begin{array}{cc}
\tilde{H}_{j}^{k}(s) & \text { for } s \notin \mathcal{N} \\
\tilde{H}_{j}^{k}(n)-h_{j}^{k}((n,+2)) & \text { for } n \in \mathcal{N}
\end{array}\right.
$$

and

$$
\hat{V}_{i}(s)=\left\{\begin{array}{cc}
V_{i}((s, 0)) & \text { for } s \notin \mathcal{N} \\
V_{i}((n,-2)) & \text { for } n \in \mathcal{N}
\end{array}\right.
$$


For $x \in \mathbb{R}$ let $\lfloor x\rfloor$ denote the largest integer less than or equal to $x$. Then

$$
\begin{aligned}
U_{i}^{k}\left(\zeta_{i}^{k}(\tau) \mid \gamma_{1}, \gamma_{2}\right)= & \sum_{n=0}^{\lfloor t(\tau)\rfloor} h_{j}^{k}((n,+2)) V_{i}((n,+2))+ \\
& +\sum_{n=0}^{\lfloor t(\tau)\rfloor-1} \int_{n}^{\min \left\{n+1, t\left(\zeta_{i}^{k}(\tau)\right)\right\}} \hat{V}_{i}(s) d \hat{H}_{j}^{k}(s)-h_{j}^{k}\left(\zeta_{i}^{k}(\tau)\right) V_{i}(\tau)+ \\
& +h_{j}^{k}\left(\zeta_{i}^{k}(\tau)\right)\left[\int_{0}^{t\left(\zeta_{i}^{k}(\tau)\right)} d_{i}((s, 0)) e^{-r s} d s+\right. \\
& \left.+e^{-r t\left(\zeta_{i}^{k}(\tau)\right)} \frac{1}{2}\left[u_{i}\left(\zeta_{i}^{k}(\tau)\right)+\phi_{i}\left(u_{j}\left(\zeta_{i}^{k}(\tau)\right)\right)\right]\right]+ \\
& +\left(1-H_{j}^{k}\left(\zeta_{i}^{k}(\tau)\right)\right)\left[\int_{0}^{t\left(\zeta_{i}^{k}(\tau)\right)} d_{i}((s, 0)) e^{-r s} d s+\right. \\
& \left.+e^{-r t(\tau)} \phi_{i}\left(u_{j}\left(\zeta_{i}^{k}(\tau)\right)\right)\right]
\end{aligned}
$$

The term $U_{i}\left(\tau \mid \gamma_{1}, \gamma_{2}\right)$ has the same form except that the $k$ superscripts are missing. In the expression above, because of the alternating move structure, $h_{j}^{k}\left(\zeta_{i}^{k}(\tau)\right)=0$ unless $\tau \in \mathcal{N}^{+}$. In this case, of course, $\zeta_{i}^{k}(\tau)=\tau$. For $\tau \in \mathcal{N}^{+}$it follows by the construction of our initial sub...subsequence that $h_{j}^{k}\left(\zeta_{i}^{k}(\tau)\right) \rightarrow h_{j}\left(\zeta_{i}^{k}(\tau)\right)$.

We complete the proof by establishing that

$$
\int_{n}^{m} \hat{V}_{i}(s) d \hat{H}_{j}^{k}(s) \rightarrow \int_{n}^{m} \hat{V}_{i}(s) d \hat{H}_{j}(s)
$$

where $m=\min \left\{n+1, t\left(\zeta_{i}^{k}(\tau)\right)\right\}$.

Integrating by parts yields:

$$
\left.\int_{n}^{m} \hat{V}_{i}(s) d \hat{H}_{j}^{k}(s)=\hat{V}_{i}(s) \hat{H}_{j}^{k}(s)\right]_{n}^{m}-\int_{n}^{m} \hat{H}_{j}^{k}(s) d \hat{V}_{i}(s)
$$

The first term on the right hand side clearly converges to $\left.\hat{V}_{i}(s) \hat{H}_{j}(s)\right]_{n}^{m}$.

Now consider the second term:

$$
\int_{n}^{m} \hat{H}_{j}^{k}(s) d \hat{V}_{i}(s)=\int_{n}^{m} \hat{H}_{j}^{k}(s)\left[d_{i}((n,+2))-r u_{i}((n,+2))\right] e^{-r s} d s
$$

since $\hat{V}_{i}^{\prime}(s)=\left[d_{i}((n,+2))-r u_{i}((n,+2))\right]$. 
Furthermore $\hat{H}_{j}^{k}(s) \rightarrow \tilde{H}_{j}(s)$ for all $s \notin \mathcal{N}$. The desired conclusion now follows directly from the Lebesgue Convergence Theorem (see Theorem 15, Chapter 4, Royden(1968)).

Lemma 22 For all $\Delta \in[0,1]$, a Nash equilibrium of $\mathcal{C}(z, \Delta, \bar{\tau})$ is also a Nash equilibrium of $\mathcal{C}(z, \Delta)$.

Proof. By Lemma 17, following the choice of $\gamma_{i}$ by a normal player $i$, there is no strategy of player $j$ for which it is a best response for normal $i$ to concede after date $\bar{\tau}$ (conditional upon player $j$ not having conceded to $i$ prior to that time). The result follows directly.

Lemma 23 The concession game $\mathcal{C}(z)$ has a Nash equilibrium.

Proof. To establish the lemma, take limits w.r.t. $k$ in (14). By Lemma 21, taking limits yields (13), establishing that $\left(\mu_{i}, H_{i}\right)_{i=1,2}$ defines an equilibrium of $C(z, \bar{\tau})$. By Lemma 17, this is also a Nash equilibrium of $\mathcal{C}(z)$. 


\section{References}

[1] Abreu, D. and F. Gul (2000), "Bargaining and Reputation," Econometrica, 68: 85- 117.

[2] Abreu, D. and D. Pearce (2002), "Bargaining, Reputation and Equilibrium Selection in Repeated Games," mimeo.

[3] Aoyagi, M. (1996), "Reputation and Dynamic Stackelberg Leadership in Infinitely Repeated Games," Journal of Economic Theory, 71: 3.

[4] Aumann, R. and S. Sorin (1989), "Cooperation and Bounded Recall," Games and Economic Behavior, 1: 5-39

[5] Billingsley, P. (1986), "Probability and Measure," 2nd edition, John Wiley and Sons.

[6] Bulow, J. (1982), "Durable Goods Monopolists," Journal of Political Economy, 90: 314-322.

[7] Busch, L-A and Q. Wen (1995), "Perfect Equilibria in a Negotiation Model," Econometrica, 63 (3): 545-565.

[8] Celentani, M., D. Fudenberg, D. Levine, and W. Pesendorfer (1996), "Maintaining a Reputation Against a Long-Lived Opponent," Journal of Economic Theory, 64: 691-704.

[9] Chan, J. (2000), "On the Non-existence of Reputational Effects in Two-Person Infinitely-Repeated Games," working paper, Department of Economics, Johns Hopkins University.

[10] Coase, R. (1972), "Durability and Monopoly," Journal of Law and Economics, 15: 143-149.

[11] Compte, O. and P. Jehiel (2002), "On the Role of Outside Options in Bargaining with Obstinate Parties," Econometrica, 70: 1477-1517.

[12] Cripps, M., E. Dekel and W. Pesendorfer (2005), "Reputation with Equal Discounting in Repeated Games with Strictly Conflicting Interests," Journal of Economic Theory, 121: 259-272.

[13] Cripps, M., K. Schmidt and J.P. Thomas (1996), "Reputation in Perturbed Repeated Games," Journal of Economic Theory, 69: 387-410. 
[14] Cripps, M. and J. Thomas (1997), "Reputation and Perfection in Repeated Common Interest Games," Games and Economic Behavior, 18: $141-158$.

[15] Fernandez, R. and J. Glazer (1991), "Striking for a Bargain between Two Completely Informed Agents," American Economic Review, 81: $240-252$.

[16] Fudenberg, D. and D. Levine (1989), "Reputation and Equilibrium Selection in Games with a Patient Player," Econometrica, 57: 759-778.

[17] Fudenberg, D., D. Levine and J. Tirole (1985), "Infinite-Horizon Models of Bargaining with One-Sided Incomplete Information," In GameTheoretic Models of Bargaining, ed. A. Roth, Cambridge University Press.

[18] Gul, F., H. Sonnenschein, and R. Wilson (1986), "Foundations of Dynamic Monopoly and the Coase Conjecture," Journal of Economic Theory, 39: $155-190$.

[19] Haller, H. and S. Holden (1990), "A Letter to the Editor on Wage Bargaining", Journal of Economic Theory, 52: 232-236.

[20] Kambe, S. (1999), "Bargaining with Imperfect Commitment," Games and Economic Behavior, 28: 217-237.

[21] Kreps, D., P. Milgrom, J. Roberts and R. Wilson (1982), "Rational Cooperation in the Finitely Repeated Prisioners' Dilemma," Journal of Economic Theory, 27: 245-252.

[22] Kreps, D. and R. Wilson (1982), "Reputation and Imperfect Information," Journal of Economic Theory, 27: 253-279.

[23] Kornhauser, L., A. Rubinstein and C.Wilson (1989), "Reputation and Patience in the War of Attrition," Economica 56, 15-24.

[24] Milgrom, P. and J. Roberts (1982), "Predation, Reputation and Entry Deterrence," Journal of Economic Theory, 27: 280-312.

[25] Myerson, R. (1991), "Game Theory: Analysis of Conflict," Cambridge, Massachusetts, Harvard University Press.

[26] Nash, J. (1950a), "The Bargaining Problem," Econometrica, 18: 155162. 
[27] Nash, J. (1950b), "Equilibrium Points in n-person Games," Proceedings of the National Academy of Sciences, 36: 48-49.

[28] Nash, J. (1953), "Two-Person Cooperative Games," Econometrica, 21: $128-140$.

[29] Pearce, D. (1989), "Renegotiation-Proof Equilibria: Collective Rationality and Intertemporal Cooperation," Cowles Foundation Discussion Paper 855, Yale University.

[30] Royden, H. L. (1968), "Real Analysis," 2nd edition, The Macmillan Company.

[31] Rubinstein, A. (1982), "Perfect Equilibrium in a Bargaining Model," Econometrica, 50: 97-109.

[32] Schmidt, K. (1993), "Reputation and Equilibrium Characterization in Repeated Games of Conflicting Interests," Econometrica, 61: 325-351.

[33] Smith, L. and E. Stacchetti (2001), "Aspirational Bargaining," University of Michigan working paper.

[34] Stokey, N. (1981), "Rational Expectations and Durable Goods Pricing," Bell Journal of Economics, 12: 112-128. 